\title{
CYLINDRICAL TANGENT CONES AND THE SINGULAR SET OF MINIMAL SUBMANIFOLDS
}

\author{
LEON SIMON
}

The question of what can be said about the structure of the singular set of minimal surfaces and the extrema of other geometric variational problems has remained largely open. Indeed, for minimal surfaces, apart from various upper bounds on the possible dimension of the singular set (see, e.g., [1], [5], [7], [8], [10], [13], [17], [19]), little has been known beyond the work of Jean Taylor [22], [23] and Brian White [24], [25] concerning $\bmod p$ and $"(M, \varepsilon, \delta)$ " minimizing hypersurfaces, where the tangent cones are of very special (and unvarying) type, and there are topological obstructions to perturbing away the singularities.

Here we prove rectifiability and local finiteness of measure of the singular set for various classes of minimal submanifolds, including for the first time cases where the tangent cones may have varying type and where there is no topological obstruction to perturbing away the singularities. For example we establish here (in Corollary 1 of $\S 1)$ the $(n-2)$-rectifiability for the interior singular set of any mod 2 minimizing current of arbitrary codimension, and local finiteness for the $(n-2)$-dimensional measure of the "top-dimensional" part of this singular set. Perhaps more importantly, the work here produces some analytic machinery which seems to hold promise for further developments.

The key result of the present work is a technical decay lemma, Lemma 1 of $\S 1$. This lemma says roughly that, if $M \subset \mathbf{R}^{n+k}$ lies in a suitable "multiplicity one class" $\mathscr{M}$ of $n$-dimensional minimal submanifolds $\subset$ $\mathbf{R}^{n+k}$ (described precisely in $\S 1$ ), and if $M$ is close to $\mathrm{C}$ in a ball $B_{\rho}$ in a suitable $L^{2}$ sense (made precise in Lemma 1 ), where $\mathbf{C}=\mathbf{C}_{0} \times \mathbf{R}^{m}$ is a cylindrical cone having cross section $\mathbf{C}_{0}$ satisfying an "integrability condition" (see $\ddagger \ddagger$ of $\S 1$ ), then either there is a significant "gap" in the part of the singular set consisting of points $X \in B_{\rho}$ where the density $\Theta_{m}(X)$ of $M$ at $X \geq$ the density $\Theta_{\mathbf{C}}(0)$ of $\mathbf{C}$ at 0 , or else there is a cylindrical

Received September 16, 1991. Research partly supported by NSF grant DMS-9012718 at Stanford University. 
cone $\widetilde{\mathbf{C}}$ close to $\mathbf{C}$ such that the quantity $\rho^{-n-2} \int_{M \cap B_{\rho}} \operatorname{dist}^{2}(X, \widetilde{\mathbf{C}})$ decays by a fixed factor as we reduce radius by a fixed factor.

While methods involving "improvement of excess" as in the second alternative above are by now quite standard in regularity theory (see, e.g., [1], [2], [5], [6], [8], [13], [16], [17]), a result like Lemma 1 (which says that either we get excess improvement or else there is a significant gap in the singular set) has not, as far as we are aware, been previously utilized in the study of geometric extrema. It seems likely that the same kind of result could be usefully applied in various other contexts-certainly all the main results here have analogues in the study of energy minimizing maps between Riemannian manifolds $M, N$; these will be described in detail in [21].

A direct consequence of Lemma 1 is the decomposition theorem (Theorem 1 of $\S 1)$ which says that, for suitable $\delta \in(0,1)$, under hypotheses similar to those of Lemma 1, except that $M$ should be suitably close to $C$ in $B_{2 \rho}$, we get a decomposition of the form

$$
\left\{X \in B_{\rho}: \Theta_{M}(X) \geq \Theta_{\mathbf{C}}(0)\right\}=S \cup T,
$$

$S \subset L, L$ an $m$-dimensional embedded $C^{1, \alpha}$ manifold with $\operatorname{vol}(L) \leq$ $\omega_{m} \rho^{m}$, and $T \subset \bigcup_{j} B_{\rho_{j}}\left(X_{j}\right)$ for some family $B_{\rho_{j}}\left(X_{j}\right)$ of balls with $\sum_{j=1}^{N} \rho_{j}^{m} \leq(1-\delta) \rho^{m}$. The nature of this result suggests the possibility of repeated iteration, starting at the second stage with a suitable scaling and translation of $M \cap B_{\rho_{j}}\left(X_{j}\right)$ in place of $M \cap B_{\rho}$. Such an iteration is indeed possible under the appropriate circumstances (the main point is that one must be able to check the starting hypotheses at each new stage), and the main theorem about the structure of the singular set (Theorem 2 of $\S 1$ ) is obtained in exactly this way.

Corollary 1 of Theorem 2 settles a well-known question about the rectifiability of the singular set of mod 2 minimizing currents of arbitrary dimension and codimension, and also establishes a local finiteness result for the $(n-2)$-dimensional measure of the singular set.

A second class of results is obtained when we are in a setting which makes it possible to check that the first alternative of the technical lemma above (i.e., the alternative that there can be a significant "gap" in the appropriate part of the singular set) can be ruled out a-priori. This class of results is given in Theorem 4 of $\S 1$ and its corollaries. For example Corollary 2 shows that the results in [22], [23] and [24], [25] relating to the "top-dimensional part" of the singular set can be generalized to a stationary setting in arbitrary codimension. In Corollary 3 we also prove that if 
$X_{0}$ is a singular point of density less than 2 for any $n$-dimensional integral varifold $\mathbf{V}$ which is stationary in an open subset $U \subset \mathbf{R}^{n+k} \quad(k \geq 1$ arbitrary), then there is $\rho>0$ such that the singular set of $\mathbf{V}$ in $B_{\rho}\left(X_{0}\right)$ is the union of an embedded $C^{1, \alpha}$ manifold and a set of dimension $\leq n-2$. If $n=2$, then we prove the more precise result that $\operatorname{sing} \mathbf{V}$ is either a properly embedded $C^{1, \alpha}$ Jordan arc, or else is a finite union of properly embedded locally $C^{1, \alpha}$ Jordan arcs of finite length, each with one endpoint at $X_{0}$ and one endpoint in $\partial B_{\rho}\left(X_{0}\right)$. Of course all the above results extend naturally to multiplicity one classes of minimal submanifolds of an arbitrary complete Riemannian manifold; this extension is briefly discussed in $\S 7$.

The proof of the main technical lemma (Lemma 1) is based on a variant of the "blowup method", a technique going back to De Giorgi in his work on area minimizing hypersurfaces, and first used in a context where "integrability hypotheses" (like $\ddagger \ddagger$ of $\S 1$ ) play a role by Allard and Almgren [4] in their study of minimal submanifolds with isolated singularities. Crucial among the new ingredients needed to make the blowup method work in the present context of nonisolated singularities are the $L^{2}$-estimates given in $\S 3$. It is also necessary to take care of the analysis of the solutions of the Jacobi field operator over cylindrical domains. This is somewhat more subtle than the corresponding analysis for the case of isolated singularities, and is discussed in $\S 4$.

The applicability of Theorem 1 to the analysis of the singular set of a wider class of minimal submanifolds (e.g., to codimension 1 absolutely minimizing currents) is limited by the "integrability hypotheses" $\ddagger \ddagger$ of $\S 1$ for the cross section of the tangent cylinder. Thus an important question which remains to be settled is whether or not some version of Lemma 1 is valid without this hypothesis.

\section{Notation and statement of main theorems}

$k, l, m, n$ will denote fixed positive integers with $n=l+m \geq 2$. $n$ will be the dimension of the submanifolds which we study, $k$ the codimension, and $l$ will be the "cross-sectional" dimension of the cylindrical tangent cones, as described below.

$B_{\rho}^{N}(X)$ denotes the open ball with center $X$ and radius $\rho$ in $\mathbf{R}^{N}$; $B_{\rho}(X), B_{\rho}$ will often be used as abbreviations for $B_{\rho}^{n+k}(X), B_{\rho}^{n+k}(0)$ respectively. 
$\eta_{Y, \rho}$ will the denote the map $X \mapsto \rho^{-1}(X-Y)$. Thus $\eta_{Y, \rho}$ translates $Y$ to the origin and homotheties by a factor $\rho^{-1}$.

$\mathscr{H}^{j}$ will denote $j$-dimensional Hausdorff measure.

$\mathscr{M}$ will denote a set of smooth $n$-dimensional minimal submanifolds; each $M \in \mathscr{M}$ is assumed properly embedded in $\mathbf{R}^{n+k}$ in the sense that for each $X \in M$ there is $\sigma>0$ such that $M \cap \bar{B}_{\sigma}(X)$ is a compact connected embedded smooth submanifold with boundary contained in $\partial B_{\sigma}(X)$. We also assume that for each $M \in \mathscr{M}$ there is a corresponding open set $U_{M} \supset M$, such that $\mathscr{H}^{n}(M \cap K)<\infty$ for each $M \in \mathscr{M}$ and each compact $K \subset U_{M}$, and such that $M$ is stationary in $U_{M}$ in the sense that

$$
\int_{M} \operatorname{div}_{M} \Phi d \mu=0
$$

whenever $\Phi=\left(\Phi^{1}, \cdots, \Phi^{n+k}\right): U_{M} \rightarrow \mathbf{R}^{n+k}$ is a $C^{\infty}$ vector field with compact support in $U_{M}$. Here $d \mu$ denotes integration with respect to ordinary $n$-dimensional volume measure (i.e., $n$-dimensional Hausdorff measure) on $M$, and $\operatorname{div}_{M} \Phi$ is the "tangential divergence" of $\Phi$ relative to $M$. Thus

$$
\operatorname{div}_{M} \Phi=\sum_{j=1}^{n+k}\left(e_{j} \cdot \nabla^{M}\right) \Phi^{j}
$$

where $e_{1}, \cdots, e_{n+k}$ is the standard basis for $\mathbf{R}^{n+k}$, and $\nabla^{M}$ denotes tangential gradient operator on $M$, so that if $f \in C^{1}(U)$ then $\nabla^{M} f(X)=$ $P_{X}\left(\operatorname{grad}_{\mathbf{R}^{n+k}} f(X)\right)$, with $P_{X}$ the orthogonal projection of $\mathbf{R}^{n+k}$ onto the tangent space $T_{X} M$ for any $X \in M$.

We assume that the $M \in \mathscr{M}$ have no removable singularities: thus if $X \in \bar{M} \cap U_{M}$, and there is $\sigma>0$ such that $\bar{M} \cap \bar{B}_{\sigma}(X)$ is a smooth compact connected embedded minimal submanifold with boundary contained in $\partial B_{\sigma}(X)$, then $X \in M$. Subject to this agreement, the (interior) singular set of $M$ (relative to $U_{M}$ ) is then defined by

$$
\operatorname{sing} M=U_{M} \cap \bar{M} \backslash M,
$$

and the regular set $\operatorname{reg} M$ is just $M$ itself.

We assume here also that the class $\mathscr{M}$ is closed under appropriate homotheties, rigid motions, and weak limits-we shall call such a class a "multiplicity one class"; more precisely, we assume the following:

1.3(a). $\quad M \in \mathscr{M} \Rightarrow q \circ \eta_{X, \rho} M \in \mathscr{M}$ and $q \circ \eta_{X, \rho} U_{M}=U_{q \circ \eta_{X, \rho} M}$ for each $X \in U_{M}$, each $\rho>0$, and each orthogonal transformation $q$ of $\mathbf{R}^{n+k}$. 
1.3(b). If $\left\{M_{j}\right\} \subset \mathscr{M}$, if $U \subset \mathbf{R}^{n+k}$ with $U \subset U_{M_{j}}$ for all sufficiently large $j$, and $\sup _{j \geq 1} \mathscr{H}^{n}\left(M_{j} \cap K\right)<\infty$ for each compact $K \subset U$, then there is a subsequence $M_{j^{\prime}}$ and an $M \in \mathscr{M}$ such that $U_{M_{j}} \supset U$ and $M_{j^{\prime}} \rightarrow M$ in $U$ in the sense that $\int_{M_{j^{\prime}}} f(X) d \mathscr{\mathscr { C }}^{n}(X) \rightarrow \int_{M} f(X) d \mathscr{H}^{n}(X)$ for any fixed continuous $f: \mathbf{R}^{n+k} \rightarrow \mathbf{R}$ with compact support in $U$.

Notice that $1.3(\mathrm{~b})$ is a strong restriction in that it precludes, in particular, the possibility of getting varifolds with multiplicity greater than one on a set of positive measure as the varifold limit of a sequence $M_{j} \subset \mathscr{M}$ with each $U_{M_{j}} \supset U$ for some fixed open $U$; for this reason we refer to such a class as a multiplicity 1 class.

1.4. Examples. In view of later applications, we should mention here a couple of important classes $\mathscr{M}$ which satisfy the conditions imposed above. One such class consists of the interior regular sets of the $\bmod 2$ minimizing currents described as follows: If $T$ is an $n$-dimensional locally rectifiable multiplicity 1 current in $\mathbf{R}^{n+k}$, if $\operatorname{spt}_{2} \partial T$ denotes the $\bmod 2$ support of $\partial T$, if $T$ is $\bmod 2$ minimizing in $\mathbf{R}^{n+k}$ (in the sense that for each bounded open $U \subset \mathbf{R}^{n+k}$ the mass of $T L U$ is $\leq$ the mass of $S\llcorner U$ for any multiplicity 1 current $S$ such that support of $T-S$ is a compact subset of $U$ and such that $T-S$ has zero mod 2 boundary in $U$ ), and if $\operatorname{reg}_{2} T$ is the mod 2 regular set of $T$ defined in the usual way as the set of all $X \in \operatorname{spt} T \backslash$ spt $_{2} \partial T$ such that $T$ is $\bmod 2$ equivalent in a neighborhood of $X$ to multiplicity one integration over a smooth properly embedded $n$-dimensional submanifold containing $X$, then the collection $\mathscr{T}_{2}$ of all such sets $M=\operatorname{reg}_{2} T$ is a class $\mathscr{M}$ satisfying all the conditions imposed above, provided we take $U_{M}=\mathbf{R}^{n+k} \backslash \mathrm{spt}_{2} \partial T$. Indeed by the Allard theorem spt $T \backslash\left(\operatorname{reg} T \cup \operatorname{spt}_{2} \partial T\right)$ has $\mathscr{l}^{n}$-measure zero, and it follows that $M=\operatorname{reg}_{2} T$ satisfies (1.1), and, using the notation introduced above in our discussion of the general class $\mathscr{M}$, we have $\operatorname{sing} M=\operatorname{spt} T \backslash\left(\operatorname{reg} T \cup \operatorname{spt}_{2} \partial T\right)$, which coincides with the usual definition of the (interior) singular set of such $\bmod 2$ minimizing currents $T$. The property 1.3(b) (plus an existence theory) is true by the compactness theorem for flat chains $\bmod p$ (see, e.g. [11] or [9]).

Another such class is the collection $\mathscr{T}_{3}=\left\{\operatorname{reg}_{3} T\right\}$ of the interior regular sets of $n$-dimensional multiplicity 1 currents $T$ which are $\bmod 3$ minimizing in $\mathbf{R}^{n+k}$ (defined analogously to the $\bmod 2$ case); if $M=$ $\operatorname{reg}_{3} T$ then $M$ satisfies (1.1) with $U_{M}=\mathbf{R}^{n+k} \backslash \operatorname{spt}_{3} \partial T$, and $\operatorname{sing} M=$ spt $T \backslash\left(\operatorname{reg} T \cup \mathrm{spt}_{3} \partial T\right)$. Again the property 1.3(b) (plus an existence 
theory) is true by the compactness theorem for flat chains $\bmod p$.

Notice that these classes $\mathscr{T}_{2}, \mathscr{T}_{3}$ have $\operatorname{dim} \operatorname{sing} M \leq(n-2),(n-1)$ respectively by [10], [6].

A third class which has the form of $\mathscr{M}$ above is the collection $\mathscr{T}$ of all submanifolds $M$ of the form $M=\operatorname{reg} T$, where $T$ is an $n$-dimensional orientated boundary of least area in some open $U=U_{T} \subset \mathbf{R}^{n+k}$, in the usual sense that $T=\partial \llbracket V \rrbracket$ in $U$ (in the sense of currents) for some measurable $V \subset U$ and $T L U$ has mass $\leq$ than the mass of $S\llcorner U$, for any multiplicity 1 locally rectifiable current $S$ in $\mathbf{R}^{n+k}$ with support $S-T$ equal to a compact subset of $U$ and with $\partial(S-T)=0$ in $U$. In this case, with $M=\operatorname{reg} T$, we take $U_{M}=U, \operatorname{sing} M=U \cap \operatorname{spt} T \backslash(\operatorname{reg} T \cup \operatorname{spt} \partial T)$, and the singular set satisfies $\operatorname{dim} \operatorname{sing} M \backslash \operatorname{spt} \partial T \leq n-7$ (see, e.g., [10] or [19] or [13]). The property 1.3(b) in this case is discussed in, e.g., [9], [13] or [19].

For the first class mentioned above (i.e., $\mathscr{T}_{2}$ ), Theorem 2 implies countable $(n-2)$-rectifiability for $\operatorname{sing} M$ and a local finiteness result for the $(n-2)$-dimensional measure of measure $\operatorname{sing} M$ in Corollary 1 of $\S 1$. (See the precise statement in Corollary 3.) Corollary 2 gives information about the singular set of the class $\mathscr{T}_{3}$, and analogous classes of stationary surfaces, in the case $n=2$. Application to the third class $\mathscr{T}$ (the absolutely minimizing multiplicity one currents) is hampered by the integrability hypothesis $\ddagger$.

We briefly need to recall here some basic properties of minimal submanifolds in the context of our general class $\mathscr{M}$. First recall that, by the monotonicity formula, for each $M \in \mathscr{M}$,

$$
\rho^{-n} \mathscr{H}^{n}\left(M \cap B_{\rho}(X)\right)
$$

is increasing as a function of $\rho$ so long as $\bar{B}_{\rho}(X) \subset U_{M}$, and hence in particular the density

$$
\Theta_{M}(X)=\lim _{\rho \downarrow 0} \frac{\mathscr{H}^{n}\left(M \cap B_{\rho}(X)\right)}{\omega_{n} \rho^{n}},
$$

where $\omega_{n}$ is the volume of the unit ball in $\mathbf{R}^{n}$, exists and is upper semicontinuous on $U_{M}$. Notice that then $\bar{M} \cap U_{M}$ is just the set of points $X \in U_{M}$ with $\Theta_{M}(X) \geq 1$, and, by (1.5),

$$
\mathscr{H}^{n}\left(M \cap B_{\rho}(X)\right) \geq \omega_{n} \rho^{n}, \quad X \in \bar{M}, B_{\rho}(X) \subset U_{M} .
$$

This shows that $\mathscr{H}^{n}(\operatorname{sing} M)=0$ for each $M \in \mathscr{M}$, because by measure theory (e.g., [19, Theorem 3.5] or [9]) we know that $\lim _{\rho \downarrow 0} \rho^{-n} \mathscr{H}^{n}\left(M \cap B_{\rho}(X)\right)=0$ for $\mathscr{H}^{n}$-a.e. $X \in U_{M} \backslash M$. If we use 
the Allard regularity theorem [1], we get the additional fact that $M$ coincides precisely with the set of $X \in U_{M}$ with $\Theta_{M}(X)=1$; in fact the Allard theorem guarantees that there is $\alpha_{0}=\alpha_{0}(n, k)>0$ such that

$$
\begin{aligned}
& M=\left\{X \in U_{M}: 0<\Theta_{M}(X)<1+\alpha_{0}\right\}, \\
& \operatorname{sing} M=\left\{X \in U_{M}: \Theta_{M}(X) \geq 1+\alpha_{0}\right\} .
\end{aligned}
$$

Also, by a simple compactness argument based on (1.3), (1.7), and (1.5), it is straightforward to check the following: For each $\Lambda>0$, there is $\beta=\beta(\mathscr{M}, \Lambda)>0$ such that if $M \in \mathscr{M}, \rho>0, B_{\rho} \subset U_{M}$, $\mathscr{H}^{n}\left(M \cap B_{\rho}\right) \leq \Lambda, M \cap B_{3 \rho / 4} \neq \varnothing$, and $\rho^{-n-2} \int_{M \cap B_{\rho}} \operatorname{dist}^{2}(X, P)<\beta$ for some $n$-dimensional subspace $P \subset \mathbf{R}^{n+k}$, then there is a $C^{2}$-map $u: P \cap B_{3 \rho / 4} \rightarrow P^{\perp}$ such that

$$
M \cap B_{\rho / 2} \subset \text { graph } u \subset M,
$$

$$
\rho^{-2} \sup |u|^{2}+\sup |\nabla u|^{2} \leq C \rho^{-n-2} \int_{B_{\rho} \cap M} \operatorname{dist}^{2}(X, P) d \mu .
$$

Another important general fact (see, e.g., [1], [19]) concerns tangent cones: if $M \in \mathscr{M}$ and $X \in \bar{M} \cap U_{M}$, and if $\rho_{j} \downarrow 0$, then there is a subsequence $\rho_{j^{\prime}}$ such that $\eta_{X, \rho_{j^{\prime}}} M$ tends in the sense of 1.3(b) to a cone $\mathbf{C} \in \mathscr{M}$ with vertex at 0 . Thus $U_{\mathbf{C}}=\mathbf{R}^{n+k}$ and $\eta_{0, \lambda} \mathbf{C}=\mathbf{C}$ for every $\lambda>0$. The set of all such $\mathbf{C}$ is denoted subsequently by $\operatorname{Tan}_{X} M$; notice that $\operatorname{Tan}_{X} M$ is a singleton set containing just the classical tangent plane of $M$ at $X$ if $X \in M$, but that it is far from obvious (and an open question) whether or not $\operatorname{Tan}_{X} M$ can contain more than one cone $\mathbf{C}$ if $X \in \operatorname{sing} M$. We note also that $\Theta_{M}(X)=\Theta_{\mathbf{C}}(0)$ for each $\mathbf{C} \in \operatorname{Tan}_{X} M$, again by (1.5) and $1.3(\mathrm{~b})$.

An important property of $\boldsymbol{\Theta}_{\mathbf{C}}$ for cones $\mathbf{C} \in \mathscr{M}$ (i.e., elements $\mathbf{C} \in \mathscr{M}$ with $\left.\eta_{0, \lambda} \mathbf{C}=\mathbf{C} \forall \lambda>0\right)$ is that

(1.9) $S(\mathbf{C}) \equiv\left\{Y \in \mathbf{R}^{n+k}: \boldsymbol{\Theta}_{\mathbf{C}}(Y)=\boldsymbol{\Theta}_{\mathbf{C}}(0)\right\}$ is a linear subspace of $\mathbf{R}^{n+k}$.

(Notice of course that by upper semicontinuity of $\Theta_{\mathbf{C}}$, it is automatic that $\Theta_{\mathbf{C}}(Y) \leq \Theta_{\mathbf{C}}(0)$ for every $Y \in \mathbf{R}^{n+k}$.) (1.9) follows from the fact that, by a standard argument based on the monotonicity formula (see [1] or [19]), if $Y \in S(\mathbf{C})$ then $\mathbf{C}$ is also a cone with vertex at $Y$, so in particular $\Theta_{\mathbf{C}}(Y+\lambda X)=\Theta_{\mathbf{C}}(Y+X)$ for each $X \in \mathbf{R}^{n+k}$ and each $\lambda>0$. Thus for any $\lambda>0$, any $X \in \mathbf{R}^{n+k}$, and any $Y \in S(\mathbf{C})$, we have

$$
\begin{aligned}
\Theta_{\mathbf{C}}(X) & =\Theta_{\mathbf{C}}(\lambda X)=\Theta_{\mathbf{C}}(Y+\lambda X-Y)=\Theta_{\mathbf{C}}\left(Y+\lambda^{-2}(\lambda X-Y)\right) \\
& =\Theta_{\mathbf{C}}\left(\lambda Y+\lambda^{-1}(\lambda X-Y)\right)=\Theta_{\mathbf{C}}\left(X+\left(\lambda-\lambda^{-1}\right) Y\right),
\end{aligned}
$$


so that $\Theta_{\mathbf{C}}(X+t Y)=\Theta_{\mathbf{C}}(X)$ for every $t \in \mathbf{R}, X \in \mathbf{R}^{n+k}$ and every $Y \in S(C)$, and the fact that $S(\mathbf{C})$ is a linear subspace follows directly from this. Of course (for example by (1.7) with $M=\mathrm{C}$ ), it is then automatic that $\mathbf{C}$ is actually invariant under translations by elements of $S(\mathrm{C})$, so that

$$
\eta_{Y, \lambda} \mathbf{C}=\mathbf{C} \quad \forall Y \in S(\mathbf{C}), \quad \lambda>0 .
$$

Since $\mathscr{M}$ is a multiplicity 1 class (so that in particular $1.3(\mathrm{~b})$ holds and each $\mathbf{C} \in \operatorname{Tan}_{X} M$ must be multiplicity 1 -in fact it lies in $\mathscr{M}$ ), using (1.8) we easily check that the following alternative characterization of $M$ holds:

$$
M=\left\{X \in U \cap \bar{M}: \exists C \in \operatorname{Tan}_{X} M \text { with } \operatorname{dim} S(\mathbf{C})=n\right\} .
$$

Of course $\operatorname{dim} S(\mathbf{C})=n$ means precisely that $\mathbf{C}$ is an $n$-dimensional subspace of $\mathbf{R}^{n+k}$-in fact just the classical tangent space of $M$ at $X$ once it is established that $X \in M$.

We shall also need the following useful "stratification of $\operatorname{sing} M$ by tangent cone type", which is a direct modification of $[7,2.28]$ to the present context. Namely, if

$\mathscr{S}_{j}(M)=\left\{X \in \operatorname{sing} M: \operatorname{dim} S(\mathbf{C}) \leq j \forall \mathbf{C} \in \operatorname{Tan}_{X} M\right\}, \quad j=0, \cdots, n-1$, then

$$
\begin{gathered}
\text { Hausdorff dimension of } \mathscr{S}_{j}(M) \leq j, j=0,1, \ldots, n-1, \\
\mathscr{S}_{0}(M) \cap\left\{X: \Theta_{M}(X)=\alpha\right\} \text { is discrete for each } \alpha .
\end{gathered}
$$

To prove the first part of (1.10) for $j=0,1, \cdots, n-1$, we first note that, by (1.5), (1.9), and the definition of $\mathscr{S}_{j}(M)$, for each $X \in \mathscr{S}_{j}(M)$ and each $\delta>0$ there is an integer $\mu=\mu(X, \delta, M) \geq\left(\operatorname{dist}\left(X, \partial U_{M}\right)\right)^{-1}$ such that, whenever $\sigma \in(0,1 / \mu), \eta_{X, \sigma}\left\{Y \in B_{\sigma}(X): \Theta_{M}(Y) \geq \Theta_{M}(X)-\right.$ $1 / \mu\} \quad\left(\equiv\left\{Y \in B_{1}: \Theta_{\eta_{X, \sigma} M}(Y) \geq \Theta_{\eta_{X, \sigma} M}(0)-1 / \mu\right\}\right)$ is contained in the $\delta$-neighborhood of some $j$-dimensional subspace $L_{X, \sigma}$ (depending on $X, \sigma)$. With $\delta>0$ arbitrary (and fixed), let

$$
\mathscr{S}_{j, i}=\left\{X \in \mathscr{S}_{j}(M): \mu(X, \delta, M)=i\right\},
$$

and for $q>i$ let

$$
\mathscr{S}_{j, i, q}=\left\{X \in \mathscr{S}_{j, i}: \Theta_{M}(X) \in[(q-1) / i, q / i)\right\} .
$$

Notice that then for any given $X \in \mathscr{S}_{j, i, q}$ we have

$$
\mathscr{S}_{j, i, q} \subset\left\{Y \in \mathscr{S}_{j, i}: \Theta_{M}(Y) \geq \Theta_{M}(X)-1 / i\right\},
$$


and hence, for each $\sigma>0$,

$$
\eta_{X, \sigma}\left(\mathscr{S}_{j, i, q} \cap B_{\sigma}(X)\right) \subset \eta_{X, \sigma}\left\{Y \in B_{\sigma}(X): \Theta_{M}(Y) \geq \Theta_{M}(X)-1 / i\right\},
$$

which is contained in the $\delta$-neighborhood of the $j$-dimensional subspace $L_{X, \sigma}$ (as above) for each $\sigma \in(0,1 / i)$.

On the other hand, a simple iterative argument using the definition of Hausdorff measure shows directly that if $A$ is any subset of $\mathbf{R}^{n+k}$ and if for each $X \in A$ there is some $\sigma_{0}=\sigma_{0}(X)>0$ such that $\eta_{X, \sigma}\left(A \cap B_{\sigma}(X)\right)$ is contained in the $\delta$-neighborhood of some $j$-dimensional subspace of $\mathbf{R}^{n+k}$, depending on $X, \sigma$ whenever $X \in A$ and $\sigma<\sigma_{0}$, then $\mathscr{H}^{j+\theta(\delta)}(A)=0$ for some $\theta(\delta)$ depending only on $\delta, n, k$, with $\theta(\delta) \downarrow 0$ as $\delta \downarrow 0$. Since each $\mathscr{S}_{j, i, q}$ has such a property (as proved above), this shows $\mathscr{H}^{j+\theta(\delta)}\left(\mathscr{S}_{j}\right) \equiv \mathscr{H}^{j+\theta(\delta)}\left(\bigcup_{i \geq 1} \bigcup_{q>i} \mathscr{S}_{j, i, q}\right)=0$ for every $\delta>0$, thus completing the proof of (1.10) for $j=1, \cdots, n-1$.

The fact that $\mathscr{S}_{0} \cap\left\{X: \Theta_{M}(X)=\alpha\right\}$ is discrete is easier: if not, we get $X_{0} \in \mathscr{S}_{0}$ such that $X_{j} \rightarrow X_{0}$ for some sequence $\left\{X_{j}\right\} \subset \mathscr{S}_{0} \backslash\left\{X_{0}\right\}$ with $\Theta_{M}\left(X_{j}\right)=\alpha \forall j$. Using 1.3(b) to take a convergent subsequence of $\eta_{X_{0},\left|X_{0}-X_{j}\right|} M$, and using (1.7) we then get $\mathbf{C} \in \operatorname{Tan}_{X_{0}} M$ such that sing $\mathbf{C} \cap$ $S^{n+k-1} \neq \varnothing$, contradicting the fact that $X_{0} \in \mathscr{S}_{0}$.

From now on, $\mathbf{C}^{(0)} \in \mathscr{M}$ will denote a cylinder of the form $\mathbf{C}^{(0)}=$ $\mathbf{C}_{0}^{(0)} \times \mathbf{R}^{m}$, where $\mathbf{C}_{0}^{(0)}$ is stationary in $\mathbf{R}^{l+k}$ and sing $\mathbf{C}_{0}^{(0)}=\{0\}$. We also let $\Sigma_{0}=\mathbf{C}_{0}^{(0)} \cap S^{l+k-1}$, so that $\Sigma_{0}$ is a smooth compact $(l-1)$-dimensional submanifold of $S^{l+k-1}$ if $l \geq 2$ and a finite set of points if $l=1$.

The following classes will be referred to frequently in what follows:

1.11. Definition. $\mathscr{N}_{\varepsilon}\left(\mathbf{C}^{(0)}\right)$ is the set of all $M \in \mathscr{M}$ (where $\mathscr{M}$ satisfies all conditions discussed above, including (1.2), (1.3)) such that $U_{M} \supset$ $\bar{B}_{1}, \int_{M \cap B_{1}} \operatorname{dist}^{2}\left(X, C^{(0)}\right)+\int_{C^{(0)} \cap B_{1}} \operatorname{dist}^{2}(X, M)$ and $\mathscr{H}^{n}\left(M \cap B_{1}\right) \leq 1+$ $\mathscr{H}^{n}\left(\mathbf{C}^{(0)} \cap B_{1}\right)$.

Remarks. (1) Using (1.5), the compactness 1.3(b), and (1.8) (applied to translations and homotheties of $M$ ) it is easy to check that there is $\sigma:(0,1) \rightarrow(0, \infty)$, depending only on $\mathscr{M}$ and $\mathbf{C}^{(0)}$, with $\sigma(\varepsilon) \downarrow 0$ as $\varepsilon \downarrow 0$ and such that

$$
M \in \mathscr{N}_{\varepsilon}\left(\mathbf{C}^{(0)}\right) \Rightarrow\left|\mathscr{H}^{n}\left(M \cap B_{\rho}\right)-\mathscr{H}^{n}\left(\mathbf{C}^{(0)} \cap B_{\rho}\right)\right| \leq \sigma(\varepsilon) \rho^{n}
$$

for $\sigma(\varepsilon)<\rho<1-\sigma(\varepsilon)$. 
(2) For given $\varepsilon>0$ and any given $M \in \mathscr{M}$, if $\mathbf{C}^{(0)} \in \operatorname{Tan}_{X} M$ then automatically $\eta_{X, \rho_{j}} M \in \mathscr{N}_{\varepsilon}\left(\mathbf{C}^{(0)}\right)$ for some sequence of $\rho_{j} \downarrow 0$; this follows directly from the definition of $\operatorname{Tan}_{X} M, 1.5$, and 1.3(b).

1.12. Definition. (i) $\mathscr{C}_{\varepsilon}\left(\mathbf{C}^{(0)}\right)$ is the set of all cylinders of the form $\mathbf{C}=\mathbf{C}_{0} \times \mathbf{R}^{m} \subset \mathbf{R}^{l+k}$, where $\mathbf{C}_{0} \subset \mathscr{H}^{l}\left(\mathbf{C}_{0}\right) \leq 1+\mathscr{H}^{l}\left(\mathbf{C}_{0}^{(0)}\right)$ is a cone with sing $\mathbf{C}_{0}=\{0\}$, with $\Sigma \equiv \mathbf{C}_{0} \cap S^{l+k-1}$ smooth and compact for $l \geq 2$ and a finite set of points for $l=1$, with $\mathbf{C}_{0}$ stationary in $\mathbf{R}^{l+k} \backslash\{0\}$, $\mathscr{H}^{l}\left(\mathbf{C}_{0}\right) \leq 1+\mathscr{H}^{l}\left(\mathbf{C}_{0}^{(0)}\right)$ and

$$
\int_{\mathbf{C} \cap B_{1}} \operatorname{dist}^{2}\left(X, \mathbf{C}^{(0)}\right)+\int_{\mathbf{C}^{(0)} \cap B_{1}} \operatorname{dist}^{2}(X, \mathbf{C}) \leq \varepsilon^{2} .
$$

(ii) $\tilde{\mathscr{C}}_{\varepsilon}\left(\mathbf{C}^{(0)}\right)$ denotes the subset of the set of orthogonal transformations of elements of $\mathscr{C}_{\varepsilon}\left(\mathbf{C}^{(0)}\right)$ given by $\tilde{\mathscr{C}}_{\varepsilon}\left(\mathbf{C}^{(0)}\right)=\left\{\exp (A) \mathbf{C}: \mathbf{C} \in \mathscr{E}_{\varepsilon}\left(\mathbf{C}^{(0)}\right)\right.$, $A \in \mathscr{S}$ and $|A|<\varepsilon\}$, where $\mathscr{S}$ denotes the subspace of skew-symmetric transformations of $\mathbf{R}^{n+k}$ spanned by the special skew-symmetric transformations $(x, y) \mapsto x^{i} e_{l+k+j}-y^{j} e_{i},(x, y) \in \mathbf{R}^{l+k} \times \mathbf{R}^{m}, i=1, \cdots, l+k$, $j=1, \cdots, m$.

Note. It is not assumed that $\mathscr{C}_{\varepsilon}\left(\mathbf{C}^{(0)}\right) \subset \mathscr{M}$.

For any open $\Omega \subset \mathbf{C}$ where $\mathbf{C}=\mathbf{C}_{0} \times \mathbf{R}^{m} \in \mathscr{C}_{\varepsilon}\left(\mathbf{C}^{(0)}\right.$ for some $\left.\varepsilon>0\right)$, we let $C^{2}\left(\Omega ; \mathbf{C}^{\perp}\right)$ denote the maps $u \in C^{2}\left(\Omega ; \mathbf{R}^{n+k}\right)$ such that $u(x, y) \in$ $\left(T_{(x, y)} \mathbf{C}\right)^{\perp}$ for each $(x, y) \in \Omega$, and $C^{2}\left(\Omega ; \mathbf{C}_{0}^{\perp}\right)$ is defined analogously for any open $\Omega \subset \mathbf{C}_{0}$. Thus $C^{2}\left(\Omega ; \mathbf{C}^{\perp}\right)$ is just the set of $C^{2}$ sections of the normal bundle over $\Omega \subset \mathbf{C}$, and $C^{2}\left(\Omega ; \mathbf{C}_{0}^{\perp}\right)$ is the set of $C^{2}$ sections of the normal bundle over $\Omega \subset \mathbf{C}_{0} . \Delta_{\mathbf{C}}$ and $\Delta_{\mathbf{C}_{0}}$ will be the usual (normal) Laplacian operators on such normal sections. For $u \in C^{2}\left(\Omega ; \mathbf{C}^{\perp}\right)$ we define

$$
\text { graph } u=\{(x, y)+u(x, y):(x, y) \in \mathbf{C}\} .
$$

We let $\mathscr{Q}_{\mathbf{C}}, \mathscr{Q}_{\mathbf{C}_{0}}$ and note the minimal surface operators on $C^{2}\left(\mathbf{C} ; \mathbf{C}^{\perp}\right)$ and $C^{2}\left(\mathbf{C}_{0} ; \mathbf{C}_{0}^{\perp}\right)$ respectively (i.e., the Euler-Lagrange operators for the area functional of graphs of normal sections over domains in $\mathbf{C}$ and $\mathbf{C}_{0}$ respectively), and let $\mathscr{L}_{\mathbf{C}}, \mathscr{L}_{\mathbf{C}_{0}}$ be the linearizations of $\mathscr{Q}_{\mathbf{C}}, \mathscr{Q}_{\mathbf{C}_{0}}$ at 0 . Thus using variables $(x, y)=(r \omega, y) \in \mathbf{R}^{n} \times \mathbf{R}^{m}, r=|x|, \omega=|x|^{-1} x$, and letting $\Delta_{y}$ be the Laplacian $\sum_{j=1}^{m}\left(\partial / \partial y^{j}\right)^{2}$ in the $y$-variables, we have

$$
\mathscr{L}_{\mathbf{C}} v=\Delta_{y} v+\mathscr{L}_{\mathbf{C}_{0}} v
$$


with

$$
\begin{aligned}
\mathscr{L}_{\mathbf{C}_{0}} v & =\Delta_{\mathbf{C}_{0}} v+\frac{q(\omega)}{r^{2}} v \\
& =\frac{1}{r^{l-1}} \frac{\partial}{\partial r}\left(r^{l-1} \frac{\partial v}{\partial r}\right)+\frac{1}{r^{2}}\left(\Delta_{\Sigma} v+q(\omega) v\right),
\end{aligned}
$$

where $q(\omega):\left(T_{\omega} \Sigma\right)^{\perp} \rightarrow\left(T_{\omega} \Sigma\right)^{\perp}$ is the zero-order operator given by $\langle w, q(\omega)(v)\rangle=\operatorname{trace} A_{\omega}(w)^{*} \circ A_{\omega}(v)$, for any $v, w \in\left(T_{\omega} \Sigma\right)^{\perp}$, with $A_{\omega}$ the second fundamental form of $\Sigma$ (thought of as an operator from $\left(T_{\omega} \Sigma\right)^{\perp} \rightarrow \operatorname{Hom}\left(T_{\omega} \Sigma, T_{\omega} \Sigma\right)$ in the usual way). The operator $\Delta_{\Sigma}+q(\omega)$ appearing here will henceforth be denoted $\mathscr{L}_{\Sigma}$; notice that $\mathscr{L}_{\Sigma}$ is a selfadjoint elliptic operator on the normal sections $C^{2}\left(\Sigma ; \mathbf{C}_{0}^{\perp}\right)$ of the normal bundle of the smooth compact submanifold $\Sigma$ of $S^{n+k-1}$, so the eigenvalues of $-\mathscr{L}_{\Sigma}$ form a sequence

$$
\lambda_{1} \leq \lambda_{2} \leq \lambda_{3} \leq \cdots \leq \lambda_{k} \leq \cdots, \quad \lambda_{k} \uparrow \infty,
$$

and there is a corresponding complete orthonormal sequence of eigenfunctions $\varphi_{1}, \varphi_{2}, \varphi_{3}, \cdots, \varphi_{k}, \cdots$ (orthonormal with respect to the $L^{2}(\Sigma)$ norm).

There is an important method of generating solutions of the equation $\mathscr{L}_{\mathbf{C}_{0}} v=0$, analogous to the method in Riemannian geometry of generating Jacobi fields by taking the initial velocity vector of a one-parameter family of geodesics, as follows:

Suppose $\left\{M_{t}\right\}_{\{t \in(-1,1)\}}$ is a one-parameter family of $l$-dimensional minimal submanifolds in $\mathbf{R}^{l+k}$ with $M_{0}=\mathbf{C}_{0}$, and suppose that, for small enough $t, M_{t}$ can be expressed as the graph, over a domain $\Omega_{t} \subset \mathbf{C}_{0}$, of a $C^{2}\left(\Omega_{t} ; \mathbf{C}_{0}^{\perp}\right)$ function $u_{t}$; thus $u_{t}$ is a smooth section of the normal bundle of $\mathbf{C}_{0}$ over $\Omega_{t}$ and $M_{t}=$ graph $u_{t} \equiv\left\{x+u_{t}(x): x \in \Omega_{t}\right\}$. Suppose also that $u_{t}(x)$ depends in a $C^{1}$ fashion jointly in $(x, t)$, and that the $\Omega_{t}$ engulf all of $\mathbf{C}_{0}$ as $|t| \downarrow 0$ in the sense that $\bigcup_{s>0} \bigcap_{0<|t|<s} \Omega_{t}=\mathbf{C}_{0}$. Let $v$ be the initial velocity given by $v=\partial u_{t} /\left.\partial t\right|_{t=0}$. Then $v$ satisfies the equation $\mathscr{L}_{\mathrm{C}_{0}} v=0$ on all of $\mathrm{C}_{0}$. An important special case of this general principle is as follows:

1.13. Example. Assume that the family $\left\{M_{t}\right\}$, satisfying the general conditions imposed in the above discussion, is a family of minimal cones $\left\{\mathbf{C}_{t}\right\}$ all with sing $\mathbf{C}_{t}=\{0\}$. In this case the initial velocity $v$ is a function which is homogeneous of degree 1 in the variable $r=|x|$; that is, $v=$ $r \varphi(\omega)$, where $r=|x|$ and $\omega=|x|^{-1} x$. 
Remark. Such families $\mathbf{C}_{t}$ can be obtained for example by rotations of $\mathbf{C}_{0}$; thus $\mathbf{C}_{t}=(\exp t A) \mathbf{C}_{0}$, where $A$ is any fixed $n \times n$ skew symmetric matrix, gives such a family. In this case we get $v(r, \omega)=r(A \omega)^{\perp}, \omega \in$ $\mathbf{C}_{0}$, where ()$^{\perp}$ means orthogonal projection onto $\left(T_{\omega} \mathbf{C}_{0}\right)^{\perp}$.

In the main technical lemma, which we now state, we need an additional condition on $\mathbf{C}_{0}^{(0)}$ (like the "integrability condition" needed in [4]) as follows:

Every homogeneous degree 1 solution of $\mathscr{L}_{\mathbf{C}_{0}^{(0)}} v=0$ is generated, in the manner described in Example 1.13, by a 1-parameter family $\left\{\mathbf{C}_{t}\right\}_{|t|<1}$ of minimal cones, all stationary in $\mathbf{R}^{l+k} \backslash\{0\}$, with sing $\mathbf{C}_{t}=\{0\}$ and $\mathbf{C}_{0}=\mathbf{C}_{0}^{(0)}$.

We can now state the main technical lemma of the present paper; all the main results about the structure of the singular set proved here are based on this lemma. The proof of the lemma will be given in $\S 5$.

Lemma 1. Suppose that $\mathbf{C}_{0}^{(0)}$ satisfies $\ddagger \ddagger$. For any $\theta \in\left(0, \frac{1}{4}\right)$, there are $\delta_{0}=\delta_{0}\left(\mathbf{C}^{(0)}, \theta\right), \varepsilon_{0}=\varepsilon_{0}\left(\mathbf{C}^{(0)}, \mathscr{M}, \theta\right) \in\left(0, \frac{1}{4}\right)$ such that if $M \in \mathscr{N}_{\varepsilon_{0}}\left(\mathbf{C}^{(0)}\right)$ and $\mathbf{C} \in \tilde{\mathscr{C}}_{\varepsilon_{0}}\left(\mathbf{C}^{(0)}\right)$, then either

$$
B_{\delta_{0}}(0, y) \cap\left\{X \in B_{1}: \Theta_{M}(X) \geq \Theta_{\mathbf{C}^{(0)}}(0)\right\}=\varnothing \quad \text { for some } y \in B_{1 / 2}^{m}(0),
$$

or there is a $\widetilde{\mathbf{C}} \in \widetilde{\mathscr{C}}_{\gamma \varepsilon_{0}}\left(\mathbf{C}^{(0)}\right)$ such that

$$
\theta^{-n-2} \int_{M \cap B_{\theta}} \operatorname{dist}^{2}(X, \widetilde{\mathbf{C}}) \leq C \theta^{\alpha} \int_{M \cap B_{1}} \operatorname{dist}^{2}(X, \mathbf{C}),
$$

where $C=C\left(\mathbf{C}^{(0)}, \mathscr{M}\right)>0, \gamma=\gamma\left(C^{(0)}, \mathscr{M}, \theta\right) \geq 1$, and $\alpha=\alpha\left(\mathbf{C}^{(0)}, \mathscr{M}\right)$ $\in(0,1)$.

1.14. Remark. For later reference we note that the alternative (i) not holding implies

$$
\{0\} \times\left\{y \in \mathbf{R}^{m}:|y| \leq \frac{1}{2}\right\} \subset \mathbf{B}_{2 \delta_{0}}\left(\left\{X: \boldsymbol{\Theta}_{M}(X) \geq \boldsymbol{\Theta}_{\mathbf{C}^{(0)}}(0)\right\}\right),
$$

where we use the notation that $\mathbf{B}_{\sigma}(S)=\bigcup_{X \in S} B_{\sigma}(X)$ for any $S \subset \mathbf{R}^{n+k}$ and $\sigma>0$.

Using Lemma 1 we will show in particular that the following "decomposition theorem" follows directly:

Theorem 1. Suppose $\mathbf{C}_{0}^{(0)}$ satisfies $\ddagger$. There are $\varepsilon=\varepsilon\left(\mathscr{M}, \mathbf{C}^{(0)}\right), \delta_{0}=$ $\delta_{0}\left(\mathscr{M}, \mathbf{C}^{(0)}\right), \alpha=\alpha\left(\mathscr{M}, \mathbf{C}^{(0)}\right) \in(0,1)$ such that if $M \in \mathscr{M}, U_{M} \supset B_{2}$, 
and

$$
\frac{\mathscr{P}^{n}\left(M \cap B_{2}\right)}{\omega_{n} 2^{n}}-\Theta_{C^{(0)}}(0)<\varepsilon^{2}, \quad \int_{M \cap B_{2}(0)} \operatorname{dist}^{2}\left(X, C^{(0)}\right)<\varepsilon^{2},
$$

then

$$
\left\{X \in B_{1}: \Theta_{M}(X) \geq \boldsymbol{\Theta}_{C^{(0)}}(0)\right\}=S \cup T,
$$

with $S \subset L, L$ a properly embedded $m$-dimensional $C^{1, \alpha}$ manifold with $\mathscr{H}^{m}(L) \leq \omega_{m}$, and with $T \subset \bigcup_{j} B_{\rho_{j}}\left(X_{j}\right)$ for some family of balls $B_{\rho_{j}}\left(X_{j}\right)$ such that $\sum_{j} \rho_{j}^{m} \leq 1-\delta_{0}$.

The proof of this will be given in $\S 5$.

Our first main theorem about the singular set, which we now state, follows more or less directly by iteration of the above decomposition theorem, as we show precisely in $\S 6$. In this theorem $m$ denotes the maximum among the positive integers $m$ such that there is a cylindrical tangent cone $\mathbf{C}_{0} \times \mathbf{R}^{m} \in \mathscr{M} . m$ is called the top dimension for the singular sets of $M \in \mathscr{M}$; using (1.10) it is easy to check that in fact

$$
m=\max \{\operatorname{dim} \operatorname{sing} M: M \in \mathscr{M}\},
$$

so the terminology is appropriate. Here and subsequently we also use the notation

$\operatorname{sing}_{\alpha} M=\left\{X: \boldsymbol{\Theta}_{M}(X)=\alpha\right.$ and $\exists$ a cylindrical cone $\left.q\left(\mathbf{C}_{0} \times \mathbf{R}^{m}\right) \in \operatorname{Tan}_{X} M\right\}$.

Theorem 2 (Main Structure Theorem). Suppose $m$ is as in (1.15). Suppose also that, for each cylindrical cone $\mathbf{C}^{(0)}=\mathbf{C}_{0}^{(0)} \times \mathbf{R}^{m} \in \mathscr{M}$, the cross section $\mathbf{C}_{0}^{(0)}$ satisfies the integrability condition $\ddagger$. Then for each $M \in \mathscr{M}$, $\operatorname{sing} M$ is countably $m$-rectifiable, and $\operatorname{sing}_{\alpha} M$ has locally finite $\mathscr{H}^{m}$ measure for each $\alpha$; further any compact $K \subset U_{M}$ intersects $\operatorname{sing}_{\alpha} M$ for at most finitely many $\alpha$.

Remarks. (1) $\operatorname{sing}_{\alpha} M$ has locally finite $\mathscr{H}^{m}$-measure in the usual sense that for each $X \in \operatorname{sing}_{\alpha} M$ there is $\rho=\rho(X)>0$ such that $\mathscr{H}^{m}\left(\operatorname{sing}_{\alpha} M \cap B_{\rho}(X)\right)<\infty$.

(2) Recall that by (1.10) $\operatorname{sing} M \backslash\left(\bigcup_{\alpha} \operatorname{sing}_{\alpha} M\right) \subset \bigcup_{j=0}^{m-1} \mathscr{S}_{j}(M)$, and hence has dimension $\leq m-1$ and is discrete for $m=1$.

(3) Note that it is automatically true that $\operatorname{sing} \mathbf{C}_{0}^{(0)} \subset\{0\}$ if $\mathbf{C}_{0}^{(0)}$ is a cone and $\mathbf{C}_{0}^{(0)} \times \mathbf{R}^{m} \subset \mathscr{M}$ (because otherwise $\mathbf{C}_{0}^{(0)}$ would contain a whole ray of singular points and then $\mathbf{C}_{0}^{(0)} \times \mathbf{R}^{m}$ would have singular set of dimensions at least $m+1$, contradicting (1.15)), so it makes sense to require that $\mathbf{C}_{0}^{(0)}$ satisfies $\ddagger$. 
Actually we prove in $\S 6$ a slightly stronger theorem.

Theorem $2^{\prime}$. Suppose $m$ is as in (1.15). Suppose also that $\alpha>1$ is given, and that all cylindrical cones $\mathbf{C}^{(0)}=\mathbf{C}_{0}^{(0)} \times \mathbf{R}^{m} \in \mathscr{M}$ with $\Theta_{\mathbf{C}^{(0)}}(0)=\alpha$ are such that $\mathbf{C}_{0}^{(0)}$ satisfies $\ddagger \ddagger$. Then, for each $M \in \mathscr{M}, \operatorname{sing}_{\alpha} M$ is countably $m$-rectifiable and there is an open $V_{\alpha} \supset \operatorname{sing}_{\alpha} M$ such that $V_{\alpha} \cap$ $\left\{X \in U_{M}: \Theta_{M}(X) \geq \alpha\right\}$ has locally finite $\mathscr{H}^{m}$-measure in $V_{\alpha}$.

Remark. We show in (2.1) that, if $m$ is as in (1.15), the set of possible densities $\boldsymbol{\theta}_{\mathbf{C}}(0)$ corresponding to cylindrical tangent cones $\mathbf{C}=\mathbf{C}_{0} \times \mathbf{R}^{m} \in$ $\mathscr{M}$ is a discrete set, so this result clearly does imply Theorem 2 .

An important point is that the troublesome integrability hypothesis $\ddagger \ddagger$ is automatically satisfied if $m=n-1$ or $m=n-2$. For example if $m=n-2$, then any cylindrical cone $\mathbf{C}=\mathbf{C}_{0} \times \mathbf{R}^{m} \in \mathscr{M}$ has twodimensional cross section $\mathbf{C}_{0}$, so $\mathbf{C}_{0}$ must consist of a finite union of two-dimensional planes $\bigcup_{j=1}^{N} P_{j}$, where each $P_{j}$ passes through the origin and $P_{j} \cap P_{j}=\{0\}$ for $i \neq j$, since the only smooth connected compact embedded one-dimensional minimal submanifolds of $S^{n+k-1}$ are the finite unions of pairwise disjoint great circles. Of course then the integrability condition is trivially satisfied, because the Jacobi field opeator for the plane $P_{j}$ is just the ordinary Laplacian acting on sections of the normal bundle over $P_{j}$. Since such a plane $P_{j} \subset \mathbf{R}^{n+k}$ has an orthonormal basis of constant normal vectors $\eta_{1}^{(j)}, \cdots, \eta_{n+k-2}^{(j)}$, this then means that the Jacobi field operator $\mathscr{L}_{\mathbf{C}_{0}}$ on $P_{j}$ is just the ordinary Laplacian acting on the components relative to these constant basis vectors. Thus the homogeneous degree one solutions of $\mathscr{L}_{\mathrm{C}_{0}} v=0$ on $P_{j}$ are precisely the restrictions to $P_{j}$ of functions of the form $\sum_{i=1}^{l+k-2} l_{i}^{(j)} \eta_{i}^{(j)}$, where the $l_{i}^{(j)}$ are arbitrary linear functions of $x=\left(x^{1}, \cdots, x^{l+k}\right)$. Any such solution is easily seen to be generated (as in Example 1.13) by a family $\mathbf{C}_{t}=\bigcup_{j=1}^{N} P_{j}^{(t)}$, where $P_{j}^{(t)}=\left(\exp t A_{j}\right) P_{j}$, with $A_{j}$ a skew-symmetric transformation of $\mathbf{R}^{l+k}$; thus we claim that $\sum_{i=1}^{l+k-2} l_{i}^{(j)} \eta_{i}^{(j)}=\left(A_{j}(x)\right)^{\perp}$ on $P_{j}$ for suitable skew-symmetric $A_{j}: \mathbf{R}^{l+k} \rightarrow \mathbf{R}^{l+k}$. For example $x \rightarrow x^{p} \eta_{q}^{(j)}$ (for any $p \in\{1, \cdots, l+k\}, q \in\{1, \cdots, l+k-2\})$ is given by $\left(A_{j}(x)\right)^{\perp}$ on $P_{j}$, where $A_{j}$ is the skew-symmetric transformation $x \mapsto x_{p} \eta_{q}^{(j)}-x \cdot \eta_{q}^{(j)} e_{p}$, as one checks keeping in mind the fact that $x \cdot \eta_{q}^{(j)} \equiv 0$ on $P_{j}$.

Likewise for $m=n-1$ (when $\mathbf{C}_{0}$ is simply a finite union of rays emanating from the origin), the integrability condition $\ddagger \ddagger$ is easily checked. 
Thus we have the following corollary of Theorem $2^{\prime}$.

Theorem 3. If $m$ is as in (1.15) and either $m=n-2$ or $m=n-1$, then for each $M \in \mathscr{M}$, $\operatorname{sing} M$ is countably m-rectifiable, and $\operatorname{sing}_{\alpha} M$ has locally finite $\mathscr{H}^{m}$-measure for each $\alpha$; in fact there is an open $V_{\alpha} \supset$ sing $_{\alpha} M$ such that $V_{\alpha} \cap\left\{X: \Theta_{M}(X) \geq \alpha\right\}$ has locally finite $\mathscr{H}^{m}$-measure in $V_{\alpha}$.

Notice in particular that the class $\mathscr{T}_{2}$ of the regular sets of $\bmod 2$ minimizing currents in $\mathbf{R}^{n+k}$ (discussed in $\S 1.4$ ) have $m=n-2$ (by [10]) for any $k \geq 2$, and furthermore (by [14]) any cylindrical cone $\mathbf{C}_{0} \times$ $\mathbf{R}^{n-2} \in \mathscr{T}_{2}$ is such that $\mathbf{C}_{0}$ consists of a union of a collection of $j$ pairwise mutually orthogonal 2-planes in $\mathbf{R}^{k+2}$ (any pair of which have only the origin in common), where $2 \leq j \leq k / 2+1$. We therefore also conclude from Theorem 2 the following:

Corollary 1. Suppose $M$ is the interior regular set of a current $T$ which is $\bmod 2$ minimizing in $\mathbf{R}^{n+k}$ so that $T$ is $\bmod 2$ minimizing in the sense described in $\S 1.4$, and $M=\operatorname{reg} T \backslash \operatorname{spt} \partial_{2} T$. Then $\operatorname{sing} M$ $\left(\equiv \bar{M} \backslash\left(M \cup \partial_{2} T\right)\right)$ is countably $(n-2)$-rectifiable. Also, if $S_{j}$ denotes the set of all points $X \in \operatorname{sing} M$ such that $\operatorname{Tan}_{X} M$ contains a cylindrical cone $q\left(\mathbf{C}_{0} \times \mathbf{R}^{n-2}\right)$ with $q \in \mathrm{SO}\left(\mathbf{R}^{n+k}\right)$ and $\mathbf{C}_{0}$ a union of $j$ pairwise mutually orthogonal 2-planes in $\mathbf{R}^{k+2}$, then $\operatorname{dim}\left(\operatorname{sing} M \backslash\left(\bigcup_{2 \leq j \leq k / 2+1} S_{j}\right)\right) \leq n-3$ for $n \geq 4$, sing $M \backslash\left(\bigcup_{2 \leq j \leq k / 2+1} S_{j}\right)$ is discrete for $n=3$, and $S_{j}$ has locally finite $\mathscr{H}^{n-2}$-measure for each $j$.

Remark. Notice that Theorem $2^{\prime}$ actually implies the stronger result that for each $j$ there is an open $V_{j} \supset S_{j}$ such that $\operatorname{sing} M \cap\left\{X \in V_{j}\right.$ : $\left.\Theta_{M}(X) \geq j\right\}$ has locally finite $\mathscr{H}^{n-2}$-measure in $V_{j}$.

Finally we note one case where we can capitalize on the fact that the first alternative of Lemma 1 always fails: This is the case if the following hold: $X_{0} \in \operatorname{sing} M \cap U_{M}$,

$$
\Theta_{M}\left(X_{0}\right)=\mu \equiv \min \left\{\boldsymbol{\Theta}_{\mathbf{C}}(0): \mathbf{C}=\mathbf{C}_{0} \times \mathbf{R}^{m} \in \mathscr{M}\right\},
$$

and $\mathbf{C}^{(0)}=\mathbf{C}_{0}^{(0)} \times \mathbf{R}^{m} \in \operatorname{Tan}_{X_{0}} M$ is such that the singularity 0 of $\mathbf{C}_{0}^{(0)} \cap \bar{B}_{1}$ cannot be perturbed away in $\bar{B}_{1}$ in such a way that the boundary $\Sigma_{0}=$ $\mathbf{C}_{0}^{(0)} \cap S^{n+k-1}$ is held fixed; that is, when

there does not exist a smooth embedded compact l-dimensional manifold-with-boundary $\widetilde{M} \subset \widetilde{B}_{1}$ such that $\partial \widetilde{M}=$ $\Sigma_{0}$. 
Then we have

Theorem 4. Suppose $m$ is as in (1.15). Suppose also that $M \in \mathscr{M}$, $\mathbf{C}^{(0)}=\mathbf{C}_{0}^{(0)} \times \mathbf{R}^{m} \in \operatorname{Tan}_{X_{0}} M$ satisfies $\ddagger$, that $\Theta_{\mathbf{C}^{(0)}}(0)=\mu, \mu$ as in (1.16), and that (1.17) holds. Then there is a $\rho>0$ such that $B_{\rho}\left(X_{0}\right) \cap \operatorname{sing} M$ is an embedded m-dimensional $C^{1, \alpha}$ manifold for some $\alpha=\alpha(\mathscr{M}) \in(0,1)$.

Notice that both $\ddagger \ddagger$ and (1.17) trivially hold in case $m=n-1$ and $\mathbf{C}_{0}$ consists of an odd number $p$ of rays emanating from $\mathbf{C}$. Thus we arrive at

Corollary 2. Suppose the $m$ of $(1.15)$ is equal to $(n-1)$. If $M \in$ $\mathscr{M}, \mathbf{C}^{(0)}=\mathbf{C}_{0}^{(0)} \times \mathbf{R} \in \operatorname{Tan}_{X_{0}} M$ with $\mathbf{C}_{0}^{(0)}$ consisting of an odd number of rays emanating from 0 , and (1.16) holds, then there is $\rho>0$ such that $\operatorname{sing} M \cap B_{\rho}\left(X_{0}\right)$ is a properly embedded $(n-1)$-dimensional $C^{1, \alpha}$ manifold.

We also have the following corollary, which will be proved in $\S 6$.

Corollary 3. If $\mathbf{V}$ is an n-dimensional stationary integral varifold in some open set $U \subset \mathbf{R}^{n+k}$ and $X_{0} \in U$ with $1<\Theta_{\mathbf{V}}\left(X_{0}\right)<2$, then $\operatorname{sing} \mathbf{V} \cap B_{\rho}\left(X_{0}\right)$ is the union of an embedded $(n-1)$-dimensional $C^{1, \alpha}$ manifold and a closed set of dimension $\leq n-2$. If $n=2$ we have the more precise conclusion that there is $\rho>0$ such that either $\operatorname{sing} \mathrm{V} \cap B_{\rho}\left(X_{0}\right)$ is a properly embedded $C^{1, \alpha}$ Jordan arc with endpoints in $\partial B_{\rho}\left(X_{0}\right)$ or else is a finite union of properly embedded locally $C^{1, \alpha}$ Jordan arcs of finite length, each with one endpoint at $X_{0}$ and one endpoint in $\partial B_{\rho}\left(X_{0}\right)$.

Remark. By a properly embedded locally $C^{1, \alpha}$ Jordan arc $\Gamma$ we mean a homeomorphic image of $[0,1]$ such that for each compact subarc $K \subset \Gamma$ not containing either of the endpoints of $\Gamma$ there is a $C^{1, \alpha}$ diffeomorphism of $[0,1]$ onto $K$.

\section{Technical preliminaries}

First we recall some important facts about the set of compact embedded minimal submanifolds $\Sigma$ of dimension $l-1 \quad(l \geq 2)$ which are $C^{2}$-close to $\Sigma_{0}, \Sigma_{0}=\mathbf{C}_{0}^{(0)} \cap S^{l+k-1}$ as in $\S 1$. For each $\varepsilon_{0}>0$ let

$$
\begin{aligned}
\mathscr{G}_{\varepsilon_{0}}^{(1)}= & \left\{\operatorname{graph} u \cap S^{l+k-1}: u \in C^{2}\left(\mathbf{C}_{0}^{(0)} ;\left(\mathbf{C}_{0}^{(0)}\right)^{\perp}\right),\right. \\
& \left.u(r \omega)=r u(\omega) \forall r>0, \omega \in \Sigma_{0},|u|_{C^{2}\left(\Sigma_{0}\right)}<\varepsilon_{0}\right\},
\end{aligned}
$$




$$
\begin{aligned}
\mathscr{V}= & \text { the compact embedded }(l-1) \text {-dimensional minimal } \\
& \text { submanifolds } \in S^{l+k-1} .
\end{aligned}
$$

Notice that then, with $\mathscr{Q}_{\mathbf{C}_{0}}^{(0)}$ the Euler-Lagrange operator of the area functional as in $\S 1$,

$$
\begin{aligned}
& \mathscr{V} \cap \mathscr{G}_{\varepsilon_{0}}^{(1)}=\left\{\operatorname{graph} u \cap S^{l+k-1}:\right. \\
& u \in C^{2}\left(\mathbf{C}_{0}^{(0)} ;\left(\mathbf{C}_{0}^{(0)}\right)^{\perp}\right), u(r \omega)=r u(\omega),|u|_{C^{2}\left(\Sigma_{0}\right)}<\varepsilon_{0}, \\
& \left.\mathscr{Q}_{\mathbf{C}_{0}^{(0)}} u=0\right\} .
\end{aligned}
$$

Recall also (see, e.g., the discussion of [18]) that, for $\varepsilon_{0}$ sufficiently small (depending on $\mathbf{C}_{0}^{(0)}$ ), $\mathscr{V} \cap \mathscr{G}_{\varepsilon_{0}}^{(1)}$ is a real-analytic variety in $\mathscr{G}_{\varepsilon_{0}}^{(1)}$. Hence in particular for suitably small $\varepsilon_{0}>0 \mathscr{H}^{l-1}(\Sigma)=\mathscr{H}^{l-1}\left(\Sigma_{0}\right) \quad \forall \Sigma \in \mathscr{V} \cap \mathscr{G}_{\varepsilon_{0}}^{(1)}$, and

$$
\boldsymbol{\Theta}_{\widetilde{\mathbf{C}}}(0)=\boldsymbol{\Theta}_{\mathbf{C}^{(0)}}(0) \quad \forall \widetilde{\mathbf{C}} \in \tilde{\mathscr{C}}_{\varepsilon_{0}}\left(\mathbf{C}^{(0)}\right),
$$

where $\varepsilon_{0}=\varepsilon_{0}\left(\mathbf{C}^{(0)}\right)>0$. Also, since $\mathscr{L}_{\mathbf{C}^{(0)}}$ is the linearization of $\mathscr{Q}_{\mathbf{C}^{(0)}}$, we have $\delta=\delta\left(\varepsilon, \mathbf{C}^{(0)}\right)$ with $\delta\left(\varepsilon, \mathbf{C}^{(0)}\right) \downarrow 0$ as $\varepsilon \downarrow 0$ such that if $\varepsilon \leq \varepsilon_{0}$ then

$$
\mathbf{C} \in \widetilde{\mathscr{C}}_{\varepsilon}\left(\mathbf{C}^{(0)}\right) \Rightarrow \mathbf{C} \cap\left\{(x, y) \in B_{1}:|x|>\delta|y|\right\}=\operatorname{graph} \psi,
$$

where $\psi \in C^{2}\left(\Omega ;\left(\mathbf{C}^{(0)}\right)^{\perp}\right)$ with $\left\{(x, y) \in \mathbf{C}^{(0)}:|x|>\delta|y|\right\} \subset \Omega \subset \mathbf{C}^{(0)}$, and by Definition 1.12(ii),

$$
\psi(x, y)=\sum_{i=1}^{l+k} y \cdot \eta_{i} e_{i}^{\perp}+r \varphi(\omega)+R(x, y), \quad r=|x|, \omega=|x|^{-1} x
$$

for some $\eta_{i} \in \mathbf{R}^{m}, \varphi \in C^{2}\left(\Sigma_{0} ;\left(\mathbf{C}_{0}^{(0)}\right)^{\perp}\right)$, with $\left|\eta_{i}\right|<C \varepsilon$, sup $|\varphi| \leq C \varepsilon$, $\mathscr{L}_{\mathbf{C}_{0}^{(0)}}(r \varphi(\omega))=0, r^{-1}|R|+|\nabla R| \leq C \varepsilon^{2}$ for $r \geq \delta, C=C\left(\mathbf{C}^{(0)}\right)$. Of course subject to the integrability condition $\ddagger \ddagger$ the possible $r \varphi(\omega)$ which appear here account for all homogeneous degree 1 solutions $v=r \varphi(\omega)$ of $\mathscr{L}_{\mathbf{C}^{(0)}} v=0$, and the additional terms $\sum_{j=1}^{l+k} \eta_{j} \cdot y e_{j}^{\perp}$ are generated as in Example 1.13 by rotations $\exp (t A), A \in \mathscr{S}, \mathscr{S}$ as in Definition 1.12(ii). Using (1.8), it then directly follows that if (łł) holds, if $M_{j} \in \mathscr{N}_{\varepsilon_{j}}\left(\mathbf{C}^{(0)}\right)$, if $\mathbf{C}_{j} \in \mathscr{C}_{\varepsilon_{j}}\left(\mathbf{C}^{(0)}\right)$, where $\varepsilon_{j} \downarrow 0$, if $\int_{M_{j} \cap B_{1}} \operatorname{dist}^{2}\left(X, \mathbf{C}_{j}\right) \leq \beta_{j}^{2}$, where $\beta_{j} \downarrow 0$, and if $\eta_{q} \in \mathbf{R}^{m}, q=1, \cdots, l+k, \varphi \in C^{2}\left(\Sigma_{0} ;\left(\mathbf{C}^{(0)}\right)^{\perp}\right)$ with $\mathscr{L}_{\mathbf{C}^{(0)}}(r \varphi)=$ 0 and $\left|\eta_{q}\right|+\sup |\varphi| \leq K$, then for some subsequence $\{i\} \subset\{j\}$ we can 
find $\widetilde{\mathbf{C}}_{i} \in \mathscr{C}_{\gamma \beta_{i}}\left(\mathbf{C}_{i}\right) \quad\left(\gamma=\gamma\left(\mathbf{C}^{(0)}, K\right)\right.$ fixed $)$ and $\delta_{i}, \tau_{i} \downarrow 0$ such that

$$
M_{j} \cap\left\{(x, y) \in B_{1}:|x|>\delta_{i}\right\}=\operatorname{graph} u_{i}=\operatorname{graph} \tilde{u}_{i},
$$

where $u_{i} \in C^{2}\left(\Omega_{i} ; \mathbf{C}_{i}^{\perp}\right), \tilde{u}_{i} \in C^{2}\left(\widetilde{\Omega}_{i} ; \widetilde{\mathbf{C}}_{i}^{\perp}\right)$ with $\left\{X=(x, y) \in \mathbf{C}_{i}:|x|>\right.$ $\left.\tau_{i}\right\} \subset \Omega_{i} \subset \mathbf{C}_{i},\left\{X=(x, y) \in \widetilde{\mathbf{C}}_{i}:|x|>\tau_{i}\right\} \subset \widetilde{\Omega}_{i} \subset \widetilde{\mathbf{C}}_{i}$ and

$$
\begin{gathered}
\beta_{i}^{-1}\left(u_{i}\left((x, y)+\psi_{i}(x, y)\right)-\tilde{u}_{i}\left((x, y)+\tilde{\psi}_{i}(x, y)\right)\right) \\
=\sum_{j=1}^{l+k} y \cdot \eta_{j} e_{j}^{\perp}+r \varphi(\omega)+R_{i}
\end{gathered}
$$

on $\left\{(x, y) \in \mathbf{C}^{(0)} \cap B_{3 / 4}:|x|>\tau_{i}\right\}$, where $\sup \left|R_{i}\right| \rightarrow 0$ and $\psi_{i}, \tilde{\psi}_{i}$ correspond to $\psi$ of (2.2) with $\mathbf{C}_{i}, \widetilde{\mathbf{C}}_{i}$ respectively in place of $\mathbf{C}$.

In the following lemma, we let $\mathscr{K}$ denote the set of cones $\mathbf{C} \in \mathscr{M}$ with vertex at 0 ; thus $\mathbf{C} \in \mathscr{K}$ means $\mathbf{C} \in \mathscr{M}$ and $\eta_{0, \lambda} \mathbf{C}=\mathbf{C}$ for each $\lambda>0$. Then we have:

2.4. Lemma. Let $K>\alpha>1$ be given. There are $\delta:(0,1) \rightarrow(0,1)$ and $R:(0,1) \rightarrow(2, \infty)$ (depending on $K, \mathscr{M})$ such that if $\varepsilon \in(0,1)$, $M \in \mathscr{M}, U_{M} \supset B_{R(\varepsilon)}(0), 0 \in \operatorname{sing} M$, and

$$
\mathscr{C}^{n}\left(M \cap B_{R(\varepsilon)}\right) / \omega_{n} R(\varepsilon)^{n}-\alpha<\delta(\varepsilon),
$$

then $X_{1} \in\left\{X \in B_{1}: \Theta_{M}(X) \geq \alpha\right\}$ implies the following hold:

(i) $0 \leq\left(\omega_{n} \rho^{n}\right)^{-1} \mathscr{H}^{n}\left(M \cap B_{\rho}\left(X_{1}\right)\right)-\alpha<\varepsilon^{2}$ for all $\rho \leq R(\varepsilon)-1$.

(ii) There is $\mathbf{C} \in \mathscr{K}$ such that $\left|\Theta_{\mathbf{C}}(0)-\alpha\right| \leq \varepsilon^{2}$ and

$$
\int_{\eta_{X_{1}, \rho} M \cap B_{1}} \operatorname{dist}^{2}(X, \mathbf{C})<\varepsilon^{2}, \quad \rho \in(0,1],
$$

and, if $m$ is as in (1.15), for all $\rho \in(0,1]$

(iii) either there is a cylindrical cone $\mathbf{C}=q\left(\mathbf{C}_{0} \times \mathbf{R}^{m}\right) \in \mathscr{K}$ with $\left|\Theta_{\mathbf{C}}(0)-\alpha\right| \leq \varepsilon^{2}$ and

or

$$
\int_{\eta_{X_{1}, \rho} M \cap B_{1}} \operatorname{dist}^{2}(X, \mathbf{C})<\varepsilon^{2},
$$

$$
\left\{X \in \bar{B}_{1}: \Theta_{\eta_{X_{1}, \rho} M}(X) \geq \alpha\right\} \subset\{X: \operatorname{dist}(X, H)<\varepsilon\}
$$

for some $(m-1)$-dimensional subspace $H$ of $\mathbf{R}^{n+k}$. (We emphasize that $\mathrm{C}$ and $H$ depend on $\rho$ here.) 
Proof. (i) and (ii) are both easy consequences of (1.5) and the compactness $1.3(\mathrm{~b})$ of $\mathscr{M}$. (See, e.g., [1] or [19] for similar arguments.)

To prove (iii) we note the contrary implies that for some fixed $\varepsilon \in(0,1)$ there are sequences $\rho_{j} \in(0,1], \alpha_{j}, \delta_{j} \downarrow 0, R_{j} \uparrow \infty, M_{j} \in \mathscr{M}$ with $1+\alpha_{0} \leq \alpha_{j} \leq K \quad\left(\alpha_{0}\right.$ as in (1.7)) and $U_{M_{j}} \supset B_{R_{j}}$, and a sequence $X_{j} \in B_{1}$ with $\Theta_{M_{j}}\left(X_{j}\right) \geq \alpha_{j}$ such that, with $\widetilde{M}_{j}=\eta_{X_{j}, \rho_{j}} M_{j}$,

$$
\begin{gathered}
0 \leq \mathscr{H}^{n}\left(\widetilde{M}_{j} \cap B_{R_{j}}\right) / \omega_{n} R_{j}^{n}-\alpha_{j}<\delta_{j}, \\
\int_{\widetilde{M}_{j} \cap B_{1}} \operatorname{dist}^{2}(X, \mathbf{C}) \geq \varepsilon^{2} \quad \text { or } \quad\left|\Theta_{\mathbf{C}}(0)-\alpha_{j}\right| \geq \varepsilon^{2},
\end{gathered}
$$

for every cylindrical cone $\mathbf{C}=q\left(\mathbf{C}_{0} \times \mathbf{R}^{m}\right) \in \mathscr{K}$, and

$$
\left\{X \in \bar{B}_{1}: \Theta_{\widetilde{M}_{j}}(X) \geq \alpha_{j}\right\} \not \subset\{X: \operatorname{dist}(X, H)<\varepsilon\}
$$

for all $(m-1)$-dimensional subspaces $H \subset \mathbf{R}^{n+k}$. By (iii) there are corresponding cones $\mathbf{C}^{(j)} \in \mathscr{K}$ such that

(4) $\int_{\widetilde{M}_{j} \cap B_{2}} \operatorname{dist}^{2}\left(X, \mathbf{C}^{(j)}\right) \rightarrow 0 \quad$ and $\quad \Theta_{\mathbf{C}^{(j)}}(0)-\alpha_{j} \rightarrow 0 \quad$ as $j \rightarrow \infty$,

and by the compactness property $1.3(\mathrm{~b})$ there are subsequences (still denoted $\widetilde{M}_{j}, \mathbf{C}^{(j)}$ ) and $\mathbf{C}_{*} \in \mathscr{K}$ such that

$$
\widetilde{M}_{j} \rightarrow \mathbf{C}_{*} \quad \text { in } B_{2}, \quad \mathbf{C}^{(j)} \rightarrow \mathbf{C}_{*} \quad \text { in } \mathbf{R}^{n+k}
$$

in the sense of 1.3(b). Also by (4) and (5) we obtain

$$
\alpha_{j}, \boldsymbol{\Theta}_{\widetilde{M}_{j}}(0) \rightarrow \boldsymbol{\Theta}_{\mathbf{C}_{*}}(0)
$$

and

$$
\int_{\widetilde{M}_{j} \cap B_{2}} \operatorname{dist}^{2}\left(X, \mathbf{C}_{*}\right) \rightarrow 0,
$$

and hence, by (2), $\mathbf{C}_{*}$ does not have the form $q\left(\mathbf{C}_{0} \times \mathbf{R}^{m}\right) \in \mathscr{K}$. Then (see the discussion preceding (1.10)) we have

$$
\left\{X \in \overline{\mathbf{C}}_{*}: \boldsymbol{\Theta}_{\mathbf{C}_{*}}(X) \geq \boldsymbol{\Theta}_{\mathbf{C}_{*}}(0)\right\} \subset H
$$

for some $(m-1)$-dimensional subspace $H \subset \mathbf{R}^{n+k}$. Then by (5), (6) and the upper semicontinuity of densities (in the sense that $\Theta_{\mathbf{C}_{*}}(Y) \geq$ $\limsup \Theta_{\widetilde{M}_{j}}\left(Y_{j}\right)$ for any sequence $Y_{j} \rightarrow Y$ ) we get

$$
\left\{X \in B_{1}: \boldsymbol{\Theta}_{\widetilde{M}_{j}}(X) \geq \alpha_{j}\right\} \subset\{X: \operatorname{dist}(X, H)<\varepsilon\}
$$


for all sufficiently large $j$, thus contradicting (3). Hence Lemma 2.4 is proved.

Subsequently, we use the first variation formula (1.1) in a number of places; in particular we shall need the following direct consequence of this formula. (For general discussion of first variation, see [1], [19].) Here let $M \in \mathscr{M}$ with $U_{M} \supset B_{1}$, and for each $X \in M$ let $\left(g^{i j}\right)$ be the matrix of the orthogonal projection of $\mathbf{R}^{n+k}$ onto the tangent space $T_{X} M$ of $M$ at $X$; then $\left(g^{i j}\right)$ is a symmetric $(n+k) \times(n+k)$ matrix with $n$ eigenvalues equal to 1 and $k$ eigenvalues equal to zero, and $\sum_{q=1}^{n+k} g^{i q} g^{j q}=g^{i j}$. Notice that thus the gradient operator $\nabla^{M}$ appearing in (1.1) is given at a point $X=(x, y) \in M$ by

$$
e_{j} \cdot \nabla^{M}=\sum_{i=1}^{l+k} g^{i j}(x, y) \frac{\partial}{\partial x^{i}}+\sum_{i=1}^{m} g^{l+k+i, j} \frac{\partial}{\partial y^{i}}, \quad j=1, \cdots, n+k .
$$

Then by substituting $\Phi(X)=\psi^{2}(x, y)(x, 0), X=(x, y) \in \mathbf{R}^{l+k} \times \mathbf{R}^{m}=$ $\mathbf{R}^{n+k}$, into (1.1) directly, we get the identity

$$
\begin{aligned}
\int_{M} & \left(l+\sum_{j=1}^{m}\left|e_{l+k+j}^{\perp}\right|^{2}\right) \psi^{2} \\
= & -\int_{M}\left(\sum_{i, j=1}^{l+k} g^{i j} x^{i} D_{x^{j}} \psi^{2}-2 \psi \sum_{k=1}^{l+k} \sum_{j=1}^{m}\left(\delta_{i l+k+j}-g^{i, l+k+j}\right) x^{i} D_{y^{j}} \psi\right) \\
& \leq \int_{M}\left(-\sum_{i, j=1}^{l+k} g^{i j} x^{i} D_{x^{j}} \psi^{2}+2\left|(x, 0)^{\perp}\right||\psi|\left(\sum_{j=1}^{m}\left|e_{l+k+j}^{\perp}\right|^{2}\right)^{1 / 2}\left|D_{y} \psi\right|\right.
\end{aligned}
$$

for any $\psi \in C_{c}^{\infty}\left(B_{1}\right)$, where $e_{l+k+j}^{\perp}$ is the orthogonal projection of $e_{l+k+j}$ onto $\left(T_{(x, y)} M\right)^{\perp}$ and $(x, 0)^{\perp}$ is the orthogonal projection of $(x, 0)$ onto $\left(T_{(x, y)} M\right)^{\perp}$. Thus

$$
\left|e_{l+k+j}^{\perp}\right|^{2}=1-g^{l+k+j, l+k+j}
$$

and

$$
\left|(x, 0)^{\perp}\right|^{2}=\sum_{i, j=1}^{l+k}\left(\delta_{i j}-g^{i j}\right) x^{i} x^{j}=\sum_{q=1}^{n+k}\left(\sum_{i=1}^{l+k}\left(\delta_{i q}-g^{i q}\right) x^{i}\right)^{2},
$$

since $g^{i j}=\sum_{q=1}^{n+k} g^{i q} g^{j q}$ for every $i, j=1, \cdots, n+k$. Notice that, by using the above inequality in combination with the Cauchy inequality, we 
obtain

$$
\begin{aligned}
\int_{M}\left(l+\frac{1}{2} \sum_{j=1}^{m} \mid\right. & \left.\left.e_{l+k+j}^{\perp}\right|^{2}\right) \psi^{2} \\
& \leq \int_{M}\left(-\sum_{i, j=1}^{l+k} g^{i j} x^{i} D_{x^{j}} \psi^{2}+2\left|(x, 0)^{\perp}\right|^{2}\left|D_{y} \psi\right|^{2}\right) .
\end{aligned}
$$

We now want to make some observations about the extent to which we can initially take $M \in \mathscr{N}_{\varepsilon}\left(\mathbf{C}^{(0)}\right)$ to be represented graphically (via a normal section over a region in a given cone $\left.\mathbf{C} \in \mathscr{C}_{\varepsilon}\left(\mathbf{C}^{(0)}\right)\right)$, and some initial $L^{2}$ estimates for such normal sections.

2.6. Lemma. Suppose $\gamma, \beta, \tau \in(0,1)$ are arbitrary with $\tau \leq$ $(1-\gamma) / 10$. There is $\varepsilon_{0}=\varepsilon_{0}\left(\mathbf{C}^{(0)}, \mathscr{M}, \gamma, \beta, \tau\right) \in(0,1]$ such that if $\mathbf{C}=\mathbf{C}_{0} \times \mathbf{R}^{m} \in \mathscr{C}_{\varepsilon_{0}}\left(\mathbf{C}^{(0)}\right)$ and $M \in \mathscr{N}_{\varepsilon_{0}}\left(\mathbf{C}^{(0)}\right)$, then there is an open $U \subset \mathrm{C} \cap B_{1}$ with the properties

$$
\begin{gathered}
(x, y) \in U \Rightarrow(\tilde{x}, y) \in U \text { whenever }(\tilde{x}, y) \in \mathbf{C} \text { with }|\tilde{x}|=|x|, \\
\left\{(x, y) \in \mathbf{C} \cap B_{\gamma}:|x|>\tau\right\} \subset U,
\end{gathered}
$$

and such that there is a $u \in C^{2}\left(U ; \mathbf{C}^{\perp}\right)$ with $M \cap B_{\gamma} \cap\{(x, y):|x|>\tau\} \subset \operatorname{graph} u \subset M, \quad \sup r^{-1}|u|+\sup |\nabla u| \leq \beta$, and

$\int_{M \cap B_{\gamma} \backslash \text { graph } u} r^{2}+\int_{U \cap B_{\gamma}} r^{2}|\nabla u|^{2} \leq C \int_{M \cap B_{1}} d^{2}, \quad C=C\left(\mathbf{C}^{(0)}, \mathscr{M}, \gamma, \beta\right) ;$ note in particular that $C$ is independent of $\tau$. Here $r(x, y)=|x|$, $d(x, y)=\operatorname{dist}((x, y), \mathbf{C}),(x, y) \in \mathbf{R}^{l+k} \times \mathbf{R}^{m}$.

Remark. Notice that by using the standard elliptic $L^{2}$ estimates for $u$ we conclude from the above that

Hausdorff distance $\left(M \cap B_{1 / 4}(e), \mathrm{C} \cap B_{1 / 4}(e)\right) \leq C\left(\int_{M \cap B_{1}} d^{2}\right)^{1 / 2}$, whenever $e \in \mathbf{C}_{0} \times\{0\}$ with $|e| \leq 1 / 2$.

Proof. Consider $M \in \mathscr{N}_{\varepsilon_{0}}\left(\mathbf{C}^{(0)}\right)$ and $\mathbf{C}=\mathbf{C}_{0} \times \mathbf{R}^{m} \in \mathscr{C}_{\varepsilon_{0}}\left(\mathbf{C}^{(0)}\right)$, where for the moment $\varepsilon_{0}>0$ is arbitrary. For each $\kappa, \rho \in(0,1], \zeta \in \mathbf{R}^{m}$, let $T_{\rho, \kappa}(\zeta)$ be the torus defined by

(1) $T_{\rho, \kappa}(\zeta)=\left\{(x, y) \in \mathbf{R}^{l+k} \times \mathbf{R}^{m}:(|x|-\rho)^{2}+|y-\zeta|^{2}<\kappa^{2}(1-\gamma)^{2} \rho^{2} / 4\right\}$. 
Let $U$ be the union of all $T_{|\xi|, 1 / 2}(\zeta) \cap \mathbf{C}$ over all $(\xi, \zeta) \in \mathbf{C} \cap B_{\gamma}$ such that there exists $u_{|\xi|, \zeta} \in C^{2}\left(T_{|\xi|, 3 / 4}(\zeta) ; \mathbf{C}^{\perp}\right)$ with

$$
M \cap T_{|\xi|, 1 / 2}(\zeta) \subset \operatorname{graph} u_{|\xi|, \zeta} \subset M
$$

and

$$
|\xi|^{-1} \sup _{\mathbf{C} \cap T_{|\xi|, 3 / 4}(\zeta)}\left|u_{|\xi|, \zeta}\right|+\sup _{\mathbf{C} \cap T_{|\xi|, 3 / 4}(\zeta)}\left|\nabla u_{|\xi|, \zeta}\right|<\beta / 2 .
$$

Let $u \in C^{2}\left(U ; \mathbf{C}^{\perp}\right)$ be defined on $T_{|\xi|, 1 / 2}(\zeta) \cap \mathbf{C}$ by

$$
u\left|T_{|\xi|, 1 / 2}(\zeta) \cap \mathrm{C}=u_{|\xi|, \zeta}\right| T_{|\xi|, 1 / 2}(\zeta) \cap \mathrm{C} .
$$

By unique continuation of solutions of $\mathscr{Q}_{\mathbf{C}} u=0$, (4) makes sense as a definition and does give a $C^{2}\left(U ; \mathbf{C}^{\perp}\right)$ function $u$. Notice also that since $M \in \mathscr{N}_{\varepsilon_{0}}\left(\mathbf{C}^{(0)}\right)$ it follows from (1.8) and 1.3(b) (see also the remark following Definition 1.11) that

$$
\left\{(x, y) \in \mathbf{C} \cap B_{\gamma}:|x|>\tau\right\} \subset U
$$

and

$$
M \cap B_{\gamma} \cap\{(x, y):|x|>\tau\} \subset \operatorname{graph} u \subset M
$$

as required, provided that $\varepsilon_{0}=\varepsilon_{0}\left(\tau, \gamma, \beta, \mathbf{C}^{(0)}, \mathscr{M}\right)$ is chosen sufficiently small. Of course by definition we have

$$
\sup _{U}|x|^{-1}|u(x, y)|+\sup _{U}|\nabla u| \leq \beta .
$$

Furthermore, if $(\xi, \zeta) \in \mathbf{C} \cap B_{\gamma} \cap \partial U$, then

$$
\int_{M \cap T_{|\xi|, 1}(\zeta)} d^{2} \geq C^{-1} \beta^{2}|\xi|^{n+2}, \quad C=C\left(\mathbf{C}^{(0)}, \mathscr{M}\right),
$$

because otherwise by (1.8) we would have $u_{|\xi|, \zeta}$ as in (2), (3), thus contradicting the fact that $(\xi, \zeta) \in \mathbf{C} \cap B_{\gamma} \cap \partial U$. (Notice that $\mathbf{C} \cap\{0\} \times \mathbf{R}^{m}=\varnothing$, so $|\xi|>0$ here.) Also, for such $(\xi, \zeta)$, since $|\xi|<\tau<(1-\gamma) / 10$ and $\mathscr{H}^{n}\left(M \cap B_{1}\right) \leq \mathscr{H}^{n}\left(\mathbf{C}^{(0)} \cap B_{1}\right)+1$ by Definition 1.11 , we have by (1.5)

$$
\int_{U \cap B_{10|\xi|}(0, \zeta)} r^{2} \leq C|\xi|^{n+2}, \quad C=C\left(\mathbf{C}^{(0)}\right) .
$$

But also $|\nabla u(x, y)| \leq \beta$ for $(x, y) \in U$ by (5), so (7) implies

$$
\int_{U \cap B_{10|\xi|}(0, \zeta)} r^{2}|\nabla u|^{2} \leq C \beta^{2}|\xi|^{n+2}
$$


with $C=C\left(\mathbf{C}^{(0)}, \mathscr{M}, \gamma\right)$ and then from (6) and (8) it follows that (9) $\int_{U \cap B_{10|\xi|}(0, \zeta)} r^{2}|\nabla u|^{2} \leq C \int_{M \cap T_{|\xi|, 1}(\zeta)} d^{2}, \quad C=C\left(\mathbf{C}^{(0)}, \mathscr{M}, \gamma, \beta\right)$, whenever $(\xi, \zeta) \in \mathbf{C} \cap B_{\gamma} \cap \partial U$. Since

$$
\begin{aligned}
&\{(x, y)\left.\in U \cap B_{y}: \operatorname{dist}\left((x, y), B_{\gamma} \cap \partial U\right)<\frac{1}{2}|x|\right\} \\
& \subset \bigcup_{\left\{(x, y) \in \mathbf{C} \cap \partial U \cap B_{y}\right\}} B_{2|x|}(0, y)
\end{aligned}
$$

and

$$
B_{2\left|\xi_{1}\right|}\left(0, \zeta_{1}\right) \cap B_{2\left|\xi_{2}\right|}\left(0, \zeta_{2}\right)=\varnothing \Rightarrow T_{\left|\xi_{1}\right|, 1}\left(\zeta_{1}\right) \cap T_{\left|\xi_{2}\right|, 1}\left(\zeta_{2}\right)=\varnothing,
$$

by virtue of the " 5 -times covering lemma" ([9] or [19]) and (9) we obtain

$$
\int_{\left\{(x, y) \in U \cap B_{y}: \operatorname{dist}\left((x, y), B_{y} \cap \partial U\right) \leq \frac{|x|}{2}\right\}} r^{2}|\nabla u|^{2} \leq C \int_{M \cap B_{1}} d^{2} .
$$

But, for any $(x, y) \in B_{y} \cap U$ with $\operatorname{dist}\left((x, y), B_{y} \cap \partial U\right) \geq \frac{1}{2}|x|$, from (3) and the standard $L^{2}$ elliptic estimates ([12]) it follows that

$$
\int_{\mathbf{C} \cap B_{\rho / 2}(x, y)} r^{2}|\nabla u|^{2} \leq C \int_{\mathbf{C} \cap B_{\rho}(x, y)}|u|^{2}, \quad \rho \in\left(0, \frac{(1-\gamma)|x|}{2}\right) .
$$

By covering the set

$$
\left\{(x, y) \in U \cap B_{\gamma}: \operatorname{dist}\left((x, y), B_{\gamma} \cap \partial U\right) \geq \frac{1}{2}|x|\right\}
$$

by a countable collection of such balls $\left\{B_{\rho / 2}(x, y)\right\}$ in such a way that the corresponding collection $\left\{B_{\rho}(x, y)\right\}$ can be divided into $C=C\left(\mathbf{C}^{(0)}\right)$ pairwise disjoint subcollections, we thus conclude that

$$
\begin{aligned}
\int_{\left\{(x, y) \in U \cap B_{\gamma}: \operatorname{dist}\left((x, y), B_{\gamma} \cap \partial U\right) \geq \frac{|x|}{2}\right\}} r^{2}|\nabla u|^{2} & \leq C \int_{U \cap B_{1}}|u|^{2} \\
& \leq C \int_{M \cap B_{1}} d^{2},
\end{aligned}
$$

where $C=C\left(\mathbf{C}^{(0)}, \gamma\right)$.

Now again consider $(\xi, \eta) \in \mathbf{C} \cap B_{\gamma} \cap \partial U$. Then (6) and (7) imply that

$$
\int_{M \cap B_{10|\xi|}(0, \zeta)} r^{2} \leq C \int_{M \cap T_{|\xi|, 1}(\zeta)} d^{2}, \quad C=C\left(\mathbf{C}^{(0)}, \mathscr{M}, \beta\right)
$$


Note that, by (2), the union of $M \cap B_{2|\xi|}(0, \zeta)$ over $(\xi, \zeta) \in \mathbf{C} \cap B_{\gamma} \cap \partial U$ contains $M \cap B_{\gamma} \backslash \operatorname{graph} u$. Hence using the 5-times covering lemma as before, we conclude that

$$
\int_{M \cap B_{\gamma} \backslash G} r^{2} \leq C \int_{M \cap B_{1}} d^{2}
$$

The proof is now completed by adding (10), (11), and (12).

In the proof of the decomposition theorem (Theorem 1), we shall need the following covering lemma, in which we use the notation that if $Q=$ $\xi+[-\rho, \rho]^{m}$ is a cube in $\mathbf{R}^{m}$, then

$$
e(Q)=\text { the edge length of } Q=2 \rho,
$$

and

$$
Q^{(N)}=\text { the } N \text {-times enlargement of } Q \equiv \xi+[-N \rho, N \rho]^{m} .
$$

Also, if $\mathscr{Q}$ is a collection of cubes, then

$$
\mathscr{Q}^{(N)}=\left\{Q^{(N)}: Q \in \mathscr{Q}\right\} .
$$

All cubes considered here are closed and will always have edges parallel to the coordinate axes, unless otherwise explicitly indicated.

2.7. Lemma. Let $\delta=1 / 2^{N}$ for some integer $N \geq 4$, let $Q_{0} \equiv$ $\left[-\frac{1}{2}, \frac{1}{2}\right]^{m}$ be the unit cube in $\mathbf{R}^{m}$, let $F \subset Q_{0}$ be arbitrary, and let $\mathscr{Q}$ be any collection of cubes of edge length $\leq \frac{1}{8}$ such that $F \subset \cup_{Q \in \mathscr{Q}} Q$, and

$$
Q \in \mathscr{Q} \Rightarrow \exists \text { a cube } Q_{1} \subset Q \text { with } e\left(Q_{1}\right) \geq 8 \delta e(Q) \text { and } Q_{1} \cap F=\varnothing \text {. }
$$

Then there is a collection $\widetilde{\mathscr{Q}}$ of subcubes of $Q_{0}$ such that

$$
F \subset \bigcup_{\widetilde{Q} \in \widetilde{Q}} \tilde{Q}, \sum_{\widetilde{Q} \in \widetilde{Q}} e(\widetilde{Q})^{m} \leq 1-\delta^{2 m},
$$

and

(ii) $\widetilde{Q} \in \widetilde{Q} \Rightarrow \exists Q \in \mathscr{Q}$ with $\widetilde{Q} \subset Q^{(5)}$ and $\frac{\delta}{4} e(Q) \leq e(\widetilde{Q})<e(Q)$.

Proof. For each $j=1,2, \cdots$ let $\mathscr{C}_{j}$ be the collection of $2^{m j}$ congruent subcubes of edge length $2^{-j}$ obtained by repeated subdivision of the unit cube $\left[\frac{1}{2}, \frac{1}{2}\right]^{m}$. Let $\mathscr{P}_{0}=\varnothing$ and for each $j \geq 1$ let $\mathscr{P}_{j}$ be the collection of cubes in $\mathscr{C}_{j}$ which intersect at least one of the cubes in $\mathscr{Q}_{j}$, where

$$
\mathscr{Q}_{j}=\left\{Q \in \mathscr{Q}: 1 / 2^{j+1}<e(Q) \leq 1 / 2^{j}\right\}, \quad j=1,2, \cdots .
$$


Notice that $P \in \mathscr{P}_{j} \Rightarrow \exists P_{1} \in \mathscr{P}_{j}$ with

$$
P_{1} \cap P \neq \varnothing \text { and } P_{1} \supset Q_{1},
$$

where $Q_{1}$ is a cube contained in some $Q \in \mathscr{Q}_{j}$ such that $F \cap Q_{1}=\varnothing$ and $e\left(Q_{1}\right) \geq \delta / 2^{j}$. Now we define subcollections $\widetilde{\mathscr{P}}_{j} \subset \mathscr{P}_{j}$ as follows: $\widetilde{\mathscr{P}_{0}}=\varnothing$, and, assuming $\widetilde{\mathscr{P}}_{0}, \cdots, \widetilde{\mathscr{P}}_{j-1}$ are already defined, let $\widetilde{\mathscr{P}}_{j}$ be the collection of all $P \in \mathscr{P}_{j}$ which are not contained in $\bigcup_{i<j}\left(\bigcup_{P \in \widetilde{\mathscr{P}}_{i}} P\right)$. Let $\widetilde{\mathscr{P}}=\bigcup_{j=1}^{\infty} \widetilde{\mathscr{P}}_{j}$. Notice that $\bigcup_{P \in \widetilde{\mathscr{P}}} P=\bigcup_{P \in \mathscr{P}}$ and that the cubes in $\widetilde{\mathscr{P}}$ have pairwise disjoint interiors. We divide each $\widetilde{\mathscr{P}}_{j}$ into three subcollections:

$\mathscr{G}_{j}=$ those cubes $P \in \widetilde{\mathscr{P}}_{j}$ which contain a cube $Q_{1}$, where (as in (1)) $Q_{1}$ is contained in some $Q \in \mathscr{Q}_{j}$ and $F \cap Q_{1}=\varnothing, e\left(Q_{1}\right) \geq \delta / 2^{j}$, $\mathscr{B}_{j}=$ those cubes $P \in \widetilde{\mathscr{P}}_{j} \backslash \mathscr{G}_{j}$ which are such that there is $P_{1} \in \widetilde{\mathscr{P}}_{j}$ as in (1), and

$$
\mathscr{V}_{j}=\widetilde{\mathscr{P}}_{j} \backslash\left(\mathscr{G}_{j} \cup \mathscr{B}_{j}\right) \text {. }
$$

Let $\mathscr{G}=\bigcup_{j=1}^{\infty} \mathscr{G}_{j}, \mathscr{B}=\bigcup_{j=1}^{\infty} \mathscr{B}_{j}$, and $\mathscr{V}=\bigcup_{j=1}^{\infty} \mathscr{V}_{j}$. We claim that

$$
\bigcup_{P \in \mathscr{V}} P \subset \bigcup_{P \in \mathscr{B} \cup \mathscr{G}} P^{(3)} \quad \text { and } \quad \bigcup_{P \in \mathscr{B}} P \subset \bigcup_{P \in \mathscr{G}} P^{(3)} .
$$

In fact the second inclusion is true by the definitions of $\mathscr{B}$ and $\mathscr{G}$. To prove the first inclusion, take a cube $P_{j} \in \mathscr{V}_{j}$. Since $\mathscr{V}_{i_{0}}=\varnothing$ for the smallest $i_{0}$ such that $\widetilde{\mathscr{P}}_{i_{0}} \neq \varnothing$ and any cube in a $\mathscr{V}_{q}$ must intersect a cube in $\widetilde{\mathscr{P}}_{q^{\prime}}$ for some $q^{\prime}<q$ by virtue of property (1), by definition we can find a sequence $\left\{P_{j_{q}} \in \widetilde{\mathscr{P}}_{j_{q}}\right\}_{q=1, \cdots, N}$ with $j=j_{1}>\cdots>j_{N}=j-i$, $i \geq N-1 \geq 1, P_{j-i} \in \mathscr{B}_{j-i} \cup \mathscr{G}_{j-i}, P_{j_{q}} \cap P_{j_{q+1}} \neq \varnothing$ and with $P_{j_{q}} \in \mathscr{V}_{j_{q}}$ for all $q=1, \cdots, N-1$. Notice that then the sum of the edge lengths $\sum_{q=1}^{N-1} e\left(P_{j_{q}}\right)$ is $\leq \frac{1}{2} \sum_{q=0}^{i-1} 2^{-q} e\left(P_{j-i}\right)<e\left(P_{j-i}\right)$, so that $P_{j} \subset P_{j-i}^{(3)}$, thus establishing the first inclusion of (2).

Now by (2) we have

$$
\begin{aligned}
\sum_{P \in \widetilde{\mathscr{P}}} e(P)^{m} & =\sum_{P \in \mathscr{G}} e(P)^{m}+\sum_{P \in \mathscr{B}} e(P)^{m}+\sum_{P \in \mathscr{V}} e(P)^{m} \\
& \leq\left(1+3^{m}+2.3^{2 m}\right) \sum_{P \in \mathscr{G}} e(P)^{m} \leq 3^{2 m+1} \sum_{P \in \mathscr{G}} e(P)^{m}
\end{aligned}
$$


On the other hand since each $P \in \mathscr{G}_{j}$ contains a cube $Q_{P}$ of edge length $\delta / 2^{j} \equiv 2^{-(j+N)}$ with $Q_{P} \cap F=\varnothing$, by subdividing each of the cubes in $P \in \widetilde{\mathscr{P}}_{j}$ into $2^{-(N+1) m}$ congruent subcubes (each of edge length $2^{-N-j-1}$ ), we obtain a new collection $\widehat{\mathscr{P}}$ with

$$
\sum_{P \in \widehat{\mathscr{P}}} e(P)^{m}=\sum_{P \in \widetilde{\mathscr{P}}} e(P)^{m} \leq 3^{2 m+1} \sum_{P \in \mathscr{G}} e(P)^{m}
$$

and

$$
\sum_{P \in \widehat{\mathscr{P}}, P \cap F=\varnothing} e(P)^{m} \geq 2^{-(N+1) m} \sum_{P \in \mathscr{G}} e(P)^{m} \equiv\left(\frac{\delta}{2}\right)^{m} \sum_{P \in \mathscr{G}} e(P)^{m} .
$$

Thus if we let

$$
\widetilde{\mathscr{Q}}=\{P \in \widehat{\mathscr{P}}: P \cap F \neq \varnothing\},
$$

then

$$
\sum_{Q \in \widetilde{Q}} e(Q)^{m} \leq 3^{2 m+1} \sum_{P \in \mathscr{G}} e(P)^{m}
$$

and

$$
\sum_{P \in \widehat{\mathscr{P}} \mid \widetilde{\mathscr{Q}}} e(P)^{m} \geq\left(\frac{\delta}{2}\right)^{m} \sum_{P \in \mathscr{G}} e(P)^{m} \geq \delta^{2 m-1} \sum_{Q \in \widetilde{\mathscr{Q}}} e(Q)^{m}, \quad \text { by (3). }
$$

Since $\sum_{P \in \widehat{\mathscr{P}}} e(P)^{m} \leq 1,(4)$ gives

$$
\sum_{Q \in \widetilde{Q}} e(Q)^{m} \leq\left(1+\delta^{2 m-1}\right)^{-1} \leq 1-\delta^{2 m} .
$$

Furthermore by construction each $\widetilde{Q} \in \widetilde{\mathscr{Q}}$ is contained in $Q^{(5)}$ for some $Q \in \mathscr{Q}$ with $2^{-(N+2)} e(Q) \leq e(\widetilde{Q})<e(Q)$, so the proof is complete.

\section{3. $L^{2}$-estimates}

The main $L^{2}$-estimates are as follows.

3.1. Theorem (Main $L^{2}$ estimates). Suppose $\gamma, \tau, \alpha \in(0,1)$ are given. There are $\varepsilon_{0}=\varepsilon_{0}\left(\mathbf{C}^{(0)}, \mathscr{M}, \gamma, \tau\right), \beta_{0}=\beta_{0}\left(\mathbf{C}^{(0)}\right) \in(0,1)$ such that if $\varepsilon \leq \varepsilon_{0}, \mathbf{C}=\mathbf{C}_{0} \times \mathbf{R}^{m} \in \mathscr{C}_{\varepsilon}\left(\mathbf{C}^{(0)}\right), M \in \mathscr{N}_{\varepsilon}\left(\mathbf{C}^{(0)}\right)$, and $U, u$ are as in Lemma 2.6 with $\beta=\beta_{0}$, then the following inequalities hold for all $Z=(\xi, \eta) \in B_{3 / 4}$ with $\boldsymbol{\Theta}_{M}(Z) \geq \boldsymbol{\Theta}_{\mathbf{C}^{(0)}}(0)$ : 
(i)

$$
\begin{aligned}
& |\xi|^{2}+\int_{M \cap B_{y}} \sum_{j=1}^{m}\left|e_{l+k+j}^{\perp}\right|^{2}+\int_{M \cap B_{y}} \frac{d^{2}}{|X-Z|^{n-\alpha}} \\
& \quad+\int_{U \cap B_{\gamma}} R^{2-n}\left(\frac{\partial(u / R)}{\partial R}\right)^{2} \leq C \int_{M \cap B_{1}} d^{2}, R=R(x, y)=\sqrt{|x|^{2}+|y|^{2}}, \\
& \text { (ii) } \int_{\left\{X=(x, y) \in \mathbf{C} \cap B_{y}:|x|>\tau\right\}} \frac{\left|u(x, y)-\xi^{\perp}(x, y)\right|^{2}}{|X-Z|^{n+2-\alpha}} \leq C \int_{M \cap B_{1}} d^{2},
\end{aligned}
$$

where $\xi^{\perp}(x, y)$ means orthogonal projection of $(\xi, 0)$ onto $\left(T_{X} \mathbf{C}\right)^{\perp}$, and $C=C\left(\gamma, \alpha, \mathbf{C}^{(0)}, \mathscr{M}\right)>0$ is a positive constant not depending on $\tau$; notice that $\xi^{\perp}(x, y) \equiv \sum_{j=1}^{l+k} \xi^{j} e_{j}^{\perp}(x, y)$ depends only on $\omega=|x|^{-1} x$ and not on $|x|$ or $y$.

Before we prove the theorem we state and prove an important corollary.

3.2. Corollary. Let $\alpha, \tau, \delta \in\left(0, \frac{1}{4}\right)$ be given. There are $\varepsilon_{0}=$ $\varepsilon_{0}\left(\mathbf{C}^{(0)}, \mathscr{M}, \tau\right), \beta_{0}=\beta_{0}\left(\mathbf{C}^{(0)}\right) \in(0,1)$ such that if $\mathbf{C} \in \mathscr{C}_{\varepsilon}\left(\mathbf{C}^{(0)}\right), M \in$ $\mathscr{N}_{\varepsilon}\left(\mathbf{C}^{(\theta)}\right)$ with $\varepsilon \leq \min \left\{\varepsilon_{0}, \delta\right\}, u$ is as in Lemma 2.6 with $\beta=\beta_{0}$ and $\gamma=\frac{3}{4}$, and hypothesis $(* *)$ of Remark 1.14 holds with $\delta_{0}=\delta$, then

$$
\begin{gathered}
\int_{\left\{(x, y)=(r \omega, y) \in \mathbf{C} \cap B_{1 / 2}: r>\tau\right\}} \frac{|u-\kappa|^{2}}{r_{\delta}^{l+2-\alpha}} \leq C \int_{M \cap B_{1}} d^{2}, \\
\int_{M \cap B_{1 / 2}} \frac{d^{2}}{r_{\delta}^{l-\alpha}} \leq C \int_{M \cap B_{1}} d^{2},
\end{gathered}
$$

where $C=C\left(\alpha, \mathscr{M}, \mathbf{C}^{(0)}\right), r_{\delta}=\max \{r, \delta\} \quad(\equiv \max \{|x|, \delta\}), \kappa(x, y)=$ $\sum_{j=1}^{l+k} \kappa_{j}(r, y) e_{j}^{\perp}$ with $\kappa_{j}:(0,1) \times B_{1}^{m}(0) \rightarrow \mathbf{R}$ satisfying $\sup \left|\kappa_{j}\right|^{2} \leq$ $C \int_{M \cap B_{1}} d^{2}$, and $e_{j}^{\perp}(x, y)$ is the orthogonal projection of $e_{j}$ onto $\left(T_{(x, y)} \mathbf{C}\right)^{\perp}$.

3.3. Remark. Notice that (ii) implies

$$
\int_{M \cap\left(B_{\delta}^{l+k} \times \mathbf{R}^{m}\right) \cap B_{1 / 2}} d^{2} \leq C \delta^{l-\alpha} \int_{M \cap B_{1}} d^{2}
$$

with $C$ independent of $\delta$, so that the part of $M \cap B_{1 / 2}$ close to the subspace $\{0\} \times \mathbf{R}^{m}$ contributes little to $\int_{M \cap B_{1}} d^{2}$ if the hypotheses of Corollary 3.2 hold with $\delta$ small enough (depending only on $\mathbf{C}^{(0)}, \mathscr{M}$ ).

Proof of 3.2. To prove (ii), note that (i) of Theorem 3.1 implies that

$$
\rho^{-n+\alpha} \int_{M \cap B_{\rho}(0, z)} d^{2} \leq C \int_{M \cap B_{1}} d^{2}, \quad \rho \in\left(\delta, \frac{1}{4}\right),
$$


for each $z \in B_{1 / 2}^{m}$, because by $(* *)$ of Remark 1.14 for any such $z$ we have $Z \in\left\{X: \boldsymbol{\Theta}_{M}(X) \geq \boldsymbol{\Theta}_{\mathbf{C}^{(0)}}(0)\right\}$ with $|Z-(0, z)|<\delta$. Since we can cover $B_{1 / 2} \cap B_{\rho / 2}^{l+k} \times \mathbf{R}^{m}$ by $N \leq C(n, k) \rho^{-m}$ balls $B_{\rho}\left(0, z_{j}\right)$ with $\left|z_{j}\right| \leq \frac{1}{2}$ such that the collection $\left\{B_{\rho}\left(0, z_{j}\right)\right\}$ decomposes into $\leq C(n, k)$ pairwise disjoint subcollections, this implies that

$$
\rho^{-l+\alpha} \int_{M \cap B_{1 / 2} \cap\left(B_{\rho / 2}^{l+k} \times \mathbf{R}^{m}\right)} d^{2} \leq C \int_{M \cap B_{1}} d^{2}, \quad \rho \in\left(\delta, \frac{1}{4}\right),
$$

and the required result (with $2 \alpha$ in place of $\alpha$ ) follows by multiplying by $\rho^{-1+\alpha}$ and integrating with respect to $\rho$.

To prove (i), for $\rho \in\left(\delta, \frac{1}{4}\right)$ let $B_{\rho}\left(0, z_{j}\right)$ be the same cover as we used above in the proof of (ii). By inequality (ii) of Theorem 3.1 for each $j$ we have $\xi_{j}=\left(\xi_{j}^{1}, \cdots, \xi_{j}^{m}\right) \in \mathbf{R}^{m}$ such that

$$
\rho^{-n-2+\alpha} \int_{U_{\tau} \cap B_{\rho}\left(0, z_{j}\right)}\left|u(r \omega, y)-\sum_{i=1}^{l+k} \xi_{j}^{i} e_{i}^{\perp}\right|^{2} \leq C \int_{M \cap B_{1}} d^{2},
$$

where $\sum_{i=1}^{l+k}\left(\xi_{j}^{i}\right)^{2} \leq C \int_{M \cap B_{1}} d^{2}$ and $U_{\tau}=\left\{(x, y) \in \mathbf{C} \cap B_{1 / 2}:|x|>\right.$ $\tau\}$. Thus if for each fixed $(r, y)$ we let $\kappa^{i}(r, y)$ be selected so that $\sum_{i=1}^{l+k}\left(\kappa^{i}(r, y)\right)^{2} \leq C \int_{M \cap B_{1}} d^{2}$ and

$$
\int_{\Sigma}\left|u(r \omega, y+)-\sum_{i=1}^{l+k} \kappa^{i}(r, y) e_{i}^{\perp}\right|^{2} d \omega=\inf \int_{\Sigma}\left|u(r \omega, y)-\sum_{i=1}^{l+k} \lambda^{i} e_{i}^{\perp}\right|^{2} d \omega,
$$

where the inf is over all $\lambda \in \mathbf{R}^{l+k}$ with $\sum_{i=1}^{l+k}\left(\lambda^{i}\right)^{2} \leq C \int_{M \cap B_{1}} d^{2}$, and $\Sigma=\mathbf{C}_{0} \cap S^{l+k-1}$, then from (3) it follows that

(4) $\rho^{-n-2+\alpha} \int_{U_{\tau} \cap B_{\rho}\left(0, z_{j}\right) .}\left|u(x, y)-\sum_{i=1}^{l+k} \kappa^{i}(r, y) e_{i}^{\perp}\right|^{2} \leq C \int_{M \cap B_{1}} d^{2} \forall j$.

Summing over $j$ in (4) we thus get

$$
\rho^{-l-2+\alpha} \int_{U_{\tau} \cap\left(B_{\rho / 2}^{l+k} \times \mathbf{R}^{m}\right)}\left|u(x, y)-\sum_{i=1}^{l+k} \kappa^{i}(r, y) e_{i}^{\perp}\right|^{2} \leq C \int_{M \cap B_{1}} d^{2},
$$

and again the required inequality is obtained by multiplication by $\rho^{-1+\alpha}$ and integrating with respect to $\rho$.

Theorem 3.1 will be proved by combining the two Lemmas 3.4 and 3.9 below.

3.4. Lemma. Suppose $\alpha, \gamma, \tau \in(0,1)$. There are $\varepsilon_{0}=\varepsilon_{0}\left(\mathbf{C}^{(0)}, \mathscr{M}, \tau, \gamma\right)$ and $\beta_{0}=\beta_{0}\left(\mathbf{C}^{(0)}\right)>0$ such that if $\mathbf{C}=\mathbf{C} \times \mathbf{R}^{m} \in \mathscr{C}_{\varepsilon}\left(\mathbf{C}^{(0)}\right), M \in \mathscr{N}_{\varepsilon}\left(\mathbf{C}^{(0)}\right)$ 
with $\varepsilon \leq \varepsilon_{0}, \Theta_{M}(0) \geq \Theta_{\mathbf{C}^{(0)}}(0)$, and $U, u$ are as in Lemma 2.6 with $\beta=\beta_{0}$, then

$$
\begin{aligned}
\int_{U \cap B_{\gamma}} R^{2-n}\left(\frac{\partial(u / R)}{\partial R}\right)^{2}+\int_{M \cap B_{\gamma}} R^{-n-2}\left|(x, y)^{\perp}\right|^{2} \\
\quad+\int_{M \cap B_{\gamma}} \sum_{j=1}^{m}\left|e_{l+k+j}^{\perp}\right|^{2}+\int_{M \cap B_{\gamma}} R^{-n-2+\alpha} d^{2} \\
\leq C \int_{M \cap B_{1}} d^{2}
\end{aligned}
$$

where $C=C\left(\mathbf{C}^{(0)}, \alpha, \gamma\right), R=R(x, y)=\sqrt{|x|^{2}+|y|^{2}},(x, y)^{\perp}$ means orthogonal projection of the radial vector $(x, y)$ onto the normal space $\left(T_{(x, y)} M\right)^{\perp}$ of $M$ at $(x, y)$, and $d(X)=\operatorname{dist}(X, \mathbf{C})$.

Proof. Recall (see, e.g., [1] or [19]) the following version of the monotonicity identity for $M \in \mathscr{M}$; namely, if $M \in \mathscr{M}$ with $U_{M} \supset B_{\rho}$, then

(1) $n \rho^{n-1} \int_{M \cap B_{\rho}} R^{-n-2}\left|(x, y)^{\perp}\right|^{2}=\frac{d}{d \rho} \int_{M \cap B_{\rho}}\left|\nabla^{M} R\right|^{2}-n \Theta_{M}(0) \rho^{n-1}$

$$
\leq \frac{d}{d \rho}\left(\mathscr{H}^{n}\left(M \cap B_{\rho}\right)-\left|\mathbf{C}^{(0)} \cap B_{\rho}\right|\right), \quad \text { a.e. } \rho \in(0,1] \text {. }
$$

If $\psi: \mathbf{R} \rightarrow[0,1]$ is a decreasing $C^{1}$ function with $\psi \equiv 1$ on $(-\infty$, $(1+\gamma) / 2)$ and $\psi \equiv 0$ on $((3+\gamma) / 4, \infty)$, by multiplication by $\psi^{2}(\rho)$ and integration with respect to $\rho$ this implies

$$
\int_{0}^{1} \psi^{2}(\tau) \tau^{n-1} \int_{M \cap B_{\tau}} R^{-n-2}\left|(x, y)^{\perp}\right|^{2} d \tau \leq \int_{M \cap B_{1}} \psi^{2}(R)-\int_{\mathbf{C}^{(0)} \cap B_{1}} \psi^{2}(R)
$$

Since $\psi \equiv 1$ on $[\gamma,(1+\gamma) / 2]$, this gives

(2) $\gamma^{n-1} \frac{1-\gamma}{2} \int_{M \cap B_{\gamma}} R^{-n-2}\left|(x, y)^{\perp}\right|^{2} \leq \int_{M \cap B_{1}} \psi^{2}(R)-\int_{\mathbf{C}^{(0)} \cap B_{1}} \psi^{2}(R)$.

Now we use (2.5) with $\psi=\psi(R)$, so that $D_{x^{i}} \psi=x^{i} \psi^{\prime} / R$ and $D_{y^{i}} \psi=$ $y^{i} \psi^{\prime} / R$. Then (2.5) yields 
(3)

$$
\begin{aligned}
\int_{M \cap B_{1}} & \left(l+\frac{1}{2} \sum_{j=1}^{m}\left|e_{l+k+j}^{\perp}\right|^{2}\right) \psi^{2}(R) \\
\leq & C \int_{M \cap B_{1}}\left|(x, 0)^{\perp}\right|^{2}\left(\psi^{\prime}\right)^{2}-2 \int_{M \cap B_{1}} R^{-1} \psi(R) \psi^{\prime}(R) \sum_{i, j=1}^{l+k} g^{i j} x^{i} x^{j} \\
\leq & C \int_{M \cap B_{1}}\left|(x, 0)^{\perp}\right|^{2}\left(\psi^{2}(R)+\left(\psi^{\prime}(R)\right)^{2}\right)-2 \int_{M \cap B_{1}} r^{2} R^{-1} \psi(R) \psi^{\prime}(R),
\end{aligned}
$$

where $(x, 0)^{\perp}$ means orthogonal projection of $(x, 0)$ onto $\left(T_{(x, y)} M\right)^{\perp}$ for any point $(x, y) \in M$, and we use the fact that

$$
\sum_{i, j=1}^{l+k} g^{i j} x^{i} x^{j}=r^{2}+\sum_{i, j=1}^{l+k}\left(g^{i j}-\delta_{i j}\right) x^{i} x^{j}=r^{2}-\left|(x, 0)^{\perp}\right|^{2} .
$$

If $(x, y)=\left(x^{\prime}, y\right)+u\left(x^{\prime}, y\right) \in G \equiv \operatorname{graph} u$, then

$$
(x, 0)^{\perp}=u\left(x^{\prime}, y\right)+\left(P_{(x, y)}-Q_{\left(x^{\prime}, y\right)}\right)(x, 0),
$$

where $P_{(x, y)}$ and $Q_{\left(x^{\prime}, y\right)}$ denote respectively the orthogonal projection onto $\left(T_{(x, y)} M\right)^{\perp}$ and $\left(T_{\left(x^{\prime}, y\right)} C\right)^{\perp}$. Since $|\nabla u| \leq 1$, we have $\| P_{(x, y)}$ $Q_{\left(x^{\prime}, y\right)} \| \leq C\left|\nabla u\left(x^{\prime}, y\right)\right|, C=C(n, k)$, and hence from (3) and (4) it follows that

$$
\begin{aligned}
\int_{M \cap B_{1}} & \left(l+\frac{1}{2} \sum_{j=1}^{m}\left|e_{l+k+j}^{\perp}\right|^{2}\right) \psi^{2}(R) \leq C \int_{U \cap B_{\hat{\gamma}}}\left(|u|^{2}+r^{2}|\nabla u|^{2}\right) \\
+ & C \int_{M \cap B_{\hat{\gamma}} \backslash G} r^{2}-2 \int_{G \cap B_{1}} r^{2} R^{-1} \psi(R) \psi^{\prime}(R),
\end{aligned}
$$

where $G=\operatorname{graph} u$ and $\tilde{\gamma}=(3+\gamma) / 4$. Now since $\mathbf{C}_{0}$ is a smooth cone with volume element $r^{l-1} d r d \omega$ where $d \omega$ denotes the volume element of $\Sigma$, by a one-dimensional integration by parts we deduce that

$$
l \int_{\mathbf{C}_{0}} \varphi^{2}(r)=-2 \int_{\mathbf{C}_{0}} r \varphi(r) \varphi^{\prime}(r),
$$

provided $\varphi \in C^{\infty}(0, \infty)$ with $\varphi \equiv$ constant in a neighborhood of 0 and $\varphi \equiv 0$ in a neighborhood of $\infty$. Using this with $\varphi(r)=\psi(R), R=$ $\sqrt{r^{2}+|y|^{2}}$ for each fixed $y$ and then noting that $\varphi^{\prime}(r)=r^{-1} \psi^{\prime}(R)$, after 
an integration with respect to $y$ we conclude that

$$
l \int_{\mathbf{C} \cap B_{1}} \psi^{2}(R)=-2 \int_{\mathbf{C} \cap B_{1}} r^{2} R^{-1} \psi(R) \psi^{\prime}(R) .
$$

We also need to note that the volume element of $G$ is $\sqrt{g} r^{l-1} d r d \omega$, where $\sqrt{g}=1+E$, with $|E| \leq C\left(r^{-2}|u|^{2}+|\nabla u|^{2}\right)$, and that $r, R$ at a point $(x, y)+u(x, y) \in G$ are given by $r^{2}=|x|^{2}+|u(x, y)|^{2}$ and $R^{2}=|x|^{2}+|u(x, y)|^{2}+|y|^{2}$ respectively. Thus for example

$$
\int_{G \cap B_{1}} r^{2} R^{-1} \psi(R) \psi^{\prime}(R)=\int_{U \cap B_{1}} r_{u}^{2} R_{u}^{-1} \psi\left(R_{u}\right) \psi^{\prime}\left(R_{u}\right) \sqrt{g},
$$

where $r_{u}^{2}=|x|^{2}+|u(x, y)|^{2}$ and $R_{u}^{2}(x, y)=|x|^{2}+|u(x, y)|^{2}+|y|^{2}$, and by using (6) in (5), and keeping in mind that $|u(x, y)| \leq|x|$ in $U$ by definition, we deduce that

$$
\begin{aligned}
(2 l)^{-1} & \int_{M \cap B_{\gamma}} \sum_{j=1}^{m}\left|e_{l+k+j}^{\perp}\right|^{2}+\int_{M \cap B_{1}} \psi^{2}(R)-\int_{\mathbf{C} \cap B_{1}} \psi^{2}(R) \\
& \leq C \int_{U \cap B_{\tilde{\gamma}}}\left(|u|^{2}+r^{2}|\nabla u|^{2}\right)+C \int_{M \cap B_{\tilde{\gamma}} \backslash G} r^{2},
\end{aligned}
$$

and by (2) and Lemma 2.6 with $\tilde{\gamma}$ in place of $\gamma$ we obtain

$$
\int_{M \cap B_{\gamma}} R^{-n-2}\left|(x, y)^{\perp}\right|^{2}+\int_{M \cap B_{\gamma}} \sum_{j=1}^{m}\left|e_{l+k+j}^{\perp}\right|^{2} \leq C \int_{M \cap B_{1}} d^{2} .
$$

Next we establish the required bound for $\int_{M \cap B_{\gamma}} R^{-n-2+\alpha} d^{2}$. First notice that $d: \mathbf{R}^{n+k} \rightarrow \mathbf{R}$, defined by $d(X)=\operatorname{dist}(X, \mathbf{C})$, is a homogeneous degree 1 function of $|X|$ with Lipschitz constant $\leq 1$, as a function of $X=(x, y)$ is independent of the $y$-variable, and is smooth in $x$ in the conical region

$$
K_{\varepsilon_{0}} \equiv\left\{(x, y) \in \mathbf{R}^{n+k} \backslash\{0\} \times \mathbf{R}^{m}: \operatorname{dist}(X, \mathbf{C}) \leq \varepsilon_{0}|x|\right\}
$$

provided $\varepsilon_{0}$ is small enough, depending only on $\mathbf{C}^{(0)}$. Thus we can easily construct a smooth homogeneous degree 1 function $\tilde{d}: \mathbf{R}^{n+k} \rightarrow \mathbf{R}$ with $\tilde{d} \equiv d$ in the conical region $K_{\varepsilon_{0}}$ and with $C^{-1} d(X) \leq \tilde{d}(X) \leq C d(X)$ and $\operatorname{Lip} \tilde{d} \leq C$ everywhere, where $C=C\left(\mathbf{C}^{(0)}\right)$.

Now in the identity (1.1) take $\Phi(X)=\zeta^{2} R^{-n+\alpha} \tilde{d}^{2} X / R^{2}$, where $\zeta \in$ $C^{\infty}\left(\mathbf{R}^{n+k}\right)$ with $\zeta \equiv 1$ on $B_{(1+\gamma) / 2}, \zeta \equiv 0$ outside $B_{1}$, and $\left|\nabla^{\mathbf{R}^{n+k}} \zeta\right| \leq C$, 
$C=C(\gamma)$. Notice that $\tilde{d}^{2} / R^{2}$ is smooth and homogeneous degree zero away from the singular axis $\{0\} \times \mathbf{R}^{m}$, and hence

$$
\sum_{j=1}^{n+k} X^{j} D_{j} \frac{\tilde{d}^{2}}{R^{2}}=0
$$

Of course since $\operatorname{Lip} \tilde{d} \leq C$ and $\tilde{d} \leq C R$ we also have

$$
\left|\nabla^{\mathbf{R}^{n+k}} \tilde{d} / R\right| \leq 2 C / R .
$$

Since $\operatorname{div}_{M} X \equiv n$ and $\left|\nabla^{M} R\right| \leq\left|\nabla^{\mathbf{R}^{n+k}} R\right| \leq 1$, (1.1) in combination with (8) implies

$$
\begin{aligned}
& \alpha \int_{M \cap B_{1}} \zeta^{2} R^{-n-2+\alpha} \tilde{d}^{2} \\
& \quad \leq \int_{M \cap B_{1}} R^{-n+\alpha} \sum_{i, j=1}^{n+k} X^{i}\left(\left(\delta_{i j}-g^{i j}\right) 2 \zeta^{2} \frac{\tilde{d}}{R} D_{j} \frac{\tilde{d}}{R}-2 g^{i j} \zeta \frac{\tilde{d}^{2}}{R^{2}} D_{j} \zeta\right),
\end{aligned}
$$

and by Cauchy's inequality and (9) this yields

$$
\int_{M \cap B_{y}} R^{-n-2+\alpha} d^{2} \leq C \int_{M \cap B_{1}}\left(\zeta^{2} R^{-n-2+\alpha}\left|(x, y)^{\perp}\right|^{2}+R^{-n+\alpha} d^{2}\left|\nabla^{M} \zeta\right|^{2}\right),
$$

with $C=C\left(\mathbf{C}^{(0)}, \alpha\right)$. Using (7) with $(1+\gamma) / 2$ in place of $\gamma$, we then have the required inequality.

It remains only to prove the bound

$$
\int_{U \cap B_{y}} R^{2-n}\left(\frac{\partial u / R}{\partial R}\right)^{2} \leq C \int_{M \cap B_{1}} d^{2} .
$$

This is proved as follows. First we note that the expression $(x, y)^{\perp}$ appearing in the inequality (7) can be written on graph $u$ as $\left(x^{\prime}+u\left(x^{\prime}, y\right), y\right)^{\perp}$, where $x^{\prime}$ denotes the nearest point projection of $x$ onto $\mathbf{C}_{0}$. Now with $R=\left|\left(x^{\prime}, y\right)\right|$ and $\Phi(x, y)=(x, y) \equiv\left(x^{\prime}+u\left(x^{\prime}, y\right), y\right)$ we see that, because $\Phi$ maps $C$ into $M$, we must have $\partial \Phi\left(x^{\prime}, y\right) / \partial R \in T_{(x, y)} M$, and hence $\left(\partial\left(\Phi\left(x^{\prime}, y\right) / R\right) / \partial R\right)^{\perp}$ is the same as $-R^{-2}(x, y)^{\perp}$. But on the other hand, since $R^{-1}\left(x^{\prime}, y\right)$ is homogeneous of degree zero with respect to $R$, the expression $\partial\left(\Phi\left(x^{\prime}, y\right) / R\right) \partial R$ is just $\partial\left(u\left(x^{\prime}, y\right) / R\right) / \partial R$, and hence we obtain the identity

$$
(x, y)^{\perp}=-R^{2}\left(\frac{\partial}{\partial R}\left(\frac{u(x, y)}{R}\right)\right)^{\perp}, \quad\left(x^{\prime}, y\right) \in U .
$$


However, $u\left(x^{\prime}, y\right)$ is normal to $\mathrm{C}$ at $\left(x^{\prime}, y\right)$, and $\left\|P_{(x, y)}-Q_{\left(x^{\prime}, y\right)}\right\| \leq \frac{1}{2}$ provided $\beta_{0}=\beta_{0}\left(\mathbf{C}^{(0)}\right)$ is sufficiently small where $P_{(x, y)}, Q_{\left(x^{\prime}, y\right)}$ are as in (4). Thus

$$
\left|(x, y)^{\perp}\right| \geq \frac{1}{2} R^{2}\left|\frac{\partial}{\partial R}\left(\frac{u\left(x^{\prime}, y\right)}{R}\right)\right|,
$$

and the remaining inequality (10) follows from (7) and (11).

In the following lemma we are going to consider a point $Z+(\xi, \eta) \in$ $\operatorname{sing} M \cap B_{3 / 4}$, where $M \in \mathscr{N}_{\varepsilon}\left(\mathbf{C}^{(0)}\right)$. Notice that then by Lemma 2.6 we have $|\xi|=\operatorname{dist}\left(Z,\{0\} \times \mathbf{R}^{m}\right) \leq \delta$, with $\delta=\delta\left(\varepsilon, \mathbf{C}^{(0)}\right) \rightarrow 0$ as $\varepsilon \downarrow 0$. If we take suitable $\varepsilon_{0}=\varepsilon_{0}\left(\mathbf{C}^{(0)}\right), \theta=\theta\left(\mathbf{C}^{(0)}\right) \in(0,1)$, and if $\mathbf{C}=\mathbf{C}_{0} \times \mathbf{R}^{m} \in$ $\mathscr{E}_{\varepsilon_{0}}\left(\mathbf{C}^{(0)}\right)$ and $X=(x, y) \in \mathbf{R}^{n+k}$ with $|x| \geq \theta^{-1}(|\xi|+d(X))$, then

$$
\operatorname{dist}\left(X, \tau_{z} \mathbf{C}\right)=\left|(x, y)-\left(x^{\prime}, y\right)-\xi^{\perp}\right|+R,
$$

where $x^{\prime}$ is the nearest point projection of $x$ onto $\mathbf{C}_{0}, \tau_{Z}$ the translation $X \mapsto X-Z, \xi^{\perp}$ is the orthogonal projection of $(\xi, 0)$ onto $\left(T_{\left(x^{\prime}, 0\right)} \mathbf{C}\right)^{\perp}$, and

$$
|R| \leq C|x|^{-1}|\xi|^{2}, \quad C=C\left(\mathbf{C}^{(0)}\right) .
$$

Since $\left|(x, y)-\left(x^{\prime}, y\right)\right|=\operatorname{dist}(X, \mathbf{C})$,

$$
\left|\operatorname{dist}\left(X, \tau_{Z} \mathbf{C}\right)\right| \geq\left|\xi^{\perp}\right|-\operatorname{dist}(X, \mathbf{C})-|R| .
$$

Notice also that by the triangle inequality,

$$
\left|\operatorname{dist}\left(X, \tau_{Z} \mathbf{C}\right)-\operatorname{dist}(X, \mathbf{C})\right| \leq|\xi|
$$

for all $X \in \mathbf{R}^{n+k}$, because $\tau_{Z} \mathbf{C} \equiv \tau_{(\xi, 0)} \mathbf{C}$.

3.9. Lemma. There is $\varepsilon_{0}=\varepsilon_{0}\left(\mathbf{C}^{(0)}, \mathscr{M}\right)>0$ such that if $\mathbf{C}=\mathbf{C}_{0} \times \mathbf{R}^{m} \in$ $\mathscr{C}_{\varepsilon}\left(\mathbf{C}^{(0)}\right)$ and $M \in \mathscr{N}_{\varepsilon}\left(\mathbf{C}^{(0)}\right)$ with $\varepsilon \leq \varepsilon_{0}$, then for any $Z \in \operatorname{sing} M \cap B_{1 / 2}$ with $\Theta_{M}(Z) \geq \Theta_{C^{(0)}}(0)$ we have the inequality

$$
\operatorname{dist}^{2}\left(Z,\{0\} \times \mathbf{R}^{m}\right)+\int_{M \cap B_{1}} d_{Z}^{2} \leq C \int_{M \cap B_{1}} d^{2}, \quad C=C\left(\mathbf{C}^{(0)}, \mathscr{M}\right),
$$

where $d_{Z}(X) \equiv \operatorname{dist}\left(X, \tau_{Z} \mathbf{C}\right)$, with $\tau_{Z}$ the translation $X \mapsto X-Z$.

Proof of 3.9. Since $M \in \mathscr{N}_{\varepsilon_{0}}\left(\mathbf{C}^{(0)}\right)$ and the cross section $\mathbf{C}_{0}^{(0)}$ has only the isolated singularity at 0 , we must have a constant $\delta_{1}\left(\mathbf{C}^{(0)}, \mathscr{M}\right)$ such 
that for every $a \in \mathbf{R}^{l+k}, \rho \in\left(0, \frac{1}{4}\right)$ and $Z \in \operatorname{sing} M \cap B_{1 / 2}$, there exists $\varepsilon_{0}=\varepsilon_{0}\left(\rho, \mathbf{C}^{(0)}\right)$ so that

$$
\left|a^{\perp}\right| \geq \delta_{1}|a| \text { on a set of measure } \geq \delta_{1} \rho^{n} \text { in } M \cap B_{\rho}(Z) ;
$$

here, as in (3.5), $a^{\perp}(X)$ is the orthogonal projection of $a$ onto $\left(T_{x^{\prime}} \mathbf{C}_{0}\right)^{\perp}$ for $X=(x, y) \in M \cap B_{1}$ with $|x| \geq C d(X)$. Indeed if no such $\delta_{1}$ exists we would have (1) failing with $\delta=j^{-1}$ and with sequences $a_{j} \in$ $S^{l+k-1}, \varepsilon_{j} \downarrow 0, M_{j}, \mathbf{C}_{j} \in \mathscr{C}_{\varepsilon_{j}}\left(\mathbf{C}^{(0)}\right), Z_{j}$ in place of $a, \varepsilon_{0}, M, \mathbf{C}$ and $Z$ respectively. Thus $\left|a_{j}^{\perp}\right|<j^{-1}\left|a_{j}\right|$ except on a set of measure $<j^{-1} \rho^{n}$ in $M_{j} \cap B_{\rho}\left(Z_{j}\right)$. After passing to a subsequence we have $a_{j} \rightarrow a \in S^{l+k-1}$ which together with Lemma 2.6 shows that $Z_{j} \rightarrow(0, z)$ for some $z$ with $|z| \leq \frac{1}{2}$. Then from 1.3(b) and Lemma 2.6 it follows that $a^{\perp} \equiv 0$ on $\mathbf{C}_{0}^{(0)}$, where $a^{\perp}(x, y)$ denotes the orthogonal projection of $a$ onto $\left(T_{(x, y)} \mathbf{C}^{(0)}\right)^{\perp}$ for $(x, y) \in \mathbf{C}^{(0)}$. But this would imply that $\mathbf{C}_{0}^{(0)}$ is invariant under translations in the direction $a$, contradicting the fact that $\operatorname{sing} \mathbf{C}_{0}^{(0)}=\{0\}$. Thus (1) is established. We use (1) with $a=\xi$, where $Z=(\xi, \eta)$ is as in the statement of the lemma.

With $\rho_{0} \in\left(0, \frac{1}{4}\right)$ and $\varepsilon_{0}=\varepsilon_{0}\left(\rho_{0}, \mathbf{C}^{(0)}, \mathscr{M}\right)>0$ sufficiently small, (1) with $\rho=\rho_{0}$ yields

$$
\rho_{0}^{n}|\xi|^{2} \leq C \int_{M \cap B_{\rho_{0}}(Z)}\left|\xi^{\perp}\right|^{2}
$$

with $\theta=\theta\left(\mathbf{C}^{(0)}\right) \in(0,1)$. Thus by $(1.5),(3.6)$, and (3.7) we have

$$
|\xi|^{2} \rho_{0}^{n} \leq C \int_{M \cap B_{\rho_{0}}(Z)} d_{Z}^{2}+C \rho_{0}^{-2}|\xi|^{4}+C \int_{M \cap B_{1}} d^{2}
$$

But by applying Lemma 3.4 with $\eta_{Z, 1 / 2} M$ in place of $M$ and using (3.8) we obtain

$$
\rho_{0}^{-n-2+\alpha} \int_{M \cap B_{\rho_{0}}(Z)} d_{Z}^{2} \leq C \int_{M \cap B_{1}} d_{Z}^{2} \leq C \int_{M \cap B_{1}} d^{2}+C|\xi|^{2}
$$

Notice that here $C=C\left(\mathbf{C}^{(0)}, \mathscr{M}\right)$ does not depend on $\rho_{0}$. Thus combining (2) and (3) yields

$$
|\xi|^{2} \rho_{0}^{n} \leq 2 C \int_{M \cap B_{1}} d^{2}+C\left(\rho_{0}^{n+2-\alpha}+\rho_{0}^{-2}|\xi|^{2}\right)|\xi|^{2}, \quad C=C\left(\mathbf{C}^{(0)}, \mathscr{M}\right) .
$$


Since by Lemma 2.6 (see the discussion preceding the lemma) we know already that

$$
|\xi| \leq \delta\left(\varepsilon_{0}, \mathbf{C}^{(0)}, \mathscr{M}\right), \quad \text { with } \delta\left(\varepsilon, \mathbf{C}^{(0)}, \mathscr{M}\right) \downarrow 0 \text { as } \varepsilon \downarrow 0,
$$

by choosing $\rho_{0}$ suitably small (depending on $\left.\mathbf{C}^{(0)}, \mathscr{M}\right)$ from $(4)$ it follows that

$$
|\xi|^{2} \leq C \int_{M \cap B_{1}} d^{2}
$$

provided $\varepsilon_{0}=\varepsilon_{0}\left(C^{(0)}, \mathscr{M}\right)$ is sufficiently small, and by (3.8) we also have

$$
\int_{M \cap B_{1}} d_{Z}^{2} \leq 2 \int_{M \cap B_{1}} d^{2}+C|\xi|^{2}
$$

The required inequalities are thus proved.

Proof of Theorem 3.1. First select $Z=(\xi, \eta) \in B_{3 / 4}$ with $\boldsymbol{\Theta}_{M}(Z) \geq$ $\boldsymbol{\Theta}_{\mathbf{C}^{(0)}}(0)$. Note that by Lemma 3.9 we have

(1) $|\xi|^{2} \equiv \operatorname{dist}^{2}\left(Z,\{0\} \times \mathbf{R}^{m}\right) \leq C \int_{M \cap B_{1}} d^{2}, \quad C=C\left(\mathbf{C}^{(0)}, \mathscr{M}\right)$.

Also, by applying Lemma 3.4 to $\eta_{Z, 1 / 4} M$, and using (3.8), we obtain

$$
\int_{M \cap B_{1 / 4}(Z)} \frac{d_{Z}^{2}}{|X-Z|^{n+2-\alpha}} \leq C \int_{M \cap B_{1}} d^{2}
$$

where $d_{Z}(X) \equiv \operatorname{dist}\left(X, \tau_{Z} \mathbf{C}\right), \tau_{Z}$ as in (3.5). Of course this implies

$$
\int_{M \cap B_{1 / 4}(Z)} \frac{d_{Z}^{2}}{|X-Z|^{n-\alpha}} \leq C \int_{M \cap B_{1}} d^{2}
$$

and since $d_{Z}(X) \leq d(X)+|\xi|$ by (3.8), this together with (1) gives that

$$
\int_{M \cap B_{1 / 4}(Z)} \frac{d^{2}}{|X-Z|^{n-\alpha}} \leq C \int_{M \cap B_{1}} d^{2}, \quad C=C\left(\mathbf{C}^{(0)}, \mathscr{M}, \alpha\right) .
$$

Next we prove the last inequality in Theorem 3.1. Notice that by (3.5) and (3.6) we have

$$
d_{Z}((x, y)+u(x, y))=\left|u(x, y)-\xi^{\perp}\right|+\widetilde{R}
$$

where $|\widetilde{R}| \leq \tau^{-1} C|\xi|^{2}$ for $(x, y) \in U_{\tau}$, and hence (2) implies

$$
\begin{aligned}
& \int_{U_{\tau} \cap B_{1 / 4}(Z)} \frac{\left|u(x, y)-\xi^{\perp}\right|^{2}}{|X-Z|^{n+2-\alpha}} \\
& \leq C \tau^{-2}|\xi|^{4} \int_{U_{\tau} \cap B_{1 / 4}(Z)} \frac{1}{|X-Z|^{n+2-\alpha}}+C \int_{M \cap B_{1}} d^{2} .
\end{aligned}
$$


Since $|X-Z| \geq \tau$ in $U_{\tau}$, assuming (as we may) that $\varepsilon_{0} \leq \tau^{2}$, we thus deduce

$$
\begin{aligned}
\tau^{-2}|\xi|^{4} \int_{U_{\tau} \cap B_{1 / 4}(Z)} \frac{1}{|X-Z|^{n+2-\alpha}} & \leq \tau^{-4}|\xi|^{4} \int_{\mathbf{C} \cap B_{1 / 4}(Z)} \frac{1}{|X-Z|^{n-\alpha}} \leq C|\xi|^{2} \\
& \leq C \int_{M \cap B_{\gamma}} d^{2} \text { by }(1),
\end{aligned}
$$

where $C=C\left(\mathbf{C}^{(0)}, \mathscr{M}\right)$; notice that $C$ does not depend on $\tau$. Hence the last inequality in Theorem 3.1 is proved.

\section{Linear asymptotics}

Let $\mathbf{C}^{(0)}=\mathbf{C}_{0}^{(0)} \times \mathbf{R}^{m} \in \mathscr{M}$ be as in $\S 1$, let $\mathscr{L}_{\mathbf{C}^{(0)}}$ be the Jacobi-field operator of $\S 1$, and let $\Sigma_{0}=\mathbf{C}_{0}^{(0)} \cap S^{l+k-1}$. Here we consider solutions $v$ of the equation $\mathscr{L}_{\mathrm{C}^{(0)}} v=0$, subject to $L^{2}$ restrictions of the type given for $u$ in Theorem 3.1. For example, for Lemma 4.2 below we assume

$$
\int_{\mathbf{C}^{(0)} \cap B_{1}} \frac{|v-\kappa|^{2}}{r^{l+2-\alpha}}<\infty
$$

for some $\alpha \in(0,2)$, where $\kappa(x, y)=\sum_{j=1}^{l+k} \kappa_{j}(r, y) e_{j}^{\perp}$, with $\kappa_{j}$ bounded.

Our first aim here is to analyze the homogeneous degree 1 solutions of $\mathscr{L}_{\mathbf{C}^{(0)}} v=0$ subject to (4.1); the main result is as follows.

4.2. Lemma. If $v \in C^{2}\left(\mathbf{C}^{(0)} ;\left(\mathbf{C}^{(0)}\right)^{\perp}\right)$ is a homogeneous degree 1 solution of $\mathscr{L}_{\mathbf{C}^{(0)}} v=0$ on $\mathbf{C}^{(0)}$, and $v$ satisfies (4.1) for some $\alpha<2$, then, for $l \geq 2$,

$$
v(x, y) \equiv \sum_{i=1}^{l+k} y \cdot \eta_{i} e_{i}^{\perp}+r \varphi(\omega)
$$

for some $\eta_{1}, \cdots, \eta_{l+k} \in \mathbf{R}^{m}$ and some $\varphi \in C^{\infty}\left(\Sigma_{0} ;\left(\mathbf{C}^{(0)}\right)^{\perp}\right)$. If $l=1$ the same conclusion holds under the additional hypothesis that

$$
\lim _{r \downarrow 0} \frac{\partial^{2}}{\partial r \partial y^{i}} \sum_{\omega \in \Sigma_{0}} v(r \omega, y)=0
$$

for each $i=1, \cdots, l+k$, uniformly for $|y| \leq 1$.

4.3. Remarks. (1) Of course $\varphi$ must be an eigenfunction of $-L_{\Sigma_{0}}$ with eigenvalue $l-1$ if $l \geq 2$.

(2) In case $l=1$, when $\Sigma_{0}$ consists of a finite collection of points $\left\{\omega_{1}, \cdots, \omega_{N}\right\}$, the statement $\varphi \in C^{\infty}\left(\Sigma_{0} ;\left(\mathbf{C}^{(0)}\right)^{\perp}\right)$ should be taken to 
mean that $\varphi$ is any map $\Sigma_{0} \rightarrow \mathbf{R}^{n+k}$ such that $\varphi(\omega) \in\left(T_{(\omega, 0)} \mathbf{C}^{(0)}\right)^{\perp}$ at each of the points $\omega=\omega_{j}$ of $\Sigma_{0}$.

(3) No conclusion like that in Lemma 4.2 above can be drawn without the additional hypothesis if $l=1, m \geq 2$. In fact in this case if $p$ is an arbitrary $C^{\infty}\left(S^{m-1}\right)$ function with $\int_{S^{m-1}} p(\omega) d \omega=0$, then there is a homogeneous degree 1 solution $v$ of $L_{\mathbf{C}^{(0)}} v=0$ having the form $v(r \omega, y)=r \psi(y / r) e^{\perp}$, where $e \in \mathbf{R}^{1+k}$ is arbitrary, and $\psi \in C^{\infty}\left(\mathbf{R}^{m}\right)$ with $|z|^{-1} \psi(z) \sim p\left(|z|^{-1} z\right)$ as $|z| \rightarrow \infty$; more precisely,

$$
\left.|| z\right|^{-1} \psi(z)-\left.p\left(|z|^{-1} z\right)|\leq C| z\right|^{-1} \text { for }|z| \geq 1 .
$$

One can check that then the $L^{2}$ restriction (4.1) holds with $\kappa(z)=$ $p\left(|z|^{-1} z\right) e^{\perp}$. On the other hand we can easily verify (although we shall not make use of it here) that the additional hypothesis is not needed in case $l=m=1$.

We first dispense with the case $l=1$ of Lemma 4.2.

Proof of Lemma 4.2 for $l=1$. In case $l=1, \Sigma_{0}$ is a finite set of points $\left\{\omega_{1}, \cdots, \omega_{N}\right\}$ in $S^{1+k}$, so $\mathbf{C}^{(0)}$ is just a union $\bigcup_{j=1}^{N} H_{j}$, where $H_{j} \equiv\left\{\left(r \omega_{j}, y\right): r>0, y \in \mathbf{R}^{n-1}\right\}$ is an $n$-dimensional half-space in $\mathbf{R}^{n+k}$ with $\partial H_{j}=\{0\} \times \mathbf{R}^{n-1}$ for each $j=1, \cdots, N$. The equation $\mathscr{L}_{\mathbf{C}^{(0)}} v=0$ in this case is simply $\Delta v \equiv\left(\Delta v^{1}, \cdots, \Delta v^{n+k}\right)=0$, with $v=\left(v^{1}, \cdots, v^{n+k}\right)$ normal to $H_{j}$ on $H_{j}$ for each $j=1, \cdots, N$. Thus in this case $v$ is a homogeneous degree 1 function satisfying

$$
\Delta v^{i}=0 \text { on } H_{j}, \quad i=1, \cdots, n+k, j=1, \cdots, N,
$$

and

$$
\int_{0}^{1} \int_{B_{1}^{m}} r^{-3+\alpha}\left|v\left(r \omega_{j}, y\right)-\kappa\left(r \omega_{j}, y\right)\right|^{2} d y d r<\infty
$$

with

$$
\kappa\left(r \omega_{j}, y\right)=\sum_{k=1}^{1+k} \kappa^{i}(r, y) e_{i}^{\perp} \quad \text { on } H_{j}, \quad \sup \left|\kappa^{i}\right|<\infty,
$$

together with the additional hypothesis that $\lim _{r \downarrow 0} \frac{\partial^{2}}{\partial r \partial y^{i}} \sum_{j=1}^{N} v\left(r \omega_{j}, y\right)=$ 0 . By the reflection principle this means that

$$
w(r, y) \equiv \frac{\partial}{\partial y^{i}} \sum_{j=1}^{N} v\left(r \omega_{j}, y\right)
$$


extends via even relection in the $r$-variable to a homogeneous degree zero harmonic function on all of $\mathbf{R}^{n}$, and hence must be constant. Thus

$$
\sum_{j=1}^{N} v\left(r \omega_{j}, y\right) \equiv r a+\sum_{j=1}^{n-1} y^{i} b_{i}
$$

for some $a, b_{i} \in \mathbf{R}^{1+k} \times\{0\}$. For each $j=1, \cdots, N$, let $P_{j}: \mathbf{R}^{1+k} \times$ $\{0\} \rightarrow \mathbf{R}^{1+k} \times\{0\}$ denote the orthogonal projection of $\mathbf{R}^{1+k} \times\{0\}$ onto the orthogonal complement of the $n$-dimensional subspace containing the half-space $H_{j}$. (1) evidently implies

$$
\lim _{r \downarrow 0} \rho^{-3-\alpha} \int_{0}^{\rho} \int_{B_{1}^{n-1}}\left|\sum_{j=1}^{N} P_{j}(\tilde{\kappa}(r, y))-\sum_{i=1}^{n-1} y^{i} b_{i}\right|^{2}=0,
$$

where $\tilde{\kappa}(r, y)=\left(\kappa^{1}, \cdots, \kappa^{1+k}, 0\right)$, with $\kappa^{i}$ as in (1). Notice that in particular (2) implies that each $b_{i}$ is in the subspace $\left\{\sum_{j=1}^{N} P_{j}(c): c \in\right.$ $\left.\mathbf{R}^{1+k} \times\{0\}\right\}$, so in fact (2) can be written

$$
\lim _{r \downarrow 0} \rho^{-3-\alpha} \int_{0}^{\rho} \int_{B_{1}^{n-1}}\left|\sum_{j=1}^{N} P_{j}\left(\tilde{\kappa}(r, y)-\sum_{i=1}^{n-1} y^{i} c_{i}\right)\right|^{2} d y d r=0
$$

for suitable $c_{i} \in \mathbf{R}^{1+k} \times\{0\}$. But then

$$
\begin{aligned}
\lim _{\rho \downarrow 0} \rho^{-1} \int_{0}^{\rho} \int_{B_{1}^{n-1}} \sum_{j=1}^{N}\left|P_{j}\left(\tilde{\kappa}(r, y)-\sum_{i=1}^{n-1} y^{i} c_{i}\right)\right|^{2} d y d r \\
\equiv \lim _{\rho \downarrow 0} \rho^{-1} \int_{0}^{\rho} \int_{B_{1}^{n-1}}\left(\sum_{j=1}^{N} P_{j}\left(\tilde{\kappa}(r, y)-\sum_{i=1}^{n-1} y^{i} c_{i}\right)\right) \\
\cdot\left(\tilde{\kappa}(r, y)-\sum_{i=1}^{m} y^{i} c_{i}\right) d y d r=0,
\end{aligned}
$$

because $\tilde{\kappa}$ is bounded. Thus

$$
\lim _{\rho \downarrow 0} \rho^{-1} \int_{0}^{\rho} \int_{B_{1}^{n-1}}\left|P_{j}\left(\tilde{\kappa}(r, y)-\sum_{i=1}^{n-1} y^{i} c_{i}\right)\right|^{2} d y d r=0
$$

for each $j=1, \cdots, N$, and, by (1) again,

$$
\lim _{\rho \downarrow 0} \rho^{-1} \int_{0}^{\rho} \int_{B_{1}^{n-1}}\left|v\left(r \omega_{j}, y\right)-\sum_{i=1}^{n-1} y^{i} P_{j} c_{i}\right|^{2} d y d r=0
$$


for each $j=1, \cdots, N$. Since $v\left(r \omega_{j}, y\right)=\sum_{i=1}^{n-1} y^{i} P_{j} c_{i}$ is harmonic on $\left\{(r, y): r>0, y \in \mathbf{R}^{n-1}\right\}$, it follows (for example by using a Green's representation formula on the half-ball) that $v\left(r \omega_{j}, y\right)$ extends continuously to $r=0$, with boundary values $\sum_{i=1}^{n-1} y^{i} P_{j} c_{i}$. Thus we can make an odd reflection (with respect to the $r$ variable) of $v\left(r \omega_{j}, y\right)-\sum_{i=1}^{n-1} y^{i} P_{j} c_{i}$, and hence conclude, since $v$ is homogeneous of degree 1 , that

$$
v\left(r \omega_{j}, y\right)=r a_{j}+\sum_{i=1}^{n-1} y^{i} P_{j}\left(c_{i}\right), \quad j=1, \cdots, N,
$$

for some $a_{j} \in P_{j}\left(\mathbf{R}^{1+k} \times\{0\}\right)$, which is the required result.

Before we begin the proof of Lemma 4.2 for the case $l \geq 2$, we need some preliminary discussion. First note that if $\lambda_{1} \leq \lambda_{2} \cdots$ are the eigenvalues of $-\mathscr{L}_{\Sigma_{0}}$ as in $\S 1$, and $\varphi_{1}, \varphi_{2}, \cdots$ are a corresponding orthonormal set of eigenfunctions, then $v_{j}=\left\langle v, \varphi_{j}\right\rangle_{L^{2}(\Sigma)}$ satisfies the equation

$$
\frac{1}{r^{l-1}} \frac{\partial}{\partial r}\left(r^{l-1} \frac{\partial v_{j}}{\partial r}\right)+\Delta_{y} v_{j}-\lambda_{j} v_{j}=0
$$

on $\mathbf{C}^{(0)}$. Notice that if $\varphi_{j}$ (with eigenvalue 0 ) is one of the eigenfunctions of the space spanned by $e_{1}^{\perp}, \cdots, e_{l+k}^{\perp}$, then (4.1) implies that $v=v_{j}$ satisfies the bound

$$
\int_{\mathbf{C}^{(0)} \cap B_{1}} \frac{|v-\kappa(r, y)|^{2}}{r^{l+2-\alpha}}<\infty,
$$

where $\kappa(r, y)=\kappa_{j}(r, y)=\left\langle\kappa, \varphi_{j}\right\rangle_{L^{2}\left(\Sigma_{0}\right)}$, whereas if $\varphi_{j}$ is not in the subspace spanned by $e_{1}^{\perp}, \cdots, e_{l+k}^{\perp}$, then (even if the eigenvalue corresponding to $\varphi_{j}$ is zero-i.e., the same as the eigenvalue of the $\left.e_{j}^{\perp}\right) \quad v=v_{j}$ satisfies

$$
\int_{\mathbf{C}^{(0)} \cap B_{1}} \frac{|v|^{2}}{r^{l+2-\alpha}}<\infty .
$$

Thus we want to analyze real-valued homogeneous degree 1 solutions $v \in$ $C^{2}\left((0, \infty) \times \mathbf{R}^{m}\right)$ of the equation

$$
\frac{1}{r^{l-1}} \frac{\partial}{\partial r}\left(r^{l-1} \frac{\partial v}{\partial r}\right)+\Delta_{y} v-\lambda v=0
$$

subject to the restrictions (4.5), and in case $\lambda=0$ we also need to analyze solutions $v$ in the space spanned by $e_{1}^{\perp}, \cdots, e_{l+k}^{\perp}$ subject to the weaker restriction (4.4). 
Notice that any such homogeneous degree 1 solution can be expressed $v(r, y)=r v(1, y / r)$; that is, writing $\psi(z) \equiv v(1, z), z \in \mathbf{R}^{m}$, we have

$$
v(r, y)=r \psi(y / r),
$$

where $\psi \in C^{\infty}\left(\mathbf{R}^{m}\right)$. By direct computation (4.6) is equivalent to the linear elliptic equation

$$
\Delta \psi+\sum_{i, j=1}^{m} z^{i} z^{j} D_{i} D_{j} \psi-(l-1) \sum_{i=1}^{m} z^{i} D_{i} \psi+(l-1-\lambda) \psi=0,
$$

and the $L^{2}$ restrictions (4.5), (4.4) are easily seen to imply

$$
\int_{1}^{\infty} r^{-1-\alpha} \int_{S^{m-1}}|\psi(r \omega)|^{2} d \omega d r<\infty
$$

and

$$
\int_{1}^{\infty} r^{1-\alpha} \int_{S^{m-1}}\left|\frac{\psi(r \omega)}{r}-\kappa(r \omega)\right|^{2} d \omega d r<\infty,
$$

where $\sup _{(0, \infty) \times \mathbf{R}^{m}}|\kappa|<\infty$.

In view of the above discussion, Lemma 4.2 for $l \geq 2$ is now evidently a direct consequence of the following lemma.

4.10. Lemma. (i) Suppose $l \geq 2$ and $\lambda \in \mathbf{R}$. If $\psi \in C^{\infty}\left(\mathbf{R}^{m}\right)$ satisfies (4.7) and (4.8), then $\psi \equiv 0$ for $l-1-\lambda \neq 0$ and $\psi \equiv$ const. for $l-1-\lambda=0$.

(ii) Suppose $l \geq 2$ and $\lambda=0$. If $\psi \in C^{\infty}\left(\mathbf{R}^{m}\right)$ satisfies (4.7) and (4.9), then $\psi(z) \equiv a \cdot z$ for some $a \in \mathbf{R}^{m}$.

Proof. We first consider $\lambda \geq 0$ and note that each partial derivative $v=D_{j} \psi$ satisfies the equation

$$
\sum_{i, j=1}^{m}\left(\delta_{i j}+z^{i} z^{j}\right) D_{i} D_{j} v-(l-3) \sum_{i=1}^{m} z^{i} D_{i} v-\lambda v=0,
$$

as one easily checks by direct differentiation in (4.7). We would like to assert that, with the notation $A_{\sigma, \rho}=B_{\rho}^{m}(0) \backslash B_{\sigma}^{m}(0)$ for any $0<\sigma<\rho$,

$$
\sup _{A_{\rho / 2, \rho}}|D \psi| \leq C \rho^{-1} \sup _{A_{\rho / 4}, 2 \rho}|\psi|, \quad \rho \geq 1,
$$

but this is not in general true for solutions of (4.7) except in the case $m=$ 1 . On the other hand if $m \geq 2$, then for any eigenfunction $\varphi_{j}$ of $-\Delta_{S^{m-1}}$ corresponding to a homogeneous degree $j$ harmonic polynomial on $\mathbf{R}^{m}$, 
we can write $\psi_{j}(z)=\gamma_{j}(r) \varphi_{j}(\omega)$, where $\gamma_{j}(r)=\int_{S^{m-1}} \psi(r \omega) \varphi_{j}(\omega) d \omega$ satisfies the ODE

$$
\left(1+r^{2}\right) \gamma_{j}^{\prime \prime}+\left(\frac{m-1}{r}-(l-1) r\right) \gamma_{j}^{\prime}-\left(\frac{j(j+m-2)}{r^{2}}+\lambda-l+1\right) \gamma_{j}=0
$$

on $(0, \infty)$ and remains bounded as $r \downarrow 0$. Notice that then $\psi_{j}$ is still a solution of (4.7) on $\mathbf{R}^{m} \backslash\{0\}$. In fact since $\psi_{j}$ is bounded near 0 , it extends across $z=0$ to give a $C^{\infty}\left(\mathbf{R}^{m}\right)$ solution; furthermore by using elementary ODE estimates for $\gamma_{j}$, we easily check that in fact (2) does hold (with constant $C$ depending on $j$ ) with $\psi_{j}$ in place of $\psi$. Also, $\psi_{j}$ certainly satisfies (4.8) (respectively (4.9)) if $\psi$ does, and if we establish the lemma with each such $\psi_{j}$, then the lemma also follows for $\psi$. In other words, we can without loss of generality assume that the inequality (2) does hold.

Now if $m \geq 2$, we introduce spherical coordinates $z=r \omega, r=|z|$, $\omega=|z|^{-1}$ in $\mathbf{R}^{m} \backslash\{0\}$, and note that (1) can be written in the form

$$
\frac{g}{r^{2}\left(1+r^{2}\right)} \Delta_{S^{m-1}} v+\frac{\partial}{\partial r}\left(g \frac{\partial v}{\partial r}\right)-\frac{\lambda g}{1+r^{2}} v=0
$$

where $g=r^{m-1}\left(1+r^{2}\right)^{-(l+m) / 2+2}$. Since $(1 / r) \nabla_{S^{m-1}} v$ is bounded as $r \downarrow 0$, this can be written in weak form

$$
\begin{array}{r}
\int_{0}^{\infty} \int_{S^{m-1}} \frac{g}{r^{2}\left(1+r^{2}\right)} \nabla^{S^{m-1}} v \cdot \nabla^{S^{m-1}} \zeta+g \frac{\partial v}{\partial r} \frac{\partial \zeta}{\partial r}+\frac{\lambda g}{1+r^{2}} v \zeta=0 \\
\forall \zeta \in C_{c}^{\infty}\left(\mathbf{R}^{m}\right) .
\end{array}
$$

Now replace $\zeta$ by $v \zeta^{2}$, where $\zeta=\zeta(r)$ is a $C^{\infty}(0, \infty)$ function which is constant in a neighborhood of $r=0$ and vanishes identically for sufficiently large $r$. Then after an application of Cauchy's inequality (3) implies

$$
\begin{aligned}
& \int_{0}^{\infty} \int_{S^{m-1}} g \zeta^{2}\left(\left(1+r^{2}\right)^{-1}\left(r^{-2}\left|\nabla^{S^{m-1}} v\right|^{2}+\lambda v^{2}\right)+\left(\frac{\partial v}{\partial r}\right)^{2}\right) d \omega d r \\
& \quad \leq C \int_{0}^{\infty} g\left(\zeta^{\prime}\right)^{2} \int_{S^{m-1}} v^{2} d \omega d r .
\end{aligned}
$$

Now we are going to use the "logarithmic cut-off trick". Let $\rho \geq 2$ be arbitrary, and let $\zeta(r)=\max \left\{2-\left(\log r_{\rho}\right) /(\log \rho), 0\right\}$, where $r_{\rho}=\max \{r, \rho\}$. Notice that then $\zeta \equiv 1$ on $[0, \rho], \zeta \equiv 0$ on $\left(\rho^{2}, \infty\right)$, and $\left(\zeta^{\prime}\right)^{2} \leq$ $r^{-2}(\log \rho)^{-2}$ on $\left(\rho, \rho^{2}\right)$. Thus using this choice of $\zeta$ in (4), noting that 
$g \leq r^{3-l}$, and using also (2), we obtain

$$
\begin{aligned}
\int_{0}^{\rho} g & \int_{S^{m-1}}\left(\left(1+r^{2}\right)^{-1}\left(r^{-2}\left|\nabla^{S^{m-1}} v\right|^{2}+\lambda v^{2}\right)+\left(\frac{\partial v}{\partial r}\right)^{2}\right) d \omega d r \\
& \leq \frac{C}{(\log \rho)^{2}} \int_{\rho / 2}^{2 \rho^{2}} r^{-1-l} \int_{S^{m-1}} \psi^{2}(r \omega) d \omega d r \\
& \left.\leq \frac{C}{(\log \rho)^{2}} \int_{\rho / 2}^{2 \rho^{2}} r^{-3} \int_{S^{m-1}} \psi^{2}(r \omega) d \omega d r \quad \text { (since } l \geq 2\right) \\
& \leq \frac{2 C}{(\log \rho)^{2}} \int_{\rho / 2}^{2 \rho^{2}} r^{-1} \int_{S^{m-1}}\left|r^{-1} \psi(r \omega)-\kappa\right|^{2} d \omega d r+6 C \frac{\kappa_{0}}{\log \rho}
\end{aligned}
$$

where $\kappa_{0}$ is any upper bound for $|\kappa|$. Letting $\rho \uparrow \infty$, and using (4.9), we see that this implies $\nabla v \equiv 0$ in case $\lambda=0$ and $v \equiv 0$ in case $\lambda>0$. Notice that in case $\lambda=0$ we get $\psi \equiv a \cdot z+b$ for fixed $a \in \mathbf{R}^{m}, b \in \mathbf{R}$. If the stronger estimate (4.8) holds, then evidently $a=0$ and $\psi \equiv b$. So the lemma is proved if $m \geq 2$ and $\lambda \geq 0$.

For $m=1, l \geq 2$, and $\lambda \geq 0$, the argument is similar: We can write (1) in the form

$$
\frac{d}{d z}\left(g v^{\prime}(z)\right)-\frac{\lambda g}{1+z^{2}} v=0
$$

where $g=\left(1+z^{2}\right)-(l-f 3) / 2$, which in weak form is

$$
\int_{\mathbf{R}} g v^{\prime} \zeta^{\prime}+\frac{\lambda g}{1+z^{2}} v \zeta=0, \quad \forall \zeta \in C_{c}^{\infty}(\mathbf{R}) .
$$

Replacing $\zeta$ by $\zeta^{2} v$, where $\zeta=\zeta(|z|)$ is the same logarithmic cut-off function as before, we get (as in the case $m \geq 2$ )

$$
\int_{-\rho}^{\rho}\left(g\left(v^{\prime}\right)^{2}+\frac{\lambda g}{1+z^{2}} v^{2}\right) \leq \frac{C}{(\log \rho)^{2}} \int_{\rho / 2<|z|<2 \rho^{2}}|z|^{-3} v^{2} d z,
$$

and the proof is concluded essentially as before.

For $m=1, l \geq 2$, and $\lambda<0$ we note that the equation for $\psi$ can be written

$$
\left(1+z^{2}\right) \psi^{\prime \prime}-(l-1) z \psi^{\prime}+(l-1-\lambda) \psi=0,
$$

and by using series expansions near $\pm \infty$ one easily checks that any solution $\psi$ which is not identically zero satisfies

$$
\liminf _{\rho \rightarrow \infty} \rho^{-1-2 q_{-}} \int_{\rho / 2<|z|<\rho}|\psi(z)|^{2}>0,
$$


where $q_{-}=\operatorname{Re}\left(1+(l-2) / 2-\sqrt{((l-2) / 2)^{2}+\lambda}\right)$. Since $\lambda<0$ and $l \geq 2$, clearly we have $q_{-} \geq 1$, so that (6) contradicts the growth restriction (4.8).

For $m \geq 2, l \geq 2$, and $\lambda<0$ we let $\gamma_{j}(r), \varphi_{j}$ be as described above, and note that $\gamma_{j}$ satisfies the ODE

$$
\left(1+r^{2}\right) \gamma_{j}^{\prime \prime}+\left(\frac{m-1}{r}-(l-1) r\right) \gamma_{j}^{\prime}-\left(\frac{j(j+m-2)}{r^{2}}+\lambda-l+1\right) \gamma_{j}=0 \text {, }
$$

and again by taking series expansion near $\infty$, we see that any solution $\gamma_{j}$ which is not identically zero satisfies

$$
\liminf _{\rho \rightarrow \infty} \rho^{-1-2 q_{-}} \int_{\rho / 2}^{\rho}\left|\gamma_{j}(r)\right|^{2}>0
$$

and again this contradicts the growth restriction (4.8), because $q_{-} \geq 1$. Thus the proof of Lemma 4.10, and hence the proof of Lemma 4.2, is complete.

Now we show that Lemma 4.2 leads to decay estimates for an appropriate class of solutions of the equation $\mathscr{L}_{\mathbf{C}^{(0)} v} v=0$ on $\mathbf{C}^{(0)} \cap B_{1}$. We here need to consider solutions which are orthogonal on $C^{(0)} \cap B_{1}$ to the solutions of Lemma 4.2. Thus we assume that

$$
\int_{\mathbf{C}^{(0)} \cap B_{1}} v(r \omega, y) r \varphi(\omega)=0, \quad \int_{\mathbf{C}_{0}^{(0)} \cap B_{1}} v(r \omega, y) y^{i}\left(e_{j}\right)^{\perp}=0,
$$

for each solution $w=r \varphi(\omega)$ of $\mathscr{L}_{\mathbf{C}^{(0)}}=0$ and each $i=1, \cdots, m$, $j=1, \cdots, l+k$, where $\left(e_{j}\right)^{\perp}$ is the orthogonal projection of the unit vector $e_{j}$ onto $\left(T_{\omega} \mathrm{C}^{(0)}\right)^{\perp}$. (Notice that then $\left(e_{j}\right)^{\perp}$ is a function of $\omega$ alone; it does not depend on $r, y$.) Notice that (4.11) is equivalent to

$$
\min _{\psi \in \mathscr{L}} \int_{\mathbf{C}^{(0)} \cap B_{1}}|v-\psi|^{2}=\int_{\mathbf{C}^{(0)} \cap B_{1}}|v|^{2},
$$

where $\mathscr{L}$ is the linear space spanned by the homogeneous degree one solutions of Lemma 4.2 ; that is, $\mathscr{L}$ is spanned by the solutions $y^{i}\left(e_{j}\right)^{\perp}$, $i=1, \cdots, m, j=1, \cdots, l+k, r \varphi(\omega)$.

Then we have:

4.12. Lemma. Let $\beta_{1}, \beta_{2} \geq 1, \alpha \in(0,2)$ be given constants, and suppose $v \in C^{\infty}\left(\mathbf{C}^{(0)} \cap \bar{B}_{1} ;\left(\mathbf{C}^{(0)}\right)^{\perp}\right)$ is a solution of the equation $\mathscr{L}_{\mathbf{C}^{(0)}} v=0$ on $\mathrm{C} \cap B_{1}$ satisfying (4.11) and also

$$
\int_{\mathbf{C}^{(0)} \cap B_{1 / 2}} \frac{|v-\kappa|^{2}}{r^{l+2-\alpha}} \leq \beta_{1} \int_{\mathbf{C}^{(0)} \cap B_{1}}|v|^{2}
$$


where $\kappa(r \omega, y)=\sum_{i=1}^{l+k} \kappa_{i}(r, y) e_{i}^{\perp}$, with $\sup \sum_{j=1}^{l+k}\left|\kappa_{j}\right|^{2} \leq \beta_{2} \int_{\mathbf{C}^{(0)} \cap B_{1}}|v|^{2}$, and $e_{i}^{\perp}=e_{i}^{\perp}(\omega)$ denoting the orthogonal projection of $e_{i}$ onto $\left(T_{(\omega, 0)} \mathbf{C}^{(0)}\right)^{\perp}, r=|x|, \omega=|x|^{-1} x$. Then, for $l \geq 2$,

$$
\int_{\mathbf{C}^{(0)} \cap B_{1} \backslash B_{1 / 4}}\left(\frac{\partial(v / R)}{\partial R}\right)^{2} \geq \beta^{-1} \int_{\mathbf{C}^{(0)} \cap B_{1}}|v|^{2},
$$

with $\beta=\beta\left(\alpha, \beta_{1}, \beta_{2}, \mathbf{C}^{(0)}\right) \geq 1$. The same holds in case $l=1$ subject to the additional assumption

$$
\lim _{r \downarrow 0} \frac{\partial^{2}}{\partial r \partial y^{i}} \sum_{\omega \in \Sigma_{0}} v(r \omega, y)=0, \quad i=1, \cdots, l+k,
$$

uniformly for $|y| \leq \frac{1}{4}$.

Proof. The proof is based on the results of Lemma 4.2. Suppose the lemma is false. Then there is a sequence $v_{j}$ of $C^{\infty}$ solutions of $\mathscr{L}_{\mathbf{C}^{(0)}} v=0$ in $\mathbf{C}^{(0)} \cap B_{1}$, with each $v_{j}$ satisfying (4.11) and the $L^{2}$-bound $(*)$ in the statement of the lemma, but such that

$$
\int_{\mathbf{C}^{(0)} \cap B_{1} \backslash B_{1 / 4}}\left(\frac{\partial\left(v_{j} / R\right)}{\partial R}\right)^{2} \leq \varepsilon_{j} \int_{\mathbf{C}^{(0)} \cap B_{1}}\left|v_{j}\right|^{3},
$$

where $\varepsilon_{j} \rightarrow 0$ as $j \rightarrow \infty$. Notice that $(*) \Rightarrow$, for each $\delta>0$,

$$
\int_{\mathbf{C}^{(0)} \cap B_{1 / 2} \cap\left(B_{\delta}^{l+k} \times \mathbf{R}^{m}\right)}\left|v_{j}\right|^{2} \leq C \delta^{l-\alpha} \int_{\mathbf{C}^{(0)} \cap B_{1}}\left|v_{j}\right|^{2} .
$$

Notice also that by the usual $L^{2}$ theory we have fixed bounds on all the derivatives of $\left\|v_{j}\right\|_{L^{2}}^{-1} v_{j}$ on compact subsets of $\mathbf{C}^{(0)} \cap B_{1}$. Then by (2) we have a subsequence $w_{j}$ of $\left\|v_{j}\right\|_{L^{2}}^{-1} v_{j}$ which converges strongly in the $L^{2}$ norm on $\mathbf{C}^{(0)} \cap B_{1 / 2}$, and furthermore the limit is with respect to the $C^{2}$-norm on fixed compact subsets $K \subset \mathbf{C}^{(0)} \cap B_{1 / 2}$. Let the limit function be $v$; of course $v$ satisfies $\mathscr{L}_{\mathbf{C}^{(0)}} v=0$ on $\mathbf{C}^{(0)} \cap B_{1 / 2}$, and, by virtue of $(*)$, we have

$$
\int_{\mathbf{C}^{(0)} \cap B_{1 / 2}} \frac{|v-\kappa|^{2}}{r^{l+2-\alpha}}<\infty, \quad \kappa(x, y)=\sum_{j=1}^{l+k} \kappa_{j}(r, y) e_{j}^{\perp}, \quad \sup \sum_{j=1}^{l+k}\left|\kappa_{j}\right|<\infty,
$$

and, by (1), $v$ extends to be a homogeneous of degree 1 on $\mathbf{C}^{(0)}$. But now, in case $l \geq 2$, Lemma 4.2 implies that

$$
v(x, y) \equiv \sum_{i=1}^{l+k} y \cdot \eta_{i} e_{i}^{\perp}+r \varphi(\omega),
$$


and this contradicts (4.11) unless $v \equiv 0$. Thus we deduce that $w_{j}$ converges uniformly to zero on compact subsets of $C^{(0)} \cap B_{1 / 2}$, which (by direct radial integration, keeping in mind that $\left\|w_{j}\right\|_{L^{2}\left(B_{1}\right)}=1$ and that the $L^{2}$ norm of $w_{j}$ over $\mathbf{C}^{(0)} \cap B_{1 / 2} \cap B_{\delta}^{l+k} \times \mathbf{R}^{m}$ is small by $\left.(*)\right)$, we deduce that, contrary to $(1)$,

$$
\int_{\mathbf{C}^{(0)} \cap B_{1}}\left(\frac{\partial\left(w_{j} / R\right)}{\partial R}\right)^{2} \geq C^{-1}
$$

with $C \geq 1$ independent of $j$.

In case $l=1, \mathscr{L}_{\mathbf{C}^{(0)}}$ is just the Laplacian on $\mathbf{C}^{(0)}$ (see the discussion in the proof of Lemma 4.2), and the given conditions guarantee that each of the functions $v_{j}$ is such that

$$
w_{j}^{(p)}(r, y) \equiv \sum_{\omega \in \Sigma_{0}} \frac{\partial}{\partial y^{p}} v_{j}(r \omega, y)
$$

extends to a harmonic function on $B_{1 / 4}^{n}(0)$ which is even in the $r$-variable, and hence the limit function $v$ has the property that $\sum_{\omega \in \Sigma_{0}} \partial v(r \omega, y) / \partial y^{p}$ extends, via even reflection in the $r$-variable to a harmonic function on $B_{1 / 4}^{n}(0)$. Thus $v$ satisfies the conditions of Lemma 4.2 for $l=1$, and the proof can be completed as for the case $l \geq 2$.

For the next lemma, we need one further piece of notation. As in $\left(4.11^{\prime}\right)$, let $\mathscr{L}$ be the space of solutions described in Lemma 4.2, and for each $\rho \in(0,1]$ let

$$
v_{\rho}=v-\psi_{\rho},
$$

where $\psi_{\rho} \in \mathscr{L}$ is selected such that

$$
\int_{\mathbf{C}^{(0)} \cap B_{\rho}}\left|v-\psi_{\rho}\right|^{2}=\min _{\psi \in \mathscr{L}} \int_{\mathbf{C}^{(0)} \cap B_{\rho}}|v-\psi|^{2} .
$$

(Notice that then (4.11) holds with $B_{\rho}$ in place of $B_{1}$.) Then we have the following:

4.14. Lemma. Suppose $\theta \in\left(0, \frac{1}{8}\right), \beta_{1}, \beta_{2}>0, \alpha \in(0,2)$, that $v$ is a solution of $\mathscr{L}_{\mathbf{C}^{(0)}} v=0$ on $\mathbf{C}^{(0)} \cap B_{1}$, and that, for each $\rho \in\left[\theta, \frac{1}{4}\right], v$ also satisfies

$$
\begin{gathered}
\rho^{l+2-\alpha} \int_{\mathbf{C}^{(0)} \cap B_{\rho / 2}} \frac{\left|v_{\rho}-\kappa_{\rho}\right|^{2}}{r^{l+2-\alpha}} \leq \beta_{1} \int_{\mathbf{C}^{(0)} \cap B_{\rho}}\left|v_{\rho}\right|^{2}, \\
\int_{\mathbf{C}^{(0)} \cap B_{\rho / 4}} R^{2-n}\left(\frac{\partial(v / R)}{\partial R}\right)^{2} \leq \beta_{2} \rho^{-n-2} \int_{\mathbf{C}^{(0)} \cap B_{\rho}}\left|v_{\rho}\right|^{2},
\end{gathered}
$$


where

$$
\kappa_{\rho}(x, y)=\sum_{i=1}^{l+k} \kappa_{\rho}^{i}(r, y) e_{i}^{\perp} \text { and } \sup _{B_{\rho}}\left|\kappa_{\rho}^{i}\right|^{2} \leq \beta_{2} \rho^{-n} \int_{\mathbf{C}^{(0)} \cap B_{\rho}}\left|v_{\rho}\right|^{2} .
$$

Suppose also that in case $l=1$ we have the additional condition that

$$
\lim _{r \downarrow 0} \frac{\partial^{2}}{\partial r \partial y^{i}} \sum_{\omega \in \Sigma_{0}} v(r \omega, y)=0
$$

uniformly for $|y| \leq \frac{1}{4}$ and $i=1, \cdots, m$. Then

$$
\theta^{-n-2} \int_{\mathbf{C}^{(0)} \cap B_{\theta}}\left|v_{\theta}\right|^{2} \leq C \theta^{\mu} \int_{\mathbf{C}^{(0)} \cap B_{1}}\left|v_{1}\right|^{2},
$$

where the notation is as in (4.13) and $\mu=\mu\left(\mathbf{C}^{(0)}, \beta_{1}, \beta_{2}, \alpha\right) \in(0,1)$, $C=C\left(\mathbf{C}^{(0)}, \beta_{1}, \beta_{2}, \alpha\right)>0$. In particular, $\mu, C$ do not depend on $\theta$.

Remark. Of course since $v_{\rho}=v-\psi_{\rho}$, with $\psi_{\rho}$ homogeneous of degree one (in fact with $\psi_{\rho} \in \mathscr{L}$ ), we have

$$
\frac{\partial(v / R)}{\partial R}=\frac{\partial\left(v_{\rho} / R\right)}{\partial R} \text { for each } \rho .
$$

Proof. By Lemma 4.12 for $l \geq 1$ we have

$$
\int_{\mathbf{C}^{(0)} \cap B_{\rho} \backslash B_{\rho / 4}} R^{2-n}\left(\frac{\partial(v / R)}{\partial R}\right)^{2} \geq \beta^{-1} \rho^{-n-2} \int_{\mathbf{C}^{(0)} \cap B_{\rho}}\left|v_{\rho}\right|^{2},
$$

with $\beta$ depending only on $\alpha, \beta_{1}, \beta_{2}, \mathbf{C}^{(0)}$. Notice that then by (ii)

$$
\int_{\mathbf{C}^{(0)} \cap B_{\rho} \backslash B_{\rho / 4}} R^{2-n}\left(\frac{\partial(v / R)}{\partial R}\right)^{2} \geq\left(\beta \beta_{2}\right)^{-1} \int_{\mathbf{C}^{(0)} \cap B_{\rho / 4}} R^{2-n}\left(\frac{\partial(v / R)}{\partial R}\right)^{2},
$$

and hence, by adding $\int_{C^{(0)} \cap B_{\rho / 4}} R^{2-n}(\partial(v / R) / \partial R)^{2}$ to each side of the last inequality ("hole filling"), we have

$$
\int_{\mathbf{C}^{(0)} \cap B_{\rho / 4}} R^{2-n}\left(\frac{\partial(v / R)}{\partial R}\right)^{2} \leq \gamma \int_{\mathbf{C}^{(0)} \cap B_{\rho}} R^{2-n}\left(\frac{\partial(v / R)}{\partial R}\right)^{2},
$$

where $\gamma \in(0,1)$ is a fixed constant determined by $\beta_{1}, \beta_{2}, \mathbf{C}^{(0)}$. Starting with $\rho=\frac{1}{4}$, this can be iterated $\nu$ times, where $\nu \geq 1$ is such that $\theta \in\left(4^{-\nu-1}, 4^{-\nu}\right]$, thus giving

$$
\int_{\mathbf{C}^{(0)} \cap B_{\theta}} R^{2-n}\left(\frac{\partial(v / R)}{\partial R}\right)^{2} \leq \theta^{\mu} \int_{\mathbf{C}^{(0)} \cap B_{1 / 4}} R^{2-n}\left(\frac{\partial(v / R)}{\partial R}\right)^{2},
$$


with $\mu$ such that $\theta^{\mu}=\gamma$. Hence the proof is completed by using (ii) with $\rho=1$ and (1) with $\rho=\theta$.

\section{Proofs of Lemma 1 and Theorem 1}

All constants $C$ in this section are understood to depend only on $\mathbf{C}^{(0)}$, , unless otherwise explicitly indicated.

5.1. Proof of Lemma 1. Let $\theta \in\left(0, \frac{1}{4}\right)$ be given, let $0<\varepsilon_{j} \leq \delta_{j} \downarrow 0$ be arbitrary, and let $M_{j} \in \mathscr{N}_{\varepsilon_{j}}\left(\mathbf{C}^{(0)}\right)$ and $\mathbf{C}_{j} \in \tilde{\mathscr{C}}_{\varepsilon_{j}}\left(\mathbf{C}^{(0)}\right)$ be such that the alternative (i) of Lemma 1 is false (so $(* *)$ of Remark 1.14 is true) with $M_{j}, \mathrm{C}_{j}$ in place of $M, \mathrm{C}$ and $\varepsilon_{j}, \delta_{j}$ in place of $\varepsilon_{0}, \delta_{0}$ respectively.

We aim to prove that we can find $\gamma=\gamma\left(\mathbf{C}^{(0)}, \mathscr{M}, \theta\right)$ (independent of $j$ ) and $\widetilde{\mathbf{C}}_{j} \in \widetilde{\mathscr{C}}_{\gamma \varepsilon_{j}}\left(\mathbf{C}^{(0)}\right)$ such that for infinitely many $j$

$$
\theta^{-n-2} \int_{M_{j} \cap B_{\theta}} \tilde{d}_{j}^{2} \leq C \theta^{\alpha} \int_{M_{j} \cap B_{1}} d_{j}^{2},
$$

with $C=C\left(\mathbf{C}^{(0)}, \mathscr{M}\right)$ independent of $\theta$, where $d_{j}(X)=\operatorname{dist}\left(X, \mathbf{C}_{j}\right)$, and $\tilde{d}_{j}(X)=\operatorname{dist}\left(X, \widetilde{\mathbf{C}}_{j}\right)$ for $X \in M_{j}$. In view of the arbitrariness of the sequences this will establish the lemma. Now by definition of $\tilde{\mathscr{C}}_{\varepsilon_{j}}\left(\mathbf{C}^{(0)}\right)$ (see Definition 1.12), we can find $q_{j}=\exp A_{j}, A_{j} \in \mathscr{S}$ as in Definition 1.12 with $\left|A_{j}\right| \leq \varepsilon_{j}$ and $q_{j}^{-1} \mathbf{C}_{j} \in \mathscr{C}_{\varepsilon_{j}}\left(\mathbf{C}^{(0)}\right)$.

Let $\beta_{0}=\beta_{0}\left(\mathbf{C}^{(0)}\right)$ be as in Corollary 3.2 , and let $\tau_{j} \downarrow 0$ sufficiently slowly (depending on $\theta$ ) to ensure that the conclusions of Lemma 2.6 hold (for $j$ sufficiently large) with $\beta=\beta_{0}, \gamma=\frac{3}{4}$ and with $q_{j}^{-1} \mathbf{C}_{j}, \tau_{j}, \varepsilon_{j}$, $\eta_{0, \rho} q_{j}^{-1} M_{j}$ in place of $\mathbf{C}, \tau, \varepsilon_{0}, M$ respectively, for all $\rho \in[\theta, 1]$. Then Lemma 2.6 gives $u_{j} \in C^{2}\left(U_{j} ;\left(q_{j}^{-1} \mathbf{C}_{j}\right)^{\perp}\right)$, where $U_{j} \equiv\{(x, y) \in$ $\left.q_{j}^{-1} \mathbf{C}_{j} \cap B_{3 / 4}:|x| \geq \tau_{j}\right\}$ and

$$
q_{j}^{-1} M_{j} \cap B_{3 / 4} \backslash\left\{(x, y):|x| \geq \tau_{j}\right\} \subset \text { graph } u_{j} \subset q_{j}^{-1} M_{j},
$$

and such that (for sufficiently large $j$ ) the estimates of Theorem 3.1 and Corollary 3.2 hold with $u_{j}, q_{j}^{-1} M_{j}, q_{j}^{-1} \mathbf{C}_{j}, \tau_{j}, \varepsilon_{j}$ in place of $u, M$, $\mathrm{C}, \tau, \varepsilon_{0}$ respectively. We also agree to choose $\tau_{j}$ to converge to zero at a slower rate than $\left(\int_{M_{j} \cap B_{1}} d_{j}^{2}\right)^{1 / 2}$; thus

$$
\lim \tau_{j}^{-2} \int_{M_{j} \cap B_{1}} d_{j}^{2}=0 .
$$


Now let $\rho \in\left[\theta, \frac{1}{2}\right]$. The estimates of Theorem 3.1 and Corollary 3.2 (applied to $\eta_{0, \rho} q_{j}^{-1} M_{j}$ with $\alpha=\frac{1}{2}$ ) tell us that, for $j$ sufficiently large,

$$
\rho^{l+3 / 2} \int_{U_{j} \cap B_{\rho / 2}} \frac{\left|u_{j}-\kappa_{\rho, j}(r \omega, u)\right|^{2}}{r_{\delta}^{l+3 / 2}} \leq C \int_{M_{j} \cap B_{\rho}} d_{j}^{2} \text { for } \delta \geq 2 \theta^{-1} \delta_{j}
$$

with $\kappa_{\rho, j}(r \omega, y)=\sum_{i=1}^{l+k} \kappa_{\rho, j}^{i}(r, y) e_{i}^{\perp}$,

$$
\begin{gathered}
\sup _{U_{j} \cap B_{\rho}} \sum_{i=1}^{l+k}\left(\kappa_{\rho, j}\right)^{2} \leq C \rho^{-n} \int_{M_{j} \cap B_{\rho}} d_{j}^{2}, \\
\rho^{l-1 / 2} \int_{U_{j} \cap B_{\rho / 2}} \frac{\left|u_{j}\right|^{2}}{r_{\delta}^{l-1 / 2}}, \quad \rho^{l-1 / 2} \int_{M_{j} \cap B_{\rho / 2}} \frac{d_{j}^{2}}{r_{\delta}^{l-1 / 2}} \leq C \int_{M_{j} \cap B_{\rho}} d_{j}^{2} \\
\text { for } \delta \geq 2 \theta^{-1} \delta_{j},
\end{gathered}
$$

and

$$
\int_{U_{j} \cap B_{\rho / 2}} R^{2-n}\left(\frac{\partial\left(u_{j} / R\right)}{\partial R}\right)^{2} \leq C \rho^{-n-2} \int_{M_{j} \cap B_{\rho}} d_{j}^{2} .
$$

Next, let

$$
\beta_{j}=\left(\int_{M_{j} \cap B_{1}} d_{j}^{2}\right)^{1 / 2}, \quad v_{j}=\beta_{j}^{-1} u_{j}
$$

For each compact $K \subset B_{1} \backslash\left(\{0\} \times \mathbf{R}^{m}\right)$, by Lemma 2.6 and standard elliptic estimates [12] we know that

$$
\sup _{K \cap C_{j}}\left|\nabla^{i} v_{j}\right| \leq C, \quad i \leq 3,
$$

for sufficiently large $j$ depending on $K$, where $C=C\left(\mathbf{C}^{(0)}, K\right)$.

Since $q_{j}^{-1} \mathbf{C}_{j} \in \mathscr{C}_{\varepsilon_{j}}\left(\mathbf{C}^{(0)}\right)$ with $\varepsilon_{j} \downarrow 0$, by (2.2) we have, for a suitable sequence $\tau_{j}$ (which, by a new choice of our original sequence if necessary, we may take to be the same as the original sequence $\tau_{j}$ ),

$$
q_{j}^{-1} \mathbf{C}_{j} \cap B_{1} \backslash\left(B_{\tau_{j}}^{l+k} \times \mathbf{R}^{m}\right) \subset \operatorname{graph} \psi_{j},
$$

where $\psi_{j} \in C^{2}\left(\mathbf{C}^{(0)} \cap B_{1} \backslash\left(B_{\tau_{j} / 2}^{l+k} \times \mathbf{R}^{m}\right) ;\left(\mathbf{C}^{(0)}\right)^{\perp}\right)$ with $\left|\psi_{j}\right|_{C^{2}} \leq C \varepsilon_{j} \rightarrow 0$.

So now (8) and (9) imply that $v_{j}\left(x+\psi_{j}(x)\right)$ converges, on each compact subset of $\mathbf{C}^{(0)} \cap B_{1}$, in the $C^{2}$-norm to a limit function $v \in C^{2}\left(\mathbf{C}^{(0)} \cap B_{1}\right)$, with $\mathscr{L}_{\mathbf{C}^{(0)}} v=0$. Also the estimate (5) for $u_{j}$ then evidently guarantees 
that the convergence is strong convergence with respect to the $L^{2}$-norm on $\mathbf{C}^{(0)} \cap B_{\rho}$ for each $\rho<\frac{1}{4}$, because (5) tells us in particular that

$$
\rho^{l-1 / 2} \int_{U_{j} \cap B_{\rho / 2} \cap\left(B_{\delta}^{l+k} \times \mathbf{R}^{m}\right)}\left|v_{j}\right|^{2} \leq \delta^{l-1 / 2}, \quad \delta \geq C \delta_{j}, \quad \delta<\rho<\frac{1}{2} .
$$

In view of this, the remaining inequalities above yield directly

$$
\rho^{l+3 / 2} \int_{\mathbf{C}^{(0)} \cap B_{\rho / 2}} \frac{\left|v-\kappa_{\rho}\right|^{2}}{r^{l+3 / 2}} \leq C \int_{\mathbf{C}^{(0)} \cap B_{\rho}}|v|^{2},
$$

where $\kappa_{\rho}(r \omega, y)=\sum_{i=1}^{l+\kappa} \kappa_{\rho}^{i}(r, y) e_{i}^{\perp}$, with

$$
\sup _{\mathbf{C}^{(0)} \cap B_{\rho}} \sum_{i=1}^{l+k}\left(\kappa_{\rho}^{i}\right)^{2} \leq C \rho^{-n} \int_{\mathbf{C}^{(0)} \cap B_{\rho}}|v|^{2},
$$

and

$$
\int_{\mathbf{C}^{(0)} \cap B_{\rho / 2}} R^{2-n}\left(\frac{\partial(v / R)}{\partial R}\right)^{2} \leq C \rho^{-n-2} \int_{\mathbf{C}^{(0)} \cap B_{\rho}}|v|^{2} .
$$

We emphasize that in the last three inequalities $C$ does not depend on $\theta$.

Notice also that (5) additionally implies that

$$
\rho^{l-1 / 2} \int_{M_{j} \cap B_{\rho / 2} \cap\left(B_{\delta_{\rho}}^{l+k} \times \mathbf{R}^{m}\right)} d_{j}^{2} \leq C \delta^{l-1 / 2} \beta_{j}^{2}
$$

for $\delta \geq 2 \theta^{-1} \delta_{j}$, so we also have (using again the strong convergence of $v_{j}\left(x+\psi_{j}(x)\right)$ in $\mathbf{C}^{(0)} \cap B_{\rho}$ for $\left.\rho<\frac{1}{4}\right)$ that

$$
\lim \int_{M_{j} \cap B_{\rho}} \beta_{j}^{-2} d_{j}^{2}=\int_{\mathbf{C}^{(0)} \cap B_{\rho}}|v|^{2}, \quad \rho<\frac{1}{4} .
$$

Now all of the above was computed relative to an arbitrary sequence $\mathbf{C}_{j} \in$ $\widetilde{\mathscr{C}}_{\varepsilon_{j}}\left(\mathbf{C}^{(0)}\right)$, and (since $\varepsilon_{j} \downarrow 0$ ) is equally valid if we choose a new cylinders $\widetilde{\mathbf{C}}_{j} \in \widetilde{\mathscr{C}}_{\gamma \varepsilon_{j}}\left(\mathbf{C}^{(0)}\right.$ ) (where $\gamma \geq 1$ is arbitrary) in place of $\mathbf{C}_{j}$. Of course in this case we get new quantities $\tilde{d}_{j}, \tilde{u}_{j}, \tilde{v}$ in place of $d_{j}, u_{j}, v$ respectively, but, for $j$ sufficiently large depending on $\gamma$, the estimates above remain equally valid, with constants $C$ only depending on $\mathbf{C}^{(0)}$, as before; notice particularly that the constants $C$ do not depend on $\gamma$. So let $\mathscr{L}$ be the linear subspace of solutions of $\mathscr{L}_{\mathrm{C}^{(0)}} w=0$ described in $\left(4.11^{\prime}\right)$. Because of the integrability condition $\ddagger \ddagger$ for the cross section $\mathbf{C}_{0}^{(0)}$, by (2.3) we know that, for each $\beta>0$ and for each $\psi \in \mathscr{L}$ with 
$\sup _{\mathbf{C}^{(0)} \cap B_{1}}|\psi| \leq \beta$, we can find $\widetilde{\mathbf{C}}_{j} \in \widetilde{\mathscr{C}}_{\gamma \beta_{j}}\left(\mathbf{C}^{(0)}\right)\left(\gamma=\gamma\left(\mathbf{C}^{(0)}, \beta\right) \geq 1\right)$ such that the new function $\tilde{u}_{j}$ (i.e., the function corresponding to $u_{j}$ when $\mathbf{C}_{j}$ is replaced by $\widetilde{\mathbf{C}}_{j}$ in the above discussion) satisfies a relation of the form

$$
\tilde{u}_{j}\left((x, y)+\tilde{\psi}_{j}(x, y)\right)=u_{j}\left((x, y)+\psi_{j}(x, y)\right)-\beta_{j} \psi+\widetilde{R}_{j}
$$

for $(x, y) \in \mathbf{C}^{(0)} \cap B_{3 / 4}$ with $|x| \geq \tilde{\tau}_{j}$, where $\tilde{\tau}_{j} \downarrow 0$, and $\beta_{j}^{-1}\left|\tilde{R}_{j}\right| \rightarrow 0$ as $j \rightarrow \infty$. Thus $\tilde{v}=v-\psi$ and in place of (10), (11), and (12) we obtain, for any constant $\beta>0$ and $\psi \in \mathscr{L}$ with $\sup |\psi| \leq \beta$,

$$
\rho^{l+3 / 2} \int_{\mathbf{C}^{(0)} \cap B_{\rho / 2}} \frac{\left|v-\psi-\kappa_{\psi, \rho}\right|^{2}}{r^{l+3 / 2}} \leq C \int_{\mathbf{C}^{(0)} \cap B_{\rho}}|v-\psi|^{2},
$$

where $\kappa_{\psi, \rho}(r \omega, y)=\sum_{i=1}^{l+k} \kappa_{\psi, \rho}^{i}(r, y) e_{i}^{\perp}$, with

$$
\sup _{\mathbf{C}^{(0)} \cap B_{\rho}} \sum_{i=1}^{l+k}\left(\kappa_{\psi, \rho}^{i}\right)^{2} \leq C \rho^{-n} \int_{\mathbf{C}^{(0)} \cap B_{\rho}}|v-\psi|^{2},
$$

and

$$
\int_{\mathbf{C}^{(0)} \cap B_{\rho / 2}} R^{2-n}\left(\frac{\partial(v / R)}{\partial R}\right)^{2} \leq C \rho^{-n-2} \int_{\mathbf{C}^{(0)} \cap B_{\rho}}|v-\psi|^{2},
$$

where $C=C\left(\mathbf{C}^{(0)}, \mathscr{M}\right)$, and the inequalities are valid for arbitrary $\rho \in$ $\left[\theta, \frac{1}{2}\right]$ and arbitrary $\beta>0$; we emphasize that the constant $C$ is independent of $\beta, \theta$. Notice that in (12) we used the fact that $\partial(v / R) / \partial R=$ $\partial((v-\psi) / R) / \partial R$, which is true because $\psi$ is homogeneous of degree one by definition of $\mathscr{L}$. Notice also that in place of (14) we obtain the identity

$$
\lim \int_{M_{j} \cap B_{\rho}} \beta_{j}^{-2} \tilde{d}_{j}^{2}=\int_{\mathbf{C}^{(0)} \cap B_{\rho}}|v-\psi|^{2}, \quad \rho<\frac{1}{4},
$$

where $\tilde{d}_{j}(X)=\operatorname{dist}\left(X, \widetilde{\mathbf{C}}_{j}\right), X \in M_{j}$.

Now (see (4.13)) for $\rho \in\left[\theta, \frac{1}{4}\right]$, we have by definition that $v_{\rho}=v-\psi_{\rho}$ with

$$
\int_{\mathbf{C}^{(0)} \cap B_{\rho}}\left|v-\psi_{\rho}\right|^{2}=\inf _{\psi \in \mathscr{L}} \int_{\mathbf{C}^{(0)} \cap B_{\rho}}|v-\psi|^{2},
$$

and hence (since $\int_{\mathbf{C}^{(0)} \cap B_{1}}|v|^{2} \leq 1$ ) we have

$$
\rho^{-n} \int_{\mathbf{C}^{(0)} \cap B_{\rho}}\left|\psi_{\rho}\right|^{2} \leq C \theta^{-2 n}, \quad C=C\left(\mathbf{C}^{(0)}\right) .
$$


Thus by elliptic estimates and homogeneity of $\psi$ we have $\sup _{\mathbf{C}^{(0)} \cap B_{1}}\left|\psi_{\rho}\right| \leq$ $C \theta^{-n}$ for $\rho \in\left[\theta, \frac{1}{4}\right]$, and we can apply the above with $\beta=C \theta^{-n}, \gamma=$ $\gamma\left(\mathbf{C}^{(0)}, \theta\right)$, and $\psi=\psi_{\rho}$ in order to conclude from $(10)^{\prime},(11)^{\prime}$, and $(12)^{\prime}$ that, at least for $l>2, v$ satisfies all the estimates needed to apply Lemma 4.14 (with $\alpha=\frac{1}{2}$ ). So we conclude that there is $\alpha=\alpha\left(\mathbf{C}^{(0)}, \mathscr{M}\right) \in(0,1)$ and $C=C\left(\mathbf{C}^{(0)}, \mathscr{M}\right)$ such that

$$
\theta^{-n-2} \int_{\mathbf{C}^{(0)} \cap B_{\theta}}\left|v_{\theta}\right|^{2} \leq C \theta^{\alpha} \int_{\mathbf{C}^{(0)} \cap B_{1}}\left|v_{1}\right|^{2} \leq C \theta^{\alpha},
$$

where we used the fact that $\int_{\mathbf{C}^{(0)} \cap B_{1}}\left|v_{1}\right|^{2} \leq \int_{\mathbf{C}^{(0)} \cap B_{1}}|v|^{2} \leq 1$ by construction. On the other hand by virtue of $(14)^{\prime}$ with $\rho=\theta$ (and with $\psi=\psi_{\theta}$ ), we have

$$
\lim _{j \rightarrow \infty} \int_{M_{j} \cap B_{\theta}} \beta_{j}^{-2} \tilde{d}_{j}^{2}=\int_{\mathbf{C}^{(0)} \cap B_{\theta}}\left|v_{\theta}\right|^{2},
$$

and hence (15) implies

$$
\theta^{-n-2} \int_{M_{j} \cap B_{\theta}} \tilde{d}_{j}^{2} \leq 2 \beta_{j}^{2} C \theta^{\alpha}
$$

for all sufficiently large $j$, which is (1) as required.

Notice that the same proof will apply also to the case $l=1$ if we can check the condition

$$
\lim _{r \downarrow 0} \frac{\partial^{2}}{\partial r \partial y^{i}} \sum_{\omega \in \Sigma_{0}} v(r \omega, y)=0, \quad|y|<\frac{1}{4},
$$

as required in order to apply Lemma 4.14. To check (16), we proceed as follows. Choose $\zeta=\zeta(r, y)$ to be smooth for $0 \leq r<\infty, y \in \mathbf{R}^{m}$ such that $\zeta(r, y) \equiv 0$ for $r^{2}+|y|^{2} \geq \frac{1}{4}$ and $\partial \zeta / \partial r=0$ in a neighborhood of $r=0$. Notice that then in particular we have

$$
D_{q} \zeta(|x|, y)=0 \text { for }|x|<\tau, q=1, \cdots, 1+k,
$$

for suitable $\tau>0$. For $i \in\{1, \cdots, 1+k\}, a \in\{1, \cdots, m\}$ we have by (1.1) that

$$
\int_{\widetilde{M}_{j}} \nabla x^{i} \cdot \nabla \zeta_{a}=\int_{\widetilde{M}_{j}} e_{i} \cdot \nabla \zeta_{a}=0, \quad \widetilde{M}_{j}=q_{j}^{-1} M_{j},
$$

where $\zeta_{a}=\partial \zeta / \partial y^{a}$. Let $g_{j}^{p q}$ denote the matrix of the orthogonal projection of $\mathbf{R}^{n+k}$ onto $T_{(x, y)} \widetilde{M}_{j}$, let $G_{j}=\operatorname{graph}\left(u_{j} \mid U_{j} \cap B_{1 / 2}\right)$, with $U_{j}, u_{j}$ 
corresponding to $U, u$ as in 2.6 with $\widetilde{M}_{j}$ in place of $M$, and note that, by Lemma $2.6, \widetilde{M}_{j} \cap B_{1 / 2} \backslash G_{j} \subset\{(x, y):|x|<\tau\}$ for all sufficiently large $j$. Since

$$
\int_{\widetilde{M}_{j} \backslash G_{j}}\left|\nabla x^{i} \cdot \nabla \zeta_{a}\right|=\int_{\widetilde{M}_{j} \backslash G_{j}}\left|\sum_{p=1}^{n+k} g_{j}^{i p}(X) D_{p} \zeta_{a}\right|,
$$

and $D_{p} \zeta_{a}=0 \forall p=1, \cdots, 1+k,|x|<\tau$, we have for $|x|<\tau$ that

$$
\begin{aligned}
\left|\sum_{p=1}^{n+k} g^{i p} D_{p} \zeta_{a}\right| & =\left|\sum_{p=1}^{n-1}\left(\delta_{i, 1+k+p}-g^{i, 1+k+p}\right) D_{y^{p}} \zeta_{a}\right| \\
& \leq \sqrt{\sum_{p=1}^{n-1}\left|e_{1+k+p}^{\perp}\right|^{2}\left|D \zeta_{a}\right| .}
\end{aligned}
$$

So, for $j$ large enough, (19) together with (1.5) and Theorem 3.1 gives

$$
\begin{aligned}
& \int_{\widetilde{M}_{j} \backslash G_{j}}\left|\nabla x^{i} \cdot \nabla \zeta_{a}\right| \\
& \leq \sqrt{\mathscr{H}^{n}\left(\widetilde{M}_{j} \cap B_{1 / 2} \backslash G_{j}\right)} \sqrt{\int_{\widetilde{M}_{j}} \sum_{p=1}^{n-1}\left|e_{1+k+p}^{\perp}\right|^{2}} \sup \left|D \zeta_{a}\right| \\
& \quad \leq C \sqrt{\tau} \sqrt{\left(\int_{\widetilde{M}_{j}} \sum_{p=1}^{n-1}\left|e_{1+k+p}^{\perp}\right|^{2}\right)^{1 / 2}} \sup \left|D \zeta_{a}\right| .
\end{aligned}
$$

Now let $\omega_{j}^{(1)}, \cdots, \omega_{j}^{(N)}$ be unit vectors in the direction of the singular rays of the $j$-dimensional cross section $\mathbf{C}_{0}^{(j)}$ of the cylinder $\mathbf{C}_{0}^{(j)} \times \mathbf{R}^{n-1} \equiv$ $q_{j}^{-1} \mathbf{C}_{j}$, and write

$$
U_{j}(\tau)=U_{j} \cap\left\{(x, y) \in \mathbf{R}^{1+k} \times \mathbf{R}^{m}:|x|>\tau\right\}=\bigcup_{i=1}^{N} U_{j}^{(i)}(\tau),
$$

where

$$
U_{j}^{(i)}(\tau)=\left\{\left(x, \omega_{j}^{(i)}, y\right): y \in \mathbf{R}^{n-1}, x>\tau\right\}
$$

Also, define

$$
G_{j}(\tau)=\operatorname{graph}\left(u_{j} \mid U_{j}(\tau)\right), \quad G_{j}^{(i)}(\tau)=\operatorname{graph}\left(u_{j} \mid U_{j}^{(i)}(\tau)\right) .
$$

Next note that $\left|e_{1+k+p}^{\perp}\right| \geq \frac{1}{2}\left|D_{y^{p}} u_{j}\right|$ on $U_{j}(\tau)$ for $j$ sufficiently large (depending on $\tau$ ), and hence the first estimate of Theorem 3.1 implies that

$$
D_{y^{a}} v \in L^{2}\left(\mathbf{C}^{(0)} \cap B_{1 / 2}\right) \text {. }
$$


Suppose for the moment that $\omega_{j}^{(1)}=e_{1}$. Then

$$
\begin{aligned}
& \int_{G_{j}^{(1)}(\tau)} \nabla x^{1} \cdot \nabla \zeta_{a} \\
&=\int_{0}^{1} \int_{\mathbf{R}^{m}} \sqrt{h_{j}}\left\{h_{j}^{11}(x, y) \frac{\partial}{\partial x}\left(\zeta_{a}\left(\sqrt{x^{2}+\left|u_{j}\right|^{2}}, y\right)\right)\right. \\
&\left.\quad+\sum_{p=1}^{n-1} h_{j}^{1,1+k+p} \frac{\partial}{\partial y^{p}}\left(\zeta_{a}\left(\sqrt{x^{2}+\left|u_{j}\right|^{2}}, y\right)\right)\right\} d y d x
\end{aligned}
$$

where $\left(h^{p q}\right)_{p, q \notin\{2, \cdots, 1+k\}}$ is the inverse of the $n \times n$ matrix $\left(\delta_{p q}+D_{p} u \cdot D_{q} u\right)_{p, q \notin\{2, \cdots, 1+k\}}$, and $h_{j}$ is the determinant of this matrix. Now

$$
\begin{aligned}
\zeta_{a}\left(\sqrt{x^{2}+|u|^{2}}, y\right)= & \frac{\partial}{\partial y^{a}}\left(\zeta\left(\sqrt{x^{2}+\left|u_{j}\right|^{2}}, y\right)\right) \\
& -\frac{(\partial \zeta / \partial r)\left(\sqrt{\left.x^{2}+\left|u_{j}\right|^{2}, y\right)} u_{j} \cdot \partial u_{j} / \partial y^{a}\right.}{\sqrt{x^{2}+\left|u_{j}\right|^{2}}}
\end{aligned}
$$

and hence after an integration with respect to $y^{a}$, we can write

$$
\begin{aligned}
\int_{0}^{1} \int_{\mathbf{R}^{m}} & \sqrt{h_{j}}\left\{h_{j}^{11}(x, y) \frac{\partial}{\partial x}\left(\zeta_{a}\left(\sqrt{x^{2}+\left|u_{j}\right|^{2}}, y\right)\right)\right\} \\
= & \int_{0}^{1} \int_{\mathbf{R}^{n-1}}\left\{\left(\sqrt{h_{j}} h_{j}^{11}-1\right) \frac{\partial^{2} \zeta}{\partial y^{a} \partial x}\left(\sqrt{x^{2}+\left|u_{j}\right|^{2}}, y\right)\right. \\
& \left.-\sqrt{h_{j}} h_{j}^{11} \frac{\partial}{\partial x}\left(\frac{(\partial \zeta / \partial r)\left(\sqrt{x^{2}+\left|u_{j}\right|^{2}, y}\right) u_{j} \cdot \partial u_{j} / \partial y^{a}}{\sqrt{x^{2}+\left|u_{j}\right|^{2}}}\right)\right\} .
\end{aligned}
$$

Also

$$
\left|h^{1,1+k+p}(x, y)\right| \leq C\left|\nabla u_{j}\right|^{2}, \quad\left|\sqrt{h_{j}} h_{j}^{11}-1\right| \leq C\left|\nabla u_{j}\right|^{2} \text { on } U_{j}^{(1)},
$$

as one checks from the fact that $\left(h_{j}^{p q}\right)$ is the inverse of $\left(\delta_{p q}+D_{p} u_{j} \cdot D_{q} u_{j}\right)_{p, q \notin\{2, \cdots, 1=k\}}$, and hence using (23) and (24) in (22) by standard elliptic estimates we get, for $j$ sufficiently large (depending on $\tau$ ), 


$$
\begin{aligned}
& \left|\int_{G_{j}^{(1)}(\tau)} \nabla x^{1} \cdot \nabla \zeta_{a}\right| \\
& \quad \leq C \int_{U_{j}^{(1)}(3 \tau / 4)}\left(\left|\nabla u_{j}\right|^{2}+\left|u_{j}\right|\left(\left|\nabla^{2} u_{j}\right|+\left|\nabla u_{j}\right|\right)\right)\left(|\nabla \zeta|+\nabla^{2} \zeta \mid\right) \\
& \quad \leq C\left(\int_{U_{j}^{(1)}(\tau / 2) \cap B_{1}} u_{j}^{2}\right) \sup \left(|\nabla \zeta|+\left|\nabla^{2} \zeta\right|\right)
\end{aligned}
$$

where $C$ depends on $\tau$ but not on $j$. Also, since $G_{j}^{(1)}$ is given by $x^{i}=$ $u^{i}(x, 0, y), i=2, \cdots, 1+k$, for $i \geq 2$ we have

$$
\begin{aligned}
\int_{G_{j}^{(1)}(\tau)} & \nabla x^{i} \cdot \nabla \zeta_{a} \\
= & \int_{U_{j}^{(1)}(\tau)} \sum_{p, q \neq 2, \cdots, 1+k} h_{j}^{p q}\left(D_{p} u_{j}^{i}\right)\left(D_{q}\left(\zeta_{a}\left(\sqrt{x^{2}+|u|^{2}}, y\right)\right)\right) \sqrt{h_{j}} \\
= & \int_{U_{j}^{(1)}(\tau)}\left(\nabla u_{j}^{i} \cdot \nabla \zeta_{a}+R_{j}\right),
\end{aligned}
$$

where $\left|R_{j}\right| \leq C\left|\nabla u_{j}\right|^{2} \sup \left(\left|D \zeta_{a}\right|+\mid D^{2}\left(\zeta_{a} \mid\right)\right.$, and $\zeta_{a} \nabla$ is the gradient operator on $q_{j}^{-1} \mathbf{C}_{j}$. Combining (25) and (26) yields

$$
\int_{G_{j}^{(1)}(\tau)} \sum_{i=1}^{1+k}\left(e_{i} \cdot \nabla \zeta_{a}\right) e_{i}=\int_{U_{j}^{(1)}(\tau)} \nabla u \cdot \nabla \zeta_{a}+R_{j},
$$

where $\beta_{j}^{-1} R_{j} \rightarrow 0$ as $j \rightarrow \infty$. This is an invariant expression relative to orthogonal transformations of $\mathbf{R}^{1+k}$, and hence it holds without the assumption $\omega_{j}^{(1)}=e_{1}$, and a similar formula holds for each $G_{j}^{(i)}(\tau)$. Thus

$$
\int_{G_{j}(\tau)} \sum_{i=1}^{1+k}\left(e_{i} \cdot \nabla \zeta_{a}\right) e_{i}=\int_{U_{j}(\tau)} \nabla u \cdot \nabla \zeta_{a}+R_{j},
$$

with $\beta_{j}^{-1} R_{j} \rightarrow 0$ as $j \rightarrow \infty$. Combining this with (20) and using (18) we conclude that

$$
0=\int_{\widetilde{M}_{j}} \sum_{i=1}^{1+k}\left(e_{i} \cdot \nabla \zeta_{a}\right) e_{i}=\int_{U_{j}} \nabla u_{j} \cdot \nabla \zeta_{a}+R_{j}+S_{j} \beta_{j},
$$

where $\beta_{j}^{-1} R_{j} \rightarrow 0$ as $j \rightarrow \infty$, and $\left|S_{j}\right| \leq C \sqrt{\tau}$. Since $v \in L^{2}\left(\mathbf{C}^{(0)} \cap B_{1 / 2}\right)$ and $D_{y} v \in L^{2}\left(\mathbf{C}^{(0)} \cap B_{1 / 2}\right)$ by (21), we can multiply by $\beta_{j}^{-1}$ and first take limits in $j$ and then let $\tau \downarrow 0$, thus giving 


$$
\int_{\mathbf{C}^{(0)}} \nabla v \cdot \nabla \zeta_{a}=0
$$

whenever $\partial \zeta / \partial r=0$ in some neighborhood of $r=0$ and $\zeta \in C_{c}^{\infty}\left(B_{1 / 4}^{n}\right)$.

Since $\zeta$ is a function of $r, y$ only, after an integration by parts, the above equation can be written $\int_{H} \tilde{v} \quad \Delta \zeta_{a}=0$, where $H$ is the half-space $\left\{(r, y) \in \mathbf{R}^{n}: r>0\right\}$, and $\tilde{v}(r, y)=\sum_{j=1}^{N} v\left(r \omega_{j}, y\right)$. In view of the arbitrariness of $\zeta$, this evidently implies

$$
\int_{H} \tilde{v} \cdot \delta_{a, h} \Delta \zeta=0, \quad|h|<\frac{1}{4},
$$

provided $\zeta$ is smooth with $\partial \zeta / \partial r=0$ in a neighborhood of $r=0$ and $\zeta \equiv 0$ for $\sqrt{r^{2}+|y|^{2}} \geq \frac{1}{4}$, where $\delta_{a, h} \zeta(r, y)=\zeta\left(r, y+h e_{l+k+a}\right)-\zeta(r, y)$. This in turn clearly yields that

$$
\int_{H}\left(\delta_{a, h} \tilde{v}\right) \cdot \Delta \zeta=0, \quad|h|<\frac{1}{4} .
$$

Now for any function $\zeta \in C_{c}^{\infty}\left(B_{1 / 4}^{n}\right)$ with $\zeta(r, y) \equiv \zeta(-r, y)$ we can find a sequence $\zeta_{k}$ such that the derivatives of $\zeta_{k}$ up to and including order 2 are bounded independent of $k, \zeta_{k} \rightarrow \zeta$ in the $C^{2}$ norm locally in $B_{1 / 4}^{n} \backslash\{0\} \times \mathbf{R}^{n-1}$, and $\partial \zeta_{k} / \partial r \equiv 0$ for $|r|<\tau_{k}$ with $\tau_{k}>0$ and $\tau_{k} \downarrow 0$. Thus using the dominated convergence theorem we in fact deduce that (27) holds for any $\zeta \in C_{c}^{\infty}\left(B_{1 / 4}^{n}\right)$ with $\zeta(-r, y)=\zeta(r, y)$. Of course then

$$
\int_{B_{1 / 4}^{n}}\left(\delta_{a, h} \hat{v}\right) \cdot \Delta \zeta=0, \quad|h|<\frac{1}{4},
$$

where $\hat{v}$ is the even extension $\hat{v}(r, y)=\hat{v}(-r, y)$. On the other hand we trivially have (28) if $\zeta(r, y)=-\zeta(-r, y)$ (even if $\zeta$ does not have compact support). Since any $\zeta \in C_{c}^{\infty}\left(B_{1 / 4}^{n}\right)$ can be written as the sum $\frac{1}{2}(\zeta(r, y)+\zeta(-r, y))+\frac{1}{2}(\zeta(r, y)-\zeta(-r, y))$, we conclude that in fact (28) holds for any $\zeta \in C_{c}^{\infty}\left(B_{1 / 4}\right)$, and Weyl's lemma tells us that $\delta_{a, h} \hat{v}$ is a smooth harmonic function on $B_{1 / 4}$ for $|h|<\frac{1}{4}$.

Since by (21) $h^{-1} \delta_{a, h} \hat{v} \rightarrow D_{y^{a}} \hat{v}$ in $L^{2}\left(B_{1 / 4}^{n}\right)$ as $h \rightarrow 0$, it then follows that $D_{y^{a}} v$ also extends to give a harmonic function which is even in the $r$-variable. In particular we have (16) as required. Hence the proof is complete.

5.2 Proof of Theorem 1. Choose $\theta \in\left(0, \frac{1}{8}\right)$ such that

$$
C \theta^{\alpha} \leq 1 / 4 \text {, }
$$


where $C$ and $\alpha$ are as in Lemma 1. Let $\operatorname{sing}_{*} M=\left\{X \in B_{1 / 2}: \Theta_{M}(X) \geq\right.$ $\left.\boldsymbol{\Theta}_{\mathbf{C}^{(0)}}(0)\right\}$, and let $\varepsilon_{0}, \delta_{0}$ (depending only on $\mathbf{C}^{(0)}, \mathscr{M}$ ) be small enough to ensure the conclusions of Lemma 1 with the above choice of $\theta$. We also assume $\varepsilon_{0}$ is less than the $\varepsilon_{0}$ of Theorem 3.1 when $\alpha$ and $\gamma$ of Theorem 3.1 are both equal to $\frac{1}{2}$ and $\tau=10^{-2}$. Let $M$ satisfy the hypotheses of Theorem 1 with $C \varepsilon \leq \varepsilon_{0}$, where $C=C\left(\mathbf{C}^{(0)}, \mathscr{M}\right) \geq 1$ is to be chosen $(\geq$ the choice of $\gamma$ of Lemma 1), and define $E_{j}, j=0,1, \cdots$, as follows. $E_{0}$ is the set of $X \in \operatorname{sing}_{*} M$ such that $\eta_{X, \rho} M$ satisfies alternative (i) of Lemma 1 for some $\rho \in(\theta, 1]$, and, for $j \geq 1$, let $E_{j}$ be the set of $X \in \operatorname{sing}_{*} M$ such that $\eta_{X, \rho} M$ does not satisfy alternative (i) of Lemma 1 (hence does satisfy (**) of Remark 1.14) if $\rho=\theta^{i}, i=0, \cdots, j$, and such that $\eta_{X, \rho} M$ does satisfy the alternative (i) of Lemma 1 when $\rho=\theta^{j+1}$. Finally, $E_{\infty}$ is the set of $X \in \operatorname{sing}_{*} M$ such that $\eta_{X, \rho} M$ does not satisfy alternative (i) of Lemma 1 (and hence does satisfy (**) of Remark 1.14) for all $\rho=\theta^{i}, i=0,1,2, \cdots$.

First note that if $E_{0} \neq \varnothing$ then, keeping in mind (1.5) and the fact that, by Lemma 2.6, sing $M \cap B_{1} \subset$ the $\delta(\varepsilon)$-neighborhood of $\{0\} \times \mathbf{R}^{m}$, with $\delta(\varepsilon) \downarrow 0$ as $\varepsilon \downarrow 0$, we trivially have the conclusion of Theorem 1 with $S=\varnothing$ and $T=\operatorname{sing} M \cap B_{1}$. Thus from now on we assume

$$
E_{0}=\varnothing \text {. }
$$

Next we want to show that $E_{\infty} \subset L$, with $L$ as in the statement of the theorem. We are going to show that the theorem holds with $S=E_{\infty}$ and $T=\left(\operatorname{sing}_{*} M \backslash E_{\infty}\right) \cup\left(\operatorname{sing} M \cap B_{1} \backslash B_{1 / 2}\right)$.

Take $X_{0} \in E_{\infty}$. Using the definition of $E_{\infty}$, we see that, provided $\gamma \varepsilon \leq \varepsilon_{0}$ with $\gamma=\gamma\left(\mathbf{C}^{(0)}, \mathscr{M}\right)$ is sufficiently large, we can iterate Lemma 1 , inductively choosing $\mathbf{C}_{0}, \mathbf{C}_{1}, \cdots$, with $\mathbf{C}_{0}=\mathbf{C}^{(0)}, \mathbf{C}_{i} \in \tilde{\mathscr{C}}_{\gamma \varepsilon_{0}}\left(\mathbf{C}^{(0)}\right)$, and $q_{i}=\exp A_{i}$, with $A_{i} \in \mathscr{S}\left(\mathscr{S}\right.$ as in Definition 1.12) $\left|A_{i}\right| \leq \gamma \varepsilon_{0}$ and $q_{i}^{-1} \mathbf{C}_{i} \in \mathscr{C}_{\gamma \varepsilon_{0}}\left(\mathbf{C}^{(0)}\right)$; as the inductive step we apply Lemma 1 with $q_{i}^{-1} \eta_{X_{0}, \theta^{i}} M$ in place of $M$, giving $\mathbf{C}_{i+1} \in \widetilde{\mathscr{C}}_{\gamma \varepsilon_{0}}\left(\mathbf{C}^{(0)}\right)$, such that

$$
\begin{gathered}
\theta^{-(i+1)(n+2)} \int_{M_{0} \cap B_{\theta} i+1} \operatorname{dist}^{2}\left(X, \mathbf{C}_{i+1}\right) \leq \frac{1}{4} \theta^{-i(n+2)} \int_{M_{0} \cap B_{\theta} i} \operatorname{dist}^{2}\left(X, \mathbf{C}_{i}\right) \\
\leq 2^{-2 i} \int_{M_{0} \cap B_{1}} \operatorname{dist}^{2}\left(X, \mathbf{C}^{(0)}\right) \leq 2^{-2 i} \varepsilon^{2},
\end{gathered}
$$

where $M_{0}=\tau_{X_{0}} M\left(\tau_{X_{0}}\right.$ the translation $\left.X \mapsto X-X_{0}\right)$, and

$$
\text { Hausdorff distance }\left(\mathbf{C}_{i} \cap B_{1}, \mathbf{C}_{i+1} \cap B_{1}\right) \leq \gamma \varepsilon 2^{-i},
$$


subject to the inductive hypothesis that this holds with $j \leq i-1$ in place of $i$; notice that we need to check at each stage that $q_{i}^{-1} \eta_{X_{0}, \theta^{i}} M \in \mathscr{N}_{\varepsilon_{0}}\left(\mathbf{C}^{(0)}\right)$, but this is an easy consequence of Lemma 1 since $C \varepsilon \leq \varepsilon_{0}$. Notice also that (3) actually implies (4) for suitable $\gamma=\gamma\left(\mathbf{C}^{(0)}, \mathscr{M}, \theta\right)$ by virtue of the remark following Lemma 2.6.

In particular (4) implies that there exists $\mathbf{C}_{X_{0}} \in \widetilde{\mathscr{C}}_{\gamma \varepsilon}\left(\mathbf{C}^{(0)}\right)$ such that

$$
\text { Hausdorff distance }\left(\mathbf{C}_{i} \cap B_{1}, \mathbf{C}_{X_{0}} \cap B_{1}\right) \leq 2 \gamma \varepsilon 2^{-i}
$$

and

$$
\theta^{-i(n+2)} \int_{M_{0} \cap B_{\theta} i} \operatorname{dist}^{2}\left(X, \mathbf{C}_{X_{0}}\right) \leq \frac{C}{2^{i}} \int_{M_{0} \cap B_{1}} \operatorname{dist}^{2}\left(X, \mathbf{C}^{(0)}\right),
$$

for each $i=1,2, \cdots$. Thus

$$
\rho^{-n-2} \int_{M_{0} \cap B_{\rho}} \operatorname{dist}^{2}\left(X, \mathbf{C}_{X_{0}}\right) \leq C \rho^{2 \mu} \int_{M_{0} \cap B_{1}} \operatorname{dist}^{2}\left(X, \mathbf{C}^{(0)}\right)
$$

for all $\rho \in(0,1]$, with $\mu=\mu(\theta)$ chosen so that $\theta^{2 \mu}=\frac{1}{2}$.

By definition there is $q_{X_{0}} \in \mathrm{SO}\left(\mathbf{R}^{n+k}\right)$ such that $q_{X_{0}} \mathbf{C}_{X_{0}} \in \mathscr{C}_{\gamma \varepsilon}\left(C^{(0)}\right)$ and

$$
\left|q_{X_{0}}-\mathbf{1}\right| \leq C \varepsilon
$$

and by applying the first estimate in Theorem 3.1 (with $q_{X_{0}} \eta_{X_{0}, \rho} M$ in place of $M)$ we have, for $\rho \in(0,1]$,

$$
\rho^{-1-\mu} \operatorname{dist}\left(q_{X_{0}} \tau_{X_{0}}\left(\operatorname{sing}_{*} M\right) \cap B_{\rho},\{0\} \times \mathbf{R}^{m}\right) \leq C \varepsilon,
$$

where $\tau_{X_{0}}$ is the translation $X \mapsto X-X_{0}$. Also, again by a standard argument based on (3), (4),

$$
\left|q_{X_{0}}-q_{Y_{0}}\right| \leq C \varepsilon\left|X_{0}-Y_{0}\right|^{\mu} .
$$

Since this is valid (with fixed constant $C=C\left(\mathbf{C}^{(0)}, \mathscr{M}\right)$ ) for any $X_{0}, Y_{0} \in$ $E_{\infty}$, we can then write

$$
E_{\infty} \subset \operatorname{graph} v \equiv\left\{(x, v(x)): x \in\left[-\frac{1}{2}, \frac{1}{2}\right]^{m}\right\},
$$

where $v:\left[-\frac{1}{2}, \frac{1}{2}\right]^{m} \rightarrow \mathbf{R}^{l+k}$ satisfies

$$
|v|_{C^{1, \mu}} \leq C \varepsilon, \quad C=C\left(\mathbf{C}^{(0)}\right) .
$$

So $L=\operatorname{graph} v$ has the properties stated in Theorem 1, assuming we take $S=E_{\infty}$. Now in view of (2) and the definition of $E_{j}$, we have

$$
\operatorname{sing}_{*} M \backslash \operatorname{graph} v=\bigcup_{j=1}^{\infty} E_{j} .
$$


Also, we note that for points $X_{0} \in E_{j}$ we can apply precisely the same iterative argument (based on repeated use of Lemma 1) as we used for $E_{\infty}$, except that now we conclude (3), (4) only for $i=1, \cdots, j$, and correspondingly (6) is only valid for $\rho \geq \theta^{j}$. That is, we can still select fixed $q_{X_{0}}$ and $C_{X_{0}}$ (no longer unique) such that (5) holds and

$$
\rho^{-1-\mu} \operatorname{dist}\left(q_{X_{0}} \tau_{X_{0}}\left(\operatorname{sing}_{*} M\right) \cap B_{\rho},\{0\} \times \mathbf{R}^{m}\right) \leq C \varepsilon, \quad \theta^{j} \leq \rho \leq 1 .
$$

Notice that in particular, in view of (5) this gives

$$
\begin{gathered}
\rho^{-1} \operatorname{dist}\left(\operatorname{sing}_{*} M \cap B_{\rho}\left(X_{0}\right), X_{0}+\left(\{0\} \times \mathbf{R}^{m}\right)\right) \leq C \varepsilon, \\
\theta^{j} \leq \rho \leq 1, X_{0} \in E_{j} .
\end{gathered}
$$

Also by definition of $E_{j}$ we have the additional fact that

(13) $\forall X_{0} \in E_{j}$,

$$
\exists Y \in X_{0}+\left(\{0\} \times B_{\theta^{j}}^{m}(0)\right) \quad \text { with } B_{\delta_{0} \theta^{j}}(Y) \cap \operatorname{sing}_{*} M=\varnothing,
$$

where $\delta_{0}=\delta_{0}\left(\mathbf{C}^{(0)}, \mathscr{M}\right)$ is as in Lemma 1 . By combining (12) and (13) it is now straightforward to check that $E_{j}$ can be covered by a collection $\mathscr{R}_{j}$ of cubes $R=(\xi, \eta)+\left[-\theta^{j} / 2, \theta^{j} / 2\right]^{n+k}$, where $(\xi, \eta) \in E_{j}$,

$$
\begin{aligned}
& \left(\mathbf{R}^{l+k} \times\left(\eta+\left[-\theta^{j} / 2, \theta^{j} / 2\right]^{m}\right)\right) \cap \operatorname{sing}_{*} M \\
& \quad \subset(\xi, \eta)+\left(\left[-C \varepsilon \theta^{j}, C \varepsilon \theta^{j}\right]^{l+k} \times\left[-\theta^{j} / 2, \theta^{j} / 2\right]^{m}\right),
\end{aligned}
$$

and $R$ has a subcube $\left(\xi^{\prime}, \eta^{\prime}\right)+\left[-2 \delta \theta^{j}, 2 \delta \theta^{j}\right]^{n+k}$ such that

$$
\left(\mathbf{R}^{l+k} \times\left(\eta^{\prime}+\left[-2 \delta \theta^{j}, 2 \delta \theta^{j}\right]^{m}\right)\right) \cap \operatorname{sing}_{*} M=\varnothing,
$$

with $\delta=2^{-N}, N=N\left(\mathbf{C}^{(0)}, \mathscr{M}\right) \geq 4$.

Thus we can apply Lemma 2.7 to the collection $\mathscr{Q}$ of cubes in $\mathbf{R}^{m}$ which are obtained by orthogonal projections $\Pi_{\mathbf{R}^{m}} R$ onto $\{0\} \times \mathbf{R}^{m}$ of the cubes $R$ in the collection $\bigcup_{j=1}^{\infty} \mathscr{R}_{j}$ and with $F$ the orthogonal projection onto $\{0\} \times \mathbf{R}^{m}$ of $\bigcup_{j=1}^{\infty} E_{j}$. Then Lemma 2.7 gives a collection $\mathscr{P}$ of cubes in $\{0\} \times \mathbf{R}^{m}$ with

$$
\sum_{P \in \mathscr{P}} e(P)^{m} \leq 1-\delta^{2 m}, \quad F \subset \bigcup_{P \in \mathscr{P}} P,
$$

and with the property that for each $P \in \mathscr{P}$ there is a $Q \in \mathscr{Q}$ with

$$
P \subset Q^{(5)} \text { and }(\delta / 4) e(Q) \leq e(P) \leq e(Q) .
$$

Now for each such $P=\tilde{\eta}+[-\rho, \rho]^{m} \in \mathscr{P}$ and corresponding $Q=\Pi_{\mathbf{R}^{m}} R$ as in (17), where $R \in \bigcup_{j=1}^{\infty} \mathscr{R}_{j}$ has center $(\xi, \eta) \in \bigcup_{j \geq 1} E_{j}$, select an 
$(n+k)$-cube $\widetilde{R}$ with $\Pi_{\mathbf{R}^{m}} \widetilde{R}=P$ and with center $(\tilde{\xi}, \tilde{\eta})$ such that $\tilde{\xi}=\xi$. Let $\widetilde{R}$ be the collection of such cubes $\widetilde{R}$. Then by (12), (15), (16), and (17) we have for $\varepsilon$ sufficiently small depending on $\mathscr{M}, \mathbf{C}^{(0)}$,

$$
\bigcup_{j=1}^{\infty} E_{j} \subset \bigcup_{R \in \widetilde{\mathscr{R}}} R, \quad \sum_{R \in \widetilde{\mathscr{R}}} e(R)^{m} \leq 1-\delta^{2 m},
$$

and, by (14), for each $R \in \widetilde{\mathscr{R}}$ with center $(\xi, \eta) \in \mathbf{R}^{l+k} \times \mathbf{R}^{m}$,

$$
\bigcup_{j=1}^{\infty} E_{j} \cap R \subset\left(\xi+[-C \varepsilon e(R), C \varepsilon e(R)]^{l+k}\right) \times \mathbf{R}^{m}, \quad C=C\left(\mathbf{C}^{(0)}, \mathscr{M}\right) .
$$

Now the rest of Theorem 1 follows from the fact that for each $\alpha>0$ we can find balls $\left\{B_{\rho_{j}}\left(X_{j}\right)\right\}$ such that $X_{j} \in\{0\} \times\left[-\frac{1}{2}, \frac{1}{2}\right]^{m}$ and

$$
\left(-\varepsilon_{1}, \varepsilon_{1}\right)^{l+k} \times\left[-\frac{1}{2}, \frac{1}{2}\right]^{m} \subset \bigcup_{j=1}^{N} B_{\rho_{j}}\left(X_{j}\right), \quad \sum_{j=1}^{N} \omega_{m} \rho_{j}^{m} \leq 1+\alpha,
$$

where $\varepsilon_{1}=\varepsilon_{1}(\alpha, n, k) \in\left(0, \frac{1}{2}\right)$. Applying this to appropriate scalings and translations of $\{0\} \times\left[-\frac{1}{2}, \frac{1}{2}\right]^{m}$, and using (18) and (19), it then follows that we can replace our covering $\widetilde{\mathscr{R}}$ by a covering by balls with the required properties. Hence the proof of Theorem 1 is complete.

\section{Proofs of Theorems $2^{\prime}$ and 4 , and Corollary 3}

6.1. Proof of Theorem $2^{\prime}$. Let $m$ be as in (1.15), and let $\operatorname{sing}_{*} M$ denote the set of $X \in \operatorname{sing} M$ such that there exists a tangent cone $\mathbf{C} \in$ $\operatorname{Tan}_{X} M$ with $\mathbf{C}=q\left(\mathbf{C}_{0} \times \mathbf{R}^{m}\right)$ for some $q \in \mathrm{SO}\left(\mathbf{R}^{n+k}\right)$. Notice that by (1.10) we have

$$
\operatorname{dim}\left(\operatorname{sing} M \backslash \operatorname{sing}_{*} M\right) \leq m-1 .
$$

Let

$$
S_{\alpha}=\operatorname{sing}_{\alpha} M\left(\equiv \operatorname{sing}_{*} M \cap\left\{X: \Theta_{M}(X)=\alpha\right\}\right),
$$

$X_{0} \in S_{\alpha}, \varepsilon>0$, and let $\mathbf{C}^{(0)}=q\left(\mathbf{C}_{0}^{(0)} \times \mathbf{R}^{m}\right) \in \operatorname{Tan}_{X_{0}} M$. By the definition of $\operatorname{Tan}_{X_{0}} M$ and the compactness 1.3(b), for each $\varepsilon>0$ we can find $\sigma=\sigma(\varepsilon)>0$ such that $B_{\sigma}\left(X_{0}\right) \subset U_{M}$ and such that $M_{0}=\eta_{X_{0}, \sigma} M$ satisfies $U_{M_{0}} \supset B_{R(\varepsilon)}$ and

$$
\frac{\mathscr{H}^{n}\left(M_{0} \cap B_{R(\varepsilon)}\right)}{\omega_{n} R(\varepsilon)^{n}}-\Theta_{\mathbf{C}^{(0)}}(0)<\delta(\varepsilon), \quad \int_{M_{0} \cap B_{1}(0)} \operatorname{dist}^{2}(X, \mathbf{C})<\varepsilon^{2},
$$


where $\delta(\varepsilon)$ and $R(\varepsilon)$ are as in Lemma 2.4. Let

$$
S_{\alpha}^{+}=\left\{X \in \operatorname{sing} M_{0}: \Theta_{M_{0}}(X) \geq \alpha\right\} \quad\left(\supset\left(\eta_{X_{0}, \sigma} S_{\alpha}\right) \cap B_{1}\right) .
$$

Notice that $S_{\alpha}^{+}$is closed in $U_{M_{0}}$ by upper semicontinuity of $\Theta_{M_{0}}(X)$ (which is true by $(1.5)$ ). For given $\rho_{0} \in\left(0, \frac{1}{2}\right)$ take a finite cover of $S_{\alpha}^{+} \cap \bar{B}_{1}$ by balls $B_{\sigma_{i}}\left(Y_{i}\right)$ with $\sigma_{i} \leq \rho_{0}$ such that

$$
\sum_{i} \omega_{m} \sigma_{i}^{m} \leq \mu_{\rho_{0}}\left(S_{\alpha}^{+} \cap \bar{B}_{1}\right)+1,
$$

where $\mu_{\rho_{0}}$ is the outer measure defined by

$$
\mu_{\rho_{0}}(A)=\inf \sum_{j} \omega_{m} \rho_{j}^{m}
$$

taken over all countable collections $\left\{B_{\rho_{j}}\left(X_{j}\right)\right\}$ of balls with $A \subset \bigcup_{j} B_{\rho_{j}}\left(X_{j}\right)$ and $\rho_{j} \leq \rho_{0} \forall j$. Of course we may assume $S_{\alpha}^{+} \cap B_{\sigma_{i}}\left(Y_{i}\right) \neq \varnothing$ for each $i$, otherwise we can drop the $B_{\sigma_{i}}\left(Y_{i}\right)$ which do not satisfy this. For each $i$ choose $Z_{i} \in S_{\alpha}^{+} \cap B_{\sigma_{i}}\left(Y_{i}\right)$ and apply Lemma 2.4 to $M_{0}$. Then, for suitably small $\varepsilon>0$ (depending only on $\mathscr{M}, K$ ), either there exists a cylindrical cone $\mathbf{C}=q\left(\mathbf{C}_{0} \times \mathbf{R}^{m}\right) \in \mathscr{M}$ with $\boldsymbol{\Theta}_{C}(0)=\alpha$ and

$$
\sigma_{i}^{-2-n} \int_{\eta_{Z_{i}, 2 \sigma_{i}} M_{0} \cap B_{1}} \operatorname{dist}^{2}(X, \mathbf{C}) \leq \varepsilon^{2}
$$

or else

$$
\left\{X \in B_{2 \sigma_{i}}\left(Z_{i}\right): \Theta_{M_{0}}(X) \geq \alpha\right\} \subset\{X: \operatorname{dist}(X, H)<\varepsilon\}
$$

for some $(m-1)$-dimensional affine space $H$ containing $Z_{i}$. Notice that here we use the fact that by (2.1) and (2) we can choose $\varepsilon$ (depending only on $\mathscr{M}, K)$ to ensure that $\Theta_{\mathbf{C}}(0)=\alpha$ in (4); we henceforth assume that $\varepsilon>0$ is so chosen.

In the case of (4) we consider two subcases, namely, 4(i): $\exists \widetilde{Z}_{i} \in S_{\alpha}^{+}$with $\left|Y_{i}-\widetilde{Z}_{i}\right|<\sigma_{i} / 4$, and 4(ii): $B_{\sigma_{i / 4}}\left(Y_{i}\right) \cap S_{\alpha}^{+}=\varnothing$. In case 4(i) we can apply Theorem 1 to $\eta_{Z_{i}, 2 \sigma_{i}} M_{0}$ to give an embedded $C^{1}$ submanifold $L^{(i)}$ and balls $\left\{B_{\sigma_{i j}}\left(Y_{i j}\right)\right\}_{j=1,2, \ldots}$ such that, for some $\delta_{0}=\delta_{0}\left(\mathscr{M}, \mathbf{C}^{(0)}\right) \in\left(0, \frac{1}{16}\right)$,

$$
\begin{aligned}
& S_{\alpha}^{+} \cap B_{\sigma_{i} / 2}\left(Z_{i}\right) \backslash L^{(i)} \subset \bigcup_{j=1} B_{\sigma_{i j}}\left(Y_{i j}\right), \\
& \sum_{j} \sigma_{i j}^{m} \leq 2^{-m}\left(1-\delta_{0}\right) \sigma_{i}^{m}, \quad \mathscr{H}^{m}\left(L^{(i)}\right) \leq 2^{-m} \omega_{m} \sigma_{i}^{m} \leq \omega_{m} \sigma_{i}^{m} .
\end{aligned}
$$


Also, applying Lemma 2.6 to $\eta_{Z_{i}, 2 \sigma_{i}} M_{0}$ yields that $S_{\alpha}^{+} \cap \bar{B}_{\sigma_{i}}\left(Y_{i}\right)$ is contained in the $\delta(\varepsilon)$-neighborhood of $Z_{i}+q\left(\{0\} \times \mathbf{R}^{m}\right)$ with $\delta(\varepsilon) \downarrow 0$ as $\varepsilon \downarrow 0, \delta$ depending only on $\mathbf{C}^{(0)}$ and $\mathscr{M}$. Therefore, for $\varepsilon$ small enough (depending on $\mathrm{C}^{(0)}, \mathscr{M}$ only) we can cover $S_{\alpha}^{+} \cap \bar{B}_{\sigma_{i}}\left(Y_{i}\right) \backslash B_{\sigma_{i} / 2}\left(Z_{i}\right)$ by balls $\left\{B_{\tilde{\sigma}_{i j}}\left(\tilde{Y}_{i j}\right)\right\}$ such that

$$
\sum_{i} \tilde{\sigma}_{i j}^{m} \leq\left(1+2^{-m} \delta_{0}\right)\left(\sigma_{i}^{m}-2^{-m} \sigma_{i}^{m}\right)
$$

Thus, using this in combination with (5), we obtain a collection $\left\{B_{\sigma_{i j}}\left(Y_{i j}\right)\right\}$ satisfying

$$
\begin{aligned}
S_{\alpha}^{+} \cap B_{\sigma_{i}}\left(Y_{i}\right) \backslash L^{(i)} \subset \bigcup_{j} B_{\sigma_{i j}}\left(Y_{i j}\right) & \\
\sum_{j} \sigma_{i j}^{m} & \leq\left(\left(1-2^{-m}\right)\left(1+2^{-m} \delta_{0}\right)+2^{-m}\left(1-\delta_{0}\right)\right) \sigma_{i}^{m} \\
& =\left(1-2^{-2 m} \delta_{0}\right) \sigma_{i}^{m} \\
\mathscr{H}^{m}\left(L^{(i)}\right) & \leq \omega_{m} \sigma_{i}^{m}
\end{aligned}
$$

In case $4(\mathrm{ii})$ we can still use the fact that $S_{\alpha}^{+} \cap \bar{B}_{\sigma_{i}}\left(Y_{i}\right)$ is contained in the $\delta(\varepsilon)$-neighborhood of $Z_{i}+q(\{0\} \times \mathbf{R})^{m}$ and hence (since $\mathscr{H}^{m}\left(Z_{i}+q\left(\{0\} \times \mathbf{R}^{m}\right) \cap B_{\sigma_{i}}\left(Y_{i}\right)\right) \leq \omega_{m}\left(\frac{15}{16}\right)^{m / 2} \sigma_{i}^{m}$ in case 4(ii)), we can cover $S_{\alpha}^{+} \cap \bar{B}_{\sigma_{i}}\left(Y_{i}\right)$ by balls $B_{\sigma_{i j}}\left(Y_{i j}\right)$ such that (6) holds with $L^{(i)}=\varnothing$. Of course we can also trivially find a cover $B_{\sigma_{i j}}\left(Y_{i j}\right)$ such that (6) holds with $L^{(i)}=\varnothing$ in case $(4)^{\prime}$ holds. Thus we have shown that we can in all cases select a cover $\left\{B_{\sigma_{i j}}\left(Y_{i j}\right)\right\}$ for $S_{\alpha}^{+} \cap \bar{B}_{\sigma_{i}}\left(Y_{i}\right)$ such that (6) holds.

Repeating this process (starting with any one of the balls $B_{\sigma_{i j}}\left(Y_{i j}\right)$ in place of $B_{\sigma_{i}}\left(Y_{i}\right)$ and again using Theorem 1), we get embedded submanifolds $L^{i j}$ and balls $\left\{B_{\sigma_{i j q}}\left(Y_{i j q}\right)\right\}$ such that, with $\tilde{\delta}_{0}=2^{-2 m} \delta_{0}$,

$$
\begin{aligned}
& S_{\alpha}^{+} \cap B_{\sigma_{i j}}\left(Y_{i j}\right) \backslash L_{i j} \subset \bigcup_{q} B_{\sigma_{i j q}}\left(Y_{i j q}\right), \\
& \sum_{q} \sigma_{i j q}^{m} \leq\left(1-\tilde{\delta}_{0}\right) \sigma_{i j}^{m}, \quad \mathscr{H}^{m}\left(L_{i j}\right) \leq \omega_{m} \sigma_{i j}^{m} .
\end{aligned}
$$

Notice that by combining (6) and (7) we have 


$$
\begin{aligned}
& S_{\alpha}^{+} \cap B_{\sigma_{i}}\left(Y_{i}\right) \backslash\left(L^{(i)} \cup\left(\bigcup_{i} L_{i j}\right)\right) \subset \bigcup_{j, q} B_{\sigma_{i j q}}\left(Y_{i j q}\right) \\
& \sum_{q, j} \sigma_{i j q}^{m} \leq\left(1-\tilde{\delta}_{0}\right)^{2} \sigma_{i}^{m}, \quad \mathscr{H}^{m}\left(L^{(i)} \cup\left(\bigcup_{j} L_{i j}\right)\right) \leq \omega_{m} \sigma_{i}^{m}\left(1+\left(1-\tilde{\delta}_{0}\right)\right) .
\end{aligned}
$$

After repeating this argument $(N-2)$ times more we get a collection of balls $\left\{B_{\sigma_{N, i}}\left(Y_{N, i}\right)\right\}$ and $C^{1}$ submanifolds $L_{N, i}$ such that

$$
\begin{gathered}
S_{\alpha}^{+} \cap \bar{B}_{1} \backslash\left(\bigcup_{i} L_{N, i}\right) \subset \bigcup_{i} B_{\sigma_{N, i}}\left(Y_{N, i}\right), \quad \sum_{i} \sigma_{N, i}^{m} \leq\left(1-\tilde{\delta}_{0}\right)^{N} \sum_{i} \sigma_{i}^{m}, \\
\mathscr{H}^{m}\left(\bigcup_{i} L_{N, i}\right) \leq\left(\sum_{i} \omega_{m} \sigma_{i}^{m}\right)\left(1+\left(1-\tilde{\delta}_{0}\right)+\cdots+\left(1-\tilde{\delta}_{0}\right)^{N}\right) .
\end{gathered}
$$

Since $N$ is arbitrary this gives $\mu_{\sigma}\left(S_{\alpha}^{+} \cap \bar{B}_{1} \backslash \bigcup_{i, N} L_{N, i}\right)=0$ for each $\sigma \in$ $\left(0, \rho_{0}\right)$ so $\mathscr{H}^{m}\left(S_{\alpha}^{+} \cap \bar{B}_{1} \backslash \bigcup_{i, N} L_{N, i}\right)=0$, and $\mu_{\sigma}\left(S_{\alpha}^{+} \cap \bar{B}_{1}\right) \leq$ $\left(\mu_{\rho_{0}}\left(S_{\alpha}^{+} \cap \bar{B}_{1}\right)+1\right) \tilde{\delta}_{0}^{-1}$ for each $\sigma \in\left(0, \rho_{0}\right)$, so $\mathscr{H}^{m}\left(\mathscr{S}_{\alpha}^{+} \cap \bar{B}_{1}\right) \leq$ $\left(\mu_{\rho_{0}}\left(S_{\alpha}^{+} \cap \bar{B}_{1}\right)+1\right) \tilde{\delta}_{0}^{-1}<\infty$ as required. In view of the arbitrariness of $X_{0}$, this completes the proof.

6.2. Proof of Theorem 4. Without loss of generality we can assume that $X_{0}=0$. Let $\varepsilon>0$ be arbitrary for the moment, and pick $\rho$ small enough so that the cube $[-\rho, \rho]^{n+k}$ is contained in $U_{M}$ and

$$
\rho^{-n-2} \int_{M \cap[-\rho, \rho]^{n+k}} \operatorname{dist}^{2}(X, \mathbf{C})<\varepsilon^{2} .
$$

For small enough $\varepsilon,(1.17)$ guarantees that the slice $M_{z, \rho} \equiv\{(x, y) \in$ $\left.[-\rho / 2, \rho / 2]^{n+k} \cap M: y=z\right\}$ is not a smooth manifold for any $z \in$ $(-\rho / 2, \rho / 2)^{m}$. Then Sard's theorem yields that for $\mathscr{H}^{m}$-almost all $z \in$ $(-\rho / 2, \rho / 2)^{m}$ we must have

$$
S_{\rho} \equiv \operatorname{sing} M \cap\left\{(x, z): x \in(-\rho / 2, \rho / 2)^{l+k}\right\} \neq \varnothing .
$$

By (1.10), we know that $\mathscr{H}^{m}$-almost all $Z=(x, z) \in S_{\rho}$ are such that

$$
q\left(\mathbf{C}_{0} \times \mathbf{R}^{m}\right) \in \operatorname{Tan}_{Z} M
$$

for some cylinder $\mathbf{C}=q\left(\mathbf{C}_{0} \times \mathbf{R}^{m}\right) \in \mathscr{M}\left(q \in \mathrm{SO}\left(\mathbf{R}^{n+k}\right)\right)$. Also, since the set of densities $\Theta_{\mathbf{C}}(0)$ corresponding to such $\mathbf{C}$ is discrete by (2.1), 
from (1.16) and (1.5), it follows that $\rho>0$ can be chosen to ensure that $\Theta_{\mathbf{C}}(0)=\mu$ for all such cylinders $\mathbf{C}=q\left(\mathbf{C}_{0} \times \mathbf{R}^{m}\right)$, where $\mu$ is as in (1.16). Thus the alternative (i) of Lemma 1 always fails in this case, so by the same argument as in the proof of Theorem 1, there is $\sigma>0$ such that, if $\ddagger \ddagger$ holds for all $\mathbf{C}=\mathbf{C}_{0} \times \mathbf{R}^{m} \in \mathscr{M}$ with $\Theta_{\mathbf{C}}(0)=\mu$ and $\varepsilon$ is appropriately small, then

$$
S_{\rho}^{0} \cap(-\rho / 2, \rho / 2)^{n+k} \subset \operatorname{graph} w,
$$

for some embedded $C^{1, \mu}$ manifold of dimension $m$, where $S_{\rho}^{0}$ denotes the set of points $Z$ in $S_{\rho}$ such that (2) above holds, and $w \in$ $C^{1, \mu}\left((-\rho / 2, \rho / 2)^{m} ;\{0\} \times(-\rho / 2, \rho / 2)^{l+k}\right)$ satisfies

$$
|w|_{C^{1, \mu}} \leq C \varepsilon \text {. }
$$

However since there is at least one point of $S_{\rho}^{0} \cap\left\{(x, z) \in(-\rho / 2, \rho / 2)^{l+k}\right\}$ for $\mathscr{H}^{m}$-almost all $z \in(-\rho / 2, \rho / 2)^{m}$, it is evident that

$$
\overline{S_{\rho}^{0}} \cap(-\rho / 2, \rho / 2)^{n+k}=\operatorname{graph} w,
$$

and hence

$$
\operatorname{sing} M \cap(-\rho / 2, \rho / 2)^{n+k}=\operatorname{graph} w \cup K,
$$

for some set $K$ disjoint from graph $w$. Now we claim $B_{\sigma} \cap K=\varnothing$ for suitably small $\sigma>0$. Otherwise we can find a sequence $\left\{Z_{j}\right\} \subset K$ with $Z_{j} \rightarrow 0$. For sufficiently large $j$ we can choose $X_{j} \in \operatorname{graph} w$ with $\left|X_{j}-Z_{j}\right|=\min \left|X-Z_{j}\right|$ over all $X \in \operatorname{graph} w$. Since $\Theta_{M}(X) \geq$ $\mu$ for all $X$ in graph $w$ sufficiently close to 0 and since by (1.5) $\mu \leq$ $\left(\omega_{n} \sigma\right)^{-1} \mathscr{H}^{n}\left(M \cap B_{\sigma}\right) \leq \mu+\varepsilon(\sigma)$, where $\varepsilon(\sigma) \downarrow 0$ as $\sigma \downarrow 0$, and since $Z_{j}, X_{j} \rightarrow 0$, it follows from (1.5) that $\eta_{X_{j},\left|X_{j}-Z_{j}\right|} M \rightarrow \widetilde{M}$ with $\Theta_{\widetilde{M}}(0) \geq$ $\mu$ and $\lim _{\rho \uparrow \infty}\left(\omega_{n} \rho^{n}\right)^{-1} \mathscr{H}^{n}\left(\widetilde{M} \cap B_{\rho}\right)=\mu$. Thus by a well-known argument involving the monotonicity formula we deduce that $\widetilde{M}$ is actually a cone with vertex at 0 . On the other hand, by another application of (1.5) we have $\Theta_{\widetilde{M}}(X) \geq \mu$ (and hence is equal to $\mu$ ) at each point of $\{0\} \times \mathbf{R}^{m}$, and (see the discussion subsequent to (1.9), and $\left(1.9^{\prime}\right)$ ) we conclude that $\widetilde{M}$ is invariant under translations $X \mapsto X+(0, z), z \in \mathbf{R}^{m}$. But by construction there exists $Z \in \operatorname{sing} \widetilde{M} \backslash\{0\} \times \mathbf{R}^{m}$, and hence $\operatorname{dim} \operatorname{sing} \widetilde{M} \geq$ $m+1$, which contradicts the definition of $m$. Hence the proof of Theorem 4 is complete.

6.3. Proof of Corollary 3. Since $\Theta_{\mathbf{v}}\left(X_{0}\right)<2$ we can choose $\sigma_{0}>0$ such that $B_{\sigma_{0}}\left(X_{0}\right) \subset U$ and $\left(\omega_{n} \sigma_{0}^{n}\right)^{-1}\|\mathbf{V}\|\left(B_{\sigma_{0}}\left(X_{0}\right)\right)<2$. Notice that then 
$\mathbf{V}$ is multiplicity 1 ; in fact by $(1.5)$ there is $\sigma \in\left(0, \sigma_{0}\right)$ such that

$$
\sup _{Y \in B_{\sigma}\left(X_{0}\right), \rho \in(0, \sigma)} \frac{\|V\|\left(B_{\rho}(Y)\right)}{\omega_{n} \rho^{n}}<2-\varepsilon_{0} \text { for some } \varepsilon_{0}>0 .
$$

Let $\mathscr{V}_{0}$ be the class of all varifolds $q \eta_{Y, \rho \#}\left(\mathbf{V}\left\llcorner B_{\sigma}\left(X_{0}\right)\right)\right.$, where $Y \in$ $\mathbf{R}^{2+k}, \rho>0$, and $q \in \mathrm{SO}\left(\mathbf{R}^{2+k}\right)$. Also, given any open $W \subset \mathbf{R}^{n+k}$ we let $\mathscr{V}_{0}(W)$ be the set of all varifold limits, taken in $W$, of convergent sequences $\left(q_{j} \eta_{Y_{j}, \rho_{j} \#} \mathbf{V}\right)\llcorner W$ (thought of as varifolds in $W$ ) with $\bigcup_{j=1}^{\infty} \bigcap_{i=j}^{\infty} q_{j} \eta_{Y_{i}, \rho_{j}} B_{\sigma}\left(X_{0}\right) \supset W$, and let $\mathscr{V}=\bigcup_{W} \mathscr{V}_{0}(W)$. By virtue of the compactness and regularity theorems [1] the set $\mathscr{M}$ consisting of all the regular sets (in $W$ ) of all varifolds in $\mathscr{V}_{0}(W)$, taken over all open $W \subset \mathbf{R}^{n+k}$, is a multiplicity 1 class in the sense of $\S 1$, provided we take $U_{M}=W$ whenever $M=\operatorname{reg} \mathbf{T}$ for $\mathbf{T} \in \mathscr{V}_{0}(W)$. Furthermore by the analysis of [3] the only cylindrical cones $\mathbf{C}_{0} \times \mathbf{R}^{n-1}$ possible in this class are given by the cones $\mathbf{C}_{0}$ consisting of a union of three coplanar rays emanating from the origin in $\mathbf{R}^{k+1}$, and meeting at equal angles of $2 \pi / 3$. Notice also that $\mathbf{V}\left\llcorner B_{\sigma}\left(X_{0}\right) \in \mathscr{V}_{0}\left(B_{\sigma}\left(X_{0}\right)\right)\right.$. Now the corollary is proved by virtue of Theorem 4 and (1.10) in case $n \geq 3$.

To prove the additional claim in case $n=2$ we proceed as follows. Let $M=\operatorname{reg}\left(\mathrm{V}\left\llcorner B_{\sigma}\left(X_{0}\right)\right) \quad(\in \mathscr{M})\right.$. By virtue of (1.10) we have exactly two possibilities: either

(i) $\operatorname{Tan}_{X_{0}} M$ contains a cylindrical cone $q\left(\mathbf{C}_{0} \times \mathbf{R}\right)$ with $\mathbf{C}_{0}$ as described above, or

(ii) $X_{0} \in \mathscr{S}_{0}(M)$ so that no elements of $=\operatorname{Tan}_{X_{0}} M$ are cylindrical. In case (i) we can directly apply Corollary 2 in order to deduce that for some $\rho>0$, sing $M \cap B_{\rho}\left(X_{0}\right)$ is a properly embedded $C^{1, \alpha}$ Jordan arc with endpoints in $\partial B_{\rho}\left(X_{0}\right)$, so there is nothing further to prove in this case.

For case (ii) we first observe that each of the cones $\mathbf{C} \in \operatorname{Tan}_{X_{0}} M$ must have at least one ray of singular points. Indeed since $0 \in \operatorname{sing} C$ (by virtue of the regularity theorem [1] and the fact that $X_{0} \in \operatorname{sing} M$ ) otherwise C would have an isolated singularity at 0 . However then $\Sigma=\mathbf{C} \cap S^{k+1}$ would be a smooth embedded compact one-dimensional submanifold of $S^{k+1}$, and $\Sigma$ would have to be a finite union of a pairwise-disjoint great circles: $\Sigma=\bigcup_{j=1}^{N} \Sigma_{j}$, where each $\Sigma_{j}$ is a great circle. If $N=1$ this would give sing $C=\varnothing$, a contradiction. If $N \geq 2$, then $\boldsymbol{\Theta}_{M}\left(X_{0}\right)=\boldsymbol{\Theta}_{\mathbf{C}}(0) \geq 2$, again a contradiction. Hence each $\dot{C} \in \operatorname{sing} M$ has at least one singular ray as claimed. 
So, in case (ii), take $\mathrm{C} \in \operatorname{Tan}_{X_{0}} M$, and let $r_{1}, \cdots, r_{N}$ be the singular rays of $\mathbf{C}$. In view of the fact that along each of these rays $\mathbf{C}$ has normal cross section consisting of three coplanar rays meeting at angles of $2 \pi / 3$ (by the above discussion of cylindrical cones in $\mathscr{M}$ ), and in view of the fact that $\Theta_{\mathbf{C}}(Y) \leq 2-\varepsilon_{0}$ (by (1)), it is easy to check using (1.5) that there is a fixed constant $\theta>0$, depending only on $\mathscr{M}$ and $\varepsilon_{0}$, such that

$$
\operatorname{dist}\left(r_{i} \cap S^{k+1}, r_{j} \cap S^{k+1}\right) \geq \theta, \quad i \neq j,
$$

and in particular $N$ is bounded above by a fixed integer depending only on $\mathscr{M}$. Also by definition of $\operatorname{Tan}_{X_{0}} M$ there is a $\rho_{0}>0$ such that for each $\rho \in\left(0, \rho_{0}\right)$ there is a cone $\mathbf{C}_{\rho} \in \operatorname{Tan}_{X_{0}} M$ such that

$$
\rho^{-4} \int_{M \cap B_{\rho}\left(X_{0}\right)} \operatorname{dist}^{2}\left(X, \mathbf{C}_{\rho}\right)<\varepsilon^{2} .
$$

Equations (2) and (3) evidently imply that the number $N$ of singular rays $r_{j}$ in a cone $\mathrm{C} \in \operatorname{Tan}_{X_{0}} M$ is a constant. Furthermore if $Y \neq 0$ is on one of the singular rays $r_{j}$ of $\mathrm{C}$, then for suitable $\delta>0, \mathrm{C} \cap B_{\delta}(Y)$ is a union of three half-discs meeting along the common boundary $r_{j} \cap$ $B_{\delta}(Y)$ at angles of $2 \pi / 3$. Thus (cf. the argument at the beginning of $\S 6.2)$, for almost all $\rho \in\left(0, \rho_{0}\right), \partial B_{\rho} \cap M$ contains at least $N$ singular points $Z_{1}, \cdots, Z_{N}$, one in the $\varepsilon(\rho)$-neighborhood of each $r_{j} \cap \partial B_{\rho}, j=$ $1, \cdots, N$, where $\varepsilon(\rho) \downarrow 0$ as $\rho \downarrow 0$. Therefore by applying Theorem 4 to $\eta_{Y_{j, \rho}} M$ (and making using of the estimate (4) in $\S 6.2$ in the proof of Theorem 4), we deduce that there are exactly $N$ properly embedded locally $C^{1, \alpha}$ Jordan arcs $\Gamma_{1}, \cdots, \Gamma_{N}$, each with one endpoint in $\partial B_{\sigma}$ and with one endpoint at $X_{0}$, where $\sigma \in\left(0, \rho_{0}\right)$ is chosen suitably small, such that sing $M \cap \bar{B}_{\sigma}=\bigcup_{j=1}^{N} \Gamma_{j}$. The fact that the $\Gamma_{j}$ have finite length follows directly from the fact that (by estimate (4) of $\S 6.2) \Gamma_{j} \cap B_{\rho} \backslash B_{\rho / 2}$ has length $\leq \rho$ for sufficiently small $\rho$. (In fact (4) of $\S 6.2$ gives length $\Gamma_{j} \cap B_{\rho} \backslash B_{\rho / 2} \leq(1+\varepsilon(\rho)) \rho / 2$, where $\varepsilon(\rho) \downarrow 0$ as $\rho \downarrow 0$.)

\section{Concluding remarks}

The main discussion centered on the properties of the singular set. However, an examination of the relevant arguments will show that in fact we obtained various results about asymptotics of $M$ on approach to the singular set. For instance, the proof of Theorem 1 shows that, for given $\delta>0$, if we select $X \in \operatorname{sing}_{\alpha} M$ such that there exists $\sigma=\sigma(X, M)>0$ 
with

$$
\mathbf{B}_{\delta}\left(\Pi\left(B_{1} \cap \eta_{X, \rho} \operatorname{sing}_{\alpha} M\right)\right) \supset q_{\rho}\left(\{0\} \times \mathbf{R}^{m}\right) \cap B_{1}
$$

for all $\rho \in(0, \sigma)$, where $\Pi$ is the orthogonal projection of $\mathbf{R}^{n+k}$ onto an $m$-dimensional subspace $q_{\rho}\left(\{0\} \times \mathbf{R}^{m}\right), q_{p} \in \mathrm{SO}\left(\mathbf{R}^{n+k}\right)$, then, provided $\delta=\delta\left(\mathbf{C}^{(0)}, \mathscr{M}\right)$ is sufficiently small,

$$
\begin{aligned}
& \rho^{-n-2} \int_{M \cap B_{\rho}} \operatorname{dist}^{2}(X, \mathbf{C}) \\
& \leq C\left(\frac{\rho}{\sigma}\right)^{\mu} \sigma^{-n-2} \int_{M \cap B_{\sigma}} \operatorname{dist}^{2}(X, \mathbf{C}), \quad 0<\rho \leq \sigma,
\end{aligned}
$$

for some fixed cylindrical cone $\mathbf{C}=q\left(\mathbf{C}_{0}^{(0)} \times \mathbf{R}^{m}\right) \in \mathscr{M}, \mu=\mu(\mathscr{M}) \in$ $(0,1)$. That is, we have the following:

7.3. Theorem. Suppose $\alpha>1$ is given, and $\mathbf{C}^{(0)}=\mathbf{C}_{0}^{(0)} \times \mathbf{R}^{m} \in \mathscr{M}$ with $\boldsymbol{\Theta}_{\mathbf{C}^{(0)}}(0)=\alpha$ is such that $\ddagger \ddagger$ holds. If $M \in \mathscr{M}, X \in \operatorname{sing}_{\alpha} M$, and there is $\sigma>0$ such that (7.1) holds for all $\rho \in(0, \sigma)$, then (7.2) holds.

Remarks. (1) Notice that, for any given $\delta>0$, the hypothesis (7.1) is automatically satisfied (with $q_{\rho}$ independent of $\rho$ ) for some $\sigma>0$ at any point $X$ at which sing ${ }_{\alpha} M$ has an approximate $m$-dimension tangent plane $q\left(\{0\} \times \mathbf{R}^{M}\right)$ in the sense of [19]; since we have shown $\operatorname{sing}_{\alpha} M$ is countably $m$-rectifiable, we have (7.1) (hence (7.2)) at $\mathscr{H}^{m}$-a.e. $X \in$ sing $_{\alpha} M$.

(2) Notice that in particular this means that $\mathbf{C}$ is the unique tangent cone of $M$ at any such point $X$.

We also note here that if the hypotheses are as in Theorem 4 , then the proof of Theorem 4 shows that (7.2) holds uniformly for $X \in \operatorname{sing} M \cap$ $B_{\rho}\left(X_{0}\right)$ for suitable $\rho>0$, and it is standard that this implies there is a $C^{1, \alpha}$ diffeomorphism of $B_{\rho / 2}\left(X_{0}\right)$ onto itself which takes $\bar{M} \cap B_{\rho / 2}\left(X_{0}\right)$ onto $B_{\rho / 2} \cap \mathbf{C}^{(0)}$.

Finally we want to point out that all of the above extends in a straightforward manner to a Riemannian setting. Indeed in view of the Nash embedding theorem it is enough to consider classes $\mathscr{M}$ as in $\S 1$, except that in place of (1.1) we have

$$
\int_{M} \operatorname{div}_{M} \Phi=\int_{M} \Phi \cdot H_{M}
$$

for some function $H_{M}$ satisfying $\sup _{U_{M}}\left|H_{M}\right| \leq \Lambda_{M}$, where $\Lambda_{M}$ is a 
constant. Then in place of (1.5) we have that

$$
e^{-C \Lambda_{M} \rho}\left(\omega_{n} \rho^{n}\right)^{-1} \mathscr{H}^{n}\left(M \cap B_{\rho}(X)\right)
$$

is an increasing function of $X$ so long as $B_{\rho}(X) \subset U_{M}$, and the $L^{2}$ estimates of $\S 3$ generalize to this setting with "error term" $C \Lambda_{M}$ on the right (or with $C \Lambda_{M \rho}$ on the right in case of estimates taken over the ball $B_{\rho}$ rather than $\left.B_{1}\right)$. Then the proof of Lemma 1 easily generalizes to the case when $\left(1.1^{\prime}\right)$ is assumed with $\Lambda_{M_{j}} \leq \varepsilon_{j}$ in place of (1.1), provided we use $\beta_{j}=\left(\int_{M_{j} \cap B_{1}} d_{j}^{2}+C \Lambda_{M_{j}}\right)^{1 / 2}$ in place of $\left(\int_{M_{j} \cap B_{1}} d_{j}^{2}\right)^{1 / 2}$ in that argument. Then we can conclude that if the hypotheses are as in Lemma 1 , except that $\left(1.1^{\prime}\right)$ is assumed in place of (1.1), and if $\Lambda_{M}<\varepsilon_{0}$, then either alternative (i) of Lemma 1 holds, or there is $\widetilde{\mathbf{C}} \in \widetilde{\mathscr{C}}_{\gamma \varepsilon_{0}}\left(\mathbf{C}^{(0)}\right)$ such that

$$
\int_{M \cap B_{\theta}} \operatorname{dist}^{2}(X, \widetilde{\mathbf{C}}) \leq C \theta^{\alpha}\left(\int_{M \cap B_{1}} \operatorname{dist}^{2}(X, \mathbf{C})+\Lambda_{M}\right) .
$$

The reader should keep in mind that all elements of $\mathscr{C}_{\varepsilon}\left(\mathbf{C}^{(0)}\right)$ (and all elements of $\operatorname{Tan}_{X} M$ ) still satisfy (1.1), at least away from the singular axis $\{0\} \times \mathbf{R}^{m}$.

Of course once this modified version of Lemma 1 has been proved, then the proof of Theorem 1 carries over; the proof merely needs to be modified to allow additional terms like $C \sigma \Lambda_{M} 2^{-2 i}$ on the right side of inequalities like those in (3) and (4) of the proof of Theorem 1 . The reader can easily check that this makes no essential difference to the argument. Then the proofs of Theorems 2, 2' , 3, 4 and their corollaries carry over with the same proofs as before. Thus in conclusion we have:

7.4. Theorem. Theorems $2,2^{\prime}, 3,4$ are all valid in case the class $M$ consists of submanifolds $M$ satisfying $\left(1.1^{\prime}\right)$ in place of (1.1). In particular, mod 2 minimizing currents $T$ in any complete Riemannian manifold have interior singular sets as in Corollary 1 of $\S 1$.

\section{References}

[1] W. Allard, On the first variation of a varifold, Ann. of Math. (2) 95 (1972) 417-491.

[2] _ On the first variation of a varifold. Boundary behavior, Ann. of Math. (2) 101 (1975) 418-446.

[3] W. Allard \& F. Almgren, The structure of stationary one-dimensional varifolds with positive density, Invent. Math. 34 (1976) 83-97.

[4] __, On the radial behavior of minimal surfaces and the uniqueness of their tangent cones, Ann. of Math. (2) 113 (1981) 215-265. 
[5] F. Almgren, Existence and regularity almost everywhere of solutions to elliptic variational problems among surfaces of varying topological type and singularity structure, Ann. of Math. (2) 87 (1968) 321-391.

[6] __ Existence and regularity almost everywhere of solutions to elliptic variational problems with constraints, Mem. Amer. Math. Soc. 165 (1976).

[7] __, Q-valued functions minimizing Dirichlet's integral and the regularity of area minimizing rectifiable currents up to codimension two, preprint.

[8] E. De Giorgi, Frontiere orientate di misura minima, Sem. Mat. Scuola Norm. Sup. Pisa (1961) 1-56.

[9] H. Federer, Geometric measure theory, Springer, Berlin, 1969.

[10] __ The singular sets of area minimizing rectifiable currents with codimension one and of area minimizing flat chains modulo two with arbitrary codimension, Bull. Amer. Math. Soc. 76 (1970) 767-771.

[11] W. Fleming, Flat chains over a finite coefficient group, Trans. Amer. Math. Soc. 121 (1966) 160-186.

[12] D. Gilbarg \& N. Trudinger, Elliptic partial differential equations of second order, 2nd ed., Springer, Berlin, 1983.

[13] E. Giusti, Minimal surfaces and functions of bounded variation, Birkhäuser, Basel, 1984.

[14] F. Morgan, On the singular structure of two-dimensional area minimizing surfaces in $\mathbf{R}^{n}$, Math. Ann. 261 (1982) 101-110.

[15] R. E. Reifenberg, Solution of the Plateau problem for m-dimensional surfaces of varying topological type, Acta. Math. 104 (1960) 1-92.

[16] _ _ An epiperimetric inequality related to the analyticity of minimal surfaces, Ann. of Math. (2) 80 (1964) 1-21.

[17] R. Schoen \& L. Simon, Regularity of stable minimal hypersurfaces, Comm. Pure Appl. Math. 34 (1981) 741-797.

[18] L. Simon, Asymptotics for a class of non-linear evolution equations, with applications to geometric problems, Ann. of Math. (2) 118 (1983) 525-572.

[19] _ Lectures on geometric measure theory, Proc. Centre Math. Anal. Austral. Nat. Univ. 3 (1983).

[20] _ _ Entire solutions of the minimal surface equation, J. Differential Geometry 30 (1989) 643-688.

[21] __ On the singularities of harmonic maps, to appear.

[22] J. E. Taylor, Regularity of the singular sets of two dimensional area minimizing flat chains modulo 3 in $\mathbf{R}^{3}$, Invent. Math. 22 (1973) 119-139.

[23] _ , The structure of singularities in soap bubbles and soap-film-like minimal surfaces, Ann. of Math. (2) 103 (1976) 489-539.

[24] B. White, Regularity of the singular sets in immiscible fluid interfaces and solutions to other Plateau-type problems, Proc. Centre Math. Anal. Austral. Nat. Univ., Canberra 10 (1985) 244-249.

$[25]$ _

STANFORD UNIVERSITY 